Human Character in the IPIP: Towards Shorter, More Content-Valid, and Cross-Culturally Comparable IPIP-VIA Character Strength Scales

Matthias Bluemke

(GESIS - Leibniz Institute for the Social Sciences, Mannheim, Germany)

Melanie V. Partsch

(GESIS - Leibniz Institute for the Social Sciences, Mannheim, Germany)

Gerard Saucier

(University of Oregon, USA)

Clemens M. Lechner

(GESIS - Leibniz Institute for the Social Sciences, Mannheim, Germany)

Draft version: 1.01 (December 16, 2021)

This paper has not been peer-reviewed. 


\begin{abstract}
The "Values in Action" (VIA) framework is currently the most prominent approach to conceptualizing human character. VIA posits 24 character strengths that are purportedly valued across cultures and promote the well-being of both individuals and communities. However, unresolved limitations in the assessment of these character strengths continue to hamper theoretical progress in research on human character based on the VIA framework. Here we sought to lay a new foundation for advanced assessment of strengths by refining and extensively validating an existing open-science inventory from the International Personality Item Pool (IPIP). Using data from a U.S. community sample and four quota samples from the United Kingdom and Germany, we investigated whether valid, crossculturally comparable, and economical assessment of the VIA character strengths is possible with the IPIP. Experts selected suitable items with the aim to obtain 24 balanced-keyed short scales. Different experts then translated these items to German. Through exploratory and confirmatory factor analysis, we established essential unidimensionality and well-fitting measurement models for each scale. All scales achieved at least partial scalar invariance across languages. Reliability estimates were satisfactory. Extensive analyses of the strengths' nomological network placed character strengths between Big Five personality traits and basic human values, confirming that VIA strengths emphasize self-transcendence rather than self-enhancement. With few exceptions, the 24 character strengths scales were sufficiently distinct from the Big Five, and many showed incremental predictive validity, also for "good life" criteria. The 96-item inventory "IPIP-VIA-R" offers a sound and fully open-science approach to future research on character strengths.
\end{abstract}

Keywords: values in action (VIA), character strengths, big five traits, facets, human values 


\section{Human Character in the IPIP: Towards Shorter, More Content-Valid, and Cross-Culturally Comparable IPIP-VIA Character Strength Scales}

"The powers of man are finite, and if finite they are not too large for measurement." Sir Francis Galton (1884, The Measurement of Character, p.179)

\section{Theoretical Background}

Peterson and Seligman (2004) introduced the "values in action" (or VIA) framework and the accompanying "manual of sanities." Their intention was to broaden psychology's exclusive focus on psychopathology and undesirable human behavior (as expressed in the Diagnostic and Statistical Manual of Mental Disorders, e.g., American Psychiatric Association, 1994). With the aim to establish whether there are ubiquitous and universal virtues, and what exactly constitutes "good character", they reviewed the work of religious thinkers and (moral) philosophers from across the Eastern and the Western hemisphere and from ancient and modern times, for example, Plato's cardinal virtues and Aristotle's and Augustine's views building on them, and Aquinas's medieval theological virtues in line with Paul's ancient biblical writings. They also considered Eastern philosophy (Confucianism, Taoism). From this review ultimately emerged 24 universal, theoretically justified and empirically supported character strengths that feed into core virtues that recur throughout time and space. These character strengths are also reflected in the Values in Action Inventory of Strengths (VIA-IS) that intends to measure these 24 character strengths (Peterson et al., 2005). According to the VIA framework, these strengths can be mapped to six more abstract virtues (the "High Six" core virtues: justice, wisdom, temperance, courage, humanity, and transcendence), which themselves are too abstract to be measured directly and were theoretically, not factor-analytically derived (see Partsch et al., 2021). By comparison, the strengths are theoretically well-defined concepts and accompanied by prototypical exemplars (paragons), thus form the heart of measurement in the VIA framework.

In a multi-stage process, and inspired by psychological research on positive traits, Peterson and Seligman (2004) defined and classified 24 cross-culturally valued character strengths (see Table 1 for 
working definitions). They conceptualized character strengths as relatively stable, universally recognized and valued individual differences. Within the VIA classification, character strengths belonging to the same virtue have a common denominator, yet they are not replicas of one another (or necessarily highly correlated traits) but distinctive. High standing on one strength does not imply a similar standing on another strength mapped onto the same virtue. On the contrary, displaying all the strengths classified under a virtue is nearly impossible for individuals as strengths are different ways of expressing virtues.

To be included in the VIA classification, character strengths had to satisfy most of ten features: A distinctive and trait-like character strength should be fulfilling and contribute to a good life for oneself and others via manifold pathways. It must be (morally) valued in its own right, irrespective of its outcomes, and displaying a strength must elevate, not diminish or belittle other people. Therefore, society provides institutions and rituals associated with the cultivation of character strengths. A character strength implies that no desirable opposite trait exists (nonfelicitous opposite), and the absence of a strength must be observable in some individuals (selectivity). Cognitive abilities were excluded. Peterson and Seligman (2004) did not deem the VIA classification exhaustive; instead, they invited research that might lead to a revision (even expansion) of measures and their classification, though over the past 20 years the classification has not undergone revision, attesting to a rather consolidated and well-accepted framework (Park, 2018; Peterson \& Park, 2009; Ruch \& Proyer, 2015).

\section{Relevance of the VIA Character Framework}

Character strengths as defined by Peterson and Seligman (2004) relate traits to intrinsic motivations for behavior. The notion that character strengths are values in action highlights that these character strengths are value-laden traits. At the same time, the idea that they are values in action highlights that character strengths are skills that are expressed in behavior and, in principle, malleable and evolving over time. Skills need to be practiced by individuals across a variety of situations to become recognizable strengths and form signature strengths. Precisely such skillful mastery is likely to support individual well-being (Seligman, 2011). 
Character strengths reside at a similar level of abstraction as personality facets in the personality trait hierarchy. They form the building blocks of the VIA character trait space (McGrath et al., 2020; Partsch et al., 2021), resembling facets of traits in other trait spaces, such as the Big Five or HEXACO domains (Ashton \& Lee, 2007; McCrae \& John, 1992; Thalmayer \& Saucier, 2014). Yet, despite these similarities, differences between the character strengths and lexically derived personality traits are evident. Reminiscent of Allport's (1927) distinction between devaluated and evaluated personality, VIA includes purposively selected, cross-culturally morally valued character traits that are not sufficiently represented in (devaluated) lexical personality frameworks (e.g., Spirituality, Humor, Valor/Bravery, Social Intelligence; see Saucier, 2009). Instead of being lexically exhaustive, VIA aims to capture character traits that are morally valued and cross-culturally relevant.

Within personality science, the VIA character strengths offer a unique growth-oriented positive-psychological perspective that can complement other personality descriptors such as temperament, attitudes, values, beliefs, interests, or goals (Kandler et al., 2014). VIA's firm theoretical footing and its deliberate focus on value-laden and morally valued traits makes it a welcome addendum to lexical approaches in which moral and strongly evaluative person descriptors were eliminated (Peterson et al., 2005; Saucier \& lurino, 2020; Stahlmann \& Ruch, 2020). Moreover, the specific appeal of the VIA framework lies in its cross-cultural inspiration. Due to its broad trans-philosophical, cross-cultural and diachronic perspectives, the VIA framework is meant to be a universally applicable individual differences model (Dahlsgaard et al., 2005). It is therefore not surprising that the framework has received roughly 10,000 citations at the time of this writing (Ackerman et al., 2018).

\section{Criticism of Character Strength Assessment}

Ultimately the viability of the VIA framework as a conceptualization of human character and its utility for applied and research purposes hinges crucially on the extent to which the 24 proposed character strength can be validly assessed (DeYoung et al., 2007; Ones \& Viswesvaran, 1996). If scales do not reliably and validly measure what they purport to measure, then analyzing relations between character strengths and life outcomes, or group differences and intervention effects, is premature (e.g., Biswas-Diener, 2006; Park \& Peterson, 2010; Park et al., 2004; Seligman et al., 2005). However, 
despite VIA's important resource orientation and obvious relevance for clinical, educational, and workplace settings as well as its already widespread application in research and practice, the psychometric foundations of attendant measures lag far behind other individual differences constructs such as lexicon-based personality traits or values. Most of the positive-psychological literature is tellingly silent about the psychometric foundations of measuring character strengths (but rich in delving into philosophical perspectives and inspecting consequential outcomes). There are persisting limitations in the measurement of character strengths, including few validation attempts (Ackerman et al., 2018) that limit progress in the field and wider acceptance of character strengths in standard personality testing.

The authors of the VIA framework initially developed the 240-item VIA Inventory of Strengths based on self-report (VIA-IS for adults; Peterson et al., 2005; Peterson \& Seligman, 2004; VIA Institute on Character, 2020). In this research, we use public-domain IPIP-VIA items from the International Personality Item Pool (IPIP; Goldberg et al., 2006), which is based on a preliminary VIA-IS version provided by Peterson in June 2001 and described in Peterson and Seligman (2003) - a version that was then altered by Goldberg and Bel Bahar (https://ipip.ori.org/VIAkeys-explanation.htm). Peterson provided these items that mostly correspond to the original VIA-IS (which since then has been revised multiple times; its current version is available at https://www.viacharacter.org/researchers/assessments). Since then, several revised (e.g., VIA-IS-R), abbreviated (e.g., VIA-IS-120), and target-group specific versions (e.g., VIA-Youth) have been developed (McGrath, 2019). As only marginal differences have been found between the revised VIA-IS-R and its predecessor (VIA-IS), criticism is likely to pertain to both instrument families (see preprint by Vylobkova et al., 2021). There are also modified and alternative versions such as the Global Assessment of Character Strengths (McGrath, 2017), a 24vignettes based Character Strengths Rating Form (Ruch et al., 2014), and the structured interview focused on signature strengths (Peterson, 2003), but they are not at focus in this research.

The quality of available character strength measures has been repeatedly criticized. For example, Ng et al. (2017) found that more than half of all items from the VIA-IS needed to be excluded to achieve well-fitting measurement models for the 24 strengths (an iterative procedure merely based 
on low factor loadings). McGrath (2019) and McGrath and Wallace (2021) highlighted that many items lacked content validity and were too situation-specific to qualify as character (i.e., trait) measures. Even when good fit was found for measurement models for 24 strength scales (Feraco et al., 2021), it pertained to the truncated (Italian) version of the VIA-IS-120-a tool for which not only half of the items were dropped simply on the basis of maximizing item-total correlations, but that exclusively consists of positively-keyed items, and does not generalize to the predominantly used instruments. Besides these psychometric limitations, Noftle, Schnitker and Robins (2011) called for addressing construct validity of VIA scales by investigating links between strength scales and Big Five scales including their facets. Below, we discuss these issues with existing scales and raise several new ones.

Improper reliability estimates. During scale development, both Peterson et al. (2005) and Goldberg et al. (2006) focused mainly on achieving high levels of Cronbach's alpha coefficients for the scales (McGrath, 2019; Peterson \& Seligman, 2004; VIA Institute on Character, 2020). Unfortunately, alpha is a less-than-optimal criterion for scale development: It yields biased reliability estimates unless the scale satisfies a tau-equivalent measurement model (McNeish, 2018; Raykov, 1998, 2001), a requirement that these authors did not test and that neither the focal IPIP-VIA version nor the VIA-IS are likely to meet. Although applied research regularly analyzes composite scale scores for the 24 character strengths (e.g., McGrath, 2014, 2015, 2016), the reliability of such composites has not been established by fitting adequate latent measurement models. Hence, there is a need for refined strength scales that rest on proper measurement models and provide more trustworthy reliability estimates. Furthermore, estimates of test-retest reliability that provide more direct evidence of the replicability of scale scores are needed to complement internal consistency estimates.

Lack of unidimensionality. Unidimensionality refers to the quality of measuring exclusively a single construct, that is, the (trait) concept of interest (Hattie, 1985). Multidimensionality implies that more than one dimension underlies the items, rendering measurement of the construct complex. A realistic goal is essential unidimensionality, which reflects a dominant dimension while the conditional covariances over all item pairs, on average, must be small in magnitude (Stout, 1987). 
However, in practice, even "satisfactory" internal consistency coefficients are often misinterpreted as evidence for scale unidimensionality (Clark \& Watson, 2019; Cortina, 1993). Being silent about the requirements of unidimensionality of content-valid items is a questionable starting point for examining factor structures or analyzing reliability of character strength scales. Almost two decades after their inception, the dimensionality of available character strength scales (e.g., IPIP-VIA, VIA-IS, and later revisions such as VIA-IS-R; McGrath, 2019), especially closeness to unidimensional strength assessment, remain dubious.

Based on a review of 19 studies, Feraco and colleagues (2021) complained about the lack of evidence for unidimensional character assessment in the VIA-IS tradition, aside from the general paucity of testing proper measurement models for each character strength. Despite the lack of suitable measurement models in general, several major studies have already begun to address the higher-order structure of character strengths (e.g., McGrath, 2014, with $N>450,000$; see also Ng et al., 2017). However, when shedding light on the factorial validity of strength assessment, factoring whole item pools with multidimensional and interrelated strengths in the presence of overlapping items is unsuitable and likely to result in findings that do not replicate (see Feraco et al., 2021). A notable exception is Feraco and colleagues (2021) own study that comprehensively assessed the psychometric properties (i.e., unidimensionality, measurement models, hierarchical structure, and criterion validity) of the Italian version of the VIA-IS and found generally encouraging results for the short 120 -item version, despite remaining issues with some dimensions (e.g., Love of Learning). Yet there is not yet enough awareness that factor analyses of each strength scale are required as a first step to determine their dimensionality (see Ferrando \& Lorenzo-Seva, 2018). Only after the strength measurements have been optimized locally can their mutual relationships and hierarchical structures as well as their wider nomological net be validly studied (Boateng et al., 2018).

Lack of content validity and deficiencies in item wording. Content validity is arguably the most important property of a scale (Clark \& Watson, 2019). Maximizing internal consistency not only disregards content validity but can even conflict with it. A close look at some IPIP-VIA items highlights several issues with content validity that were likely present in VIA inventories from the initial 
development phase. First, quoting from IPIP-VIA, it is evident that not all items capture the definitional core of a character strength well despite high internal consistency. For example, "[I] have an imagination that stretches beyond that of my friends" (Originality) might not reflect useful creative imagination but escapism and daydreaming. Similarly, "[l] helped a neighbor in the last month" (Kindness) is probably too narrow and situation-specific.

Second, some items do not conform to the definition of character strengths as skills. Whereas some items do contain skill-related wording such as "being able to" or "being good at", many do not and instead represent mere attitudes (e.g., Citizenship/Teamwork: "[I] don't think it's important to socialize with others"; Equity: "[I] believe that everyone should have a say") [emphasis added].

Third, several items present content that is difficult to observe, ambiguous, vaguely quantified or idiomatic, such as "[I] read all the time" (Love of Learning), "[I] behave in unusual and strange ways" (Prudence), "[I] know what makes others tick" (Social Intelligence), "[I] prefer to participate fully rather than view life from the sidelines" (Zest), and "[I] don't approach things halfheartedly" (Zest). Ambiguous concepts, vague quantifiers, and idioms are either susceptible to subjective interpretations and range-restriction (Wänke, 2002), or difficult to translate and to adapt to other cultural contexts, thereby risking confusion among participants (Adelnia \& Vahid Dastjerdi, 2011; Liu, 2012). Interpretational ambiguity also occurs when items are not distinct but overlap with other strengths. For instance, "[I] refuse to take credit for work I have not done" (Equity) and "[I] take pride in not exaggerating who or what I am" (Integrity) convey Modesty in parallel. As others have noted before (e.g., Noftle et al., 2011), some items are not only hard to objectify but even difficult to evaluate subjectively, such as "[I] have never given bad advice to a friend" (Perspective) or "[I] am trusted to keep secrets" (Integrity), thereby inviting biased evaluations (John \& Robins, 1993; Vazire, 2010).

Finally, some scales provide imbalanced representations of the respective character strengths. For example, selecting Self-Regulation items with highly specific content, such as "[I] can stay on a diet" and "[I] can't resist eating candies or cookies if they are around, " creates a diet-specific facet in this measure and omits other domains in which Self-Regulation may manifest. Apart from this, the items presuppose that food is readily available and that holding a diet for health reasons is a 
widespread practice-a situation often found in economically developed (WEIRD) countries but less so in the Majority World.

Lack of cross-cultural applicability. The latter issue with Self-Regulation highlights another problem: Although Peterson and Seligman (2004) selected character strengths that they deemed cross-cultural universals, existing VIA scales use often use person-descriptors that are not applicable across cultures. Using items that refer to highly culture-specific concepts hampers cross-cultural comparability (Allalouf et al., 1999). For example, like the "diet" items cited above, the Spirituality/Religiousness item "[I] have spent at least 30 minutes in the last 24 hours in prayer or meditation" is likely to function differently across cultures where institutionalized prayers or meditation are common or not (e.g., Muslims' five daily prayers, or Salat). Similarly, "[I] go out of my way to attend educational events" (Love of Learning) will be understood differently in countries with free access to education compared to countries in which education strongly depends on socioeconomic status. Items that presuppose cultural codes and access to technology are likely prone to cultural bias, for example, "[I] consult the library or the Internet immediately if I want to know something" (Love of Learning). Character assessment that quantifies strengths and compares countries in terms of their scale scores implicitly subscribes to a generalizing ("etic" as opposed to culture-specific "emic") approach (Berry, 1969, 1999). However, we are unaware of any previous attempts at ensuring broad cross-cultural applicability of character strength items during item selection and testing measurement invariance of each strength scale across different languages, countries, or cultures. Before the equivalence of strength measurement has been firmly established, any statistical comparisons across countries are questionable (as in Biswas-Diener, 2006; Park et al., 2006).

Acquiescence. Acquiescence (i.e., the tendency to agree with survey questions irrespective of item content and keying, or "yeah-saying") is a widespread source of bias in survey research (Podsakoff et al., 2012). This bias is especially pressing in research involving samples that differ in their levels of acquiescent responding, as is regularly the case in cross-cultural research and in research involving respondents with varying levels of education and ability (for a review, see Lechner et al., 2019). If unaccounted for, acquiescence can introduce substantial bias in the means, factor 
structures, measurement invariance tests, and validity coefficients of personality and character strength scales (Lechner \& Rammstedt, 2015; McCrae et al., 2001; Primi et al., 2020; Rammstedt, \& Farmer, 2013; Soto \& John, 2017a, 2017b). Acquiescence is likely to diminish correlations between semantically opposite item-pairs and create a method factor among the reverse-keyed items (Cambré et al., 2002; Kam \& Meyer, 2015). Using balanced-keyed scales and modeling acquiescence as an additional factor is an established means of eliminating this bias in personality scales (Aichholzer, 2015; Billiet \& McClendon, 2000; Soto \& John, 2019). Current scale development practices clearly suggest that controlling acquiescence can largely eliminated the abovementioned biases. Thus, adequate measurement of character strengths requires balanced-keyed items that can eliminate acquiescent response bias. However, most empirical research to date has relied on unbalanced VIA-IS or IPIP-VIA character strength measures with only positively-keyed items or an unequal number of positively and negatively keyed items.

Scale length. Any 240 -item inventory entails substantial respondent burden that can impede the quality of measurement. When social surveys of the general population are intended, or largescale assessment takes place in international contexts, lengthy assessments are rarely feasible. Short forms alleviate respondent burden (see Rammstedt \& Beierlein, 2014; Schroeders et al., 2016; Ziegler et al., 2014) while often retaining much of their longer cousins' reliability and predictive validity (Rammstedt et al., 2020; Thalmayer et al., 2011). One challenge of short scales is retaining their validity via their breadth of content. The sweet spot found for Big Five scale length was around six items for broad domain scores (as in the BFI-2-S; Soto \& John, 2017a); for narrower facets, as few as two items can typically suffice (as in the BFI-2-S), although four items are advantageous (as is the case in the full BFI-2; Soto \& John, 2019). Combined with the goal of balancing each scale (acquiescence control), the challenges are obvious but-as the Big Five show-can be overcome.

\section{The Present Investigation}

\section{Rationale and Objectives}

Progress in the study of human character will require high-quality measures of character strengths that are comparable across languages and cultures. Both psychometric quality and cross- 
cultural validity must be considered early during scale development (Danner et al., 2016; Fischer \& Poortinga, 2018; Ziegler, 2014; Ziegler \& Bensch, 2013). To the extent that relatively little effort has been invested in constructing and validating scales that satisfy the requirements we discussed above (but see Stahlmann and Ruch, 2020, for a recent move towards scrutinizing strength measurement and its assumptions), the already large body of evidence on character strengths does not rest on a solid psychometric footing (e.g., Martínez-Martí \& Ruch, 2017). Only once such measures are available can one begin to answer fundamental theoretical questions about the stability of character traits, their relations to other individual difference constructs such as personality traits and values, or their predictive power for life outcomes.

Here we set out to advance the assessment of human character. Our aim was to establish whether it is possible to construct refined measures of the 24 VIA character that are (1) short scales (four items per strength, i.e., 96 items in total) and hence suitable for general population surveys, (2) content-valid, (3) cross-culturally applicable, and (4) balanced-keyed to control for acquiescent responding. Our additional self-imposed constraint was to (5) exclusively use existing items from the public domain, more specifically, those from the IPIP (Goldberg, 1999), which is freely available to researchers in line with open-science standards (Ashton, 2005).

The challenge was thus to achieve good psychometric properties while keeping scale length to a minimum. Short scales are sometimes criticized on the grounds that they may not be able to adequately represent a construct and achieve sufficient reliabilities (John \& Soto, 2007). However, these concerns are less pressing when-as in our present case-assessing narrow facets that can be well represented with a few items. Moreover, psychometric research generally allays many of the concerns about short scales. Decreasing the number of items under unidimensionality assumptions does not affect criterion validity, nor does it shift latent population means (Heene et al., 2014). Moreover, the super-brief BFI-10 can predict most outcomes as well as longer Big Five scales with only two items per Big Five dimension; the four-item BFI-2-XS performs slightly better and at a level comparable to much longer personality scales (Rammstedt et al., 2020; Thalmayer et al., 2011). A scale length of four items appears to be a good compromise between bandwidth and fidelity for narrow facets 
(e.g., Rammstedt et al., 2019; Soto \& John, 2017b; Thalmayer et al., 2011). Especially when using latent-variable models, the lower reliability of short scales does not impair predictive validity. Thus, the benefits of short scales in terms of enabling comprehensive assessments with minimal respondent burden arguably outweigh their disadvantages for our present intent, which was to create refined scales for research purposes (not individual diagnostics).

\section{Study Overview and Research Questions}

To revise and refine the IPIP-VIA character strength scales, we proceeded in five steps. First, we scrutinized the items of the 24 IPIP-VIA strength scales and reexamined known issues with unidimensionality and convergence of items in each strength scale on their respective common factor (i.e., their common variance proportion and factor loadings, based on a U.S. community sample). Second, experts evaluated the IPIP items for their closeness to the VIA concept definitions and against best practices for wording items. On this basis, aiming for a sweet spot between efficient and reliable measurement, we reduced the item set to four content-valid and balanced-keyed items per character strength. If necessary, we introduced alternate IPIP items from outside the IPIP-VIA item pool, mostly because some scales lacked suitable reversed items or needed improved wordings or better empirical psychometric properties (as determined in the next step). Third, we tested the preliminary scales with participants from the U.K. and-after a thorough translation and adaptation process-Germany. In these samples, we evaluated dimensionality, measurement model fit, and reliability. For a few scales, we developed alternative variants and compared the scale variants in two fresh samples from the U.K. and Germany. Fourth, we tested measurement invariance to ascertain crosscultural applicability of the 24 final scales (henceforth IPIP-VIA-R). Finally, we investigated scale validity. We cast a nomological net with Big Five personality traits (Costa \& McCrae, 1992; see Table 2a) and basic human values (Schwartz, 1992, 1994, 2006; see Table 2b). We also analyzed criterion validity for life satisfaction and health, next to gender differences in character strengths.

We organize our report according to the research questions that we sought to answer: RQ1. What is the dimensionality of each character strength in the IPIP-VIA item pool, and which items represent each character strength's core? RQ2. Does the IPIP comprise a sufficient number of content- 
valid items per strength such that a selection of cross-culturally applicable items would form balanced-keyed and (essentially) unidimensional short scales? RQ3. Can IPIP-VIA-R measurement models based on selection of four items per character strength achieve good fit? RQ4. Do brief balanced IPIP-VIA-R scales measure the character strengths reliably? RQ5. Are these IPIP-VIA-R short scales measurement invariant across two countries and languages? RQ6. Do the associations with relevant variables in the personality space support the construct validity of IPIP-VIA-R? RQ7. Do the IPIP-VIA-R scales possess criterion validity? Before we present the results for each $\mathrm{RQ}$, we give a general overview over the scale development and validation process.

\section{Overview Over the Scale Development and Validation Process}

This section comprises two parts. The first part explains the source material, the shortening and refinement of IPIP-VIA strength measurement, and the translation/adaptation process. Further details on the item selection process and scale validation are available from OSF at https://osf.io/3mfyw/?view only=529e048441414faa9b39a3ed6880123c). The second part describes samples, sample size considerations, and study material.

\section{Source Questionnaire for Self-Reported Character Strength Items: IPIP-VIA}

The IPIP (Goldberg, 1999; Goldberg et al., 2006) is a large open-science repository of more than 3,200 personality questionnaire items (https://ipip.ori.org/HistoryOfTheIPIP.htm). IPIP scales are similar to widely used commercial personality inventories, yet they are placed in the public domain and hence free to use for all purposes without licensing charges. Peterson and Seligman's (2004) "Values in Action" (VIA) approach to surveying character features among the most widely used "human character" inventories. Around the year 2000, the parallel IPIP-VIA scales comprised 240 items, with 10 items per character strength, except for Valor/Bravery (11 items) and Hope/Optimism (9 items). These items are largely identical to a preliminary version of the "VIA Inventory of Strengths" (VIA-IS) and were contributed by Peterson and subsequently adapted by Goldberg (1999) to fit the style and response format of the IPIP (see https://ipip.ori.org/Finding Scales to Measure Particular Constructs.htm). In 2005, a slightly revised set of IPIP-VIA strength scales was developed (Diamond et al., 2010; Goldberg et al., 2006). With the aim to develop shorter, more internally 
consistent scales, around $10 \%$ of the items were dropped or reworded, and a few items were replaced by new items that were not in the initial VIA item pool. According to the IPIP website, IPIP-VIA now comprises 252 items, but the website suggests omitting 39 items to optimize Cronbach's Alpha (which, we argue, is a less-than-optimal criterion for scale development), leaving 213 recommended items at present. The resulting IPIP-VIA scales are not balanced-keyed, and the proportion of reversekeyed items varies across scales, making it impossible to adequately control for acquiescence.

\section{Revising and Refining IPIP-VIA Strength Measurement for Cross-Cultural Use}

Reducing the initial IPIP-VIA item set. Starting from the 252 IPIP-VIA items (https://ipip.ori. org/newVIAKey.htm), we set out to abbreviate the 24 strength scales by filtering out unsuitable items. Four independent expert raters inspected the items in order to (1) identify the four best (i.e., most content-valid and best-worded items) per strength while (2) avoiding redundancy within each scale and reducing overlap with other scales and (3) eliminating items that seemed prone to cultural bias or other cognitive errors in the response process. Another constraint was to ideally (4) retain the same number of positively and negatively worded items per scale. We found that the 252 IPIP-VIA items were insufficient to arrive at 24 content-valid and balanced-keyed strength scales (see RQ1).

Adding additional IPIP candidate items. To balance the scale with regard to content-valid and reverse-keyed items, we broadened the item pool by adding items from other IPIP sections (see SOM_IPIP-VIA-R_Method_Item-Source-History.xIsx). We screened 3193 (non-VIA) items (variable sets termed $A-Y),{ }^{1}$ of which 126 were potentially suitable for measuring VIA character strengths. Out of these, we chose seven items that matched the strength definitions, were linguistically sound,

\footnotetext{
${ }^{1}$ We based our revision on standard IPIP items, but not newly added (Rasch-modeled) VIA items that were exclusively used in a South African context (variable set W; du Plessis \& de Bruin, 2015). Concerns about low sample size, the specific study context (as well as unavailability to an extant U.S. sample), and content validity cast doubts on their utility and the respective Rasch analyses.
} 
translatable, and free from cultural bias. We resolved lack of consensus in the item selection by comparing two questionnaire versions (Versions A and B; see SOM_IPIP-VIA-R_Method_Item-Selection.xlsx). In Version B, five items from five strength scales differed from Version A; additionally, five items from four strength scales obtained an improved translation (see RQ2).

Item selection to form ultimate IPIP-VIA-R questionnaire. We based the final decisions about which items from Versions $A$ and $B$ to include in the final 96-item IPIP-VIA-R on empirical results regarding dimensionality, model fit, and reliability. Among the decision criteria were factor loadings and goodness-of-model fit indices for acquiescence-controlled measurement models, closeness to unidimensionality, several reliability estimates, but also considerations of content validity and quality of translated items (see RQs 1-4).

\section{TRAPD Approach: Item Translation and Adaptation}

To ensure the cross-cultural applicability of the IPIP-VIA-R from the start, we jointly performed the item selection in samples from two cultures and languages: English (USA and U.K.) and German (Germany). Because the IPIP-VIA items were not available in German, we adapted the English items for the German-speaking context. We followed Harkness's (2003) Translation, Review, Adjudication, Pretest, and Documentation (TRAPD) approach, which is the current gold standard translation approach. We also adhered to best-practice guidelines for translation and adaptation (Behr \& Zabal, 2020). Three personality experts (native speakers of German and well-versed in English) independently prepared parallel translations. The translators plus two reviewers (native German survey methodologists with a strong command of English) agreed on the preferred item translations. An expert in cross-cultural studies and translation acted as an adjudicator during the review meeting and assessed the final versions vis-à-vis the source items. We documented all steps and decisions in the translation process (see SOM_IPIP-VIA-R_Method_TRAPD-Approach.xlsx and the accompanying documentation SOM_IPIP-VIA-R_Method_TRAPD-Approach.pdf).

During the adaptation process, we slightly improved translations compared to their English source versions where necessary, such as by eliminating minor inaccuracies in concept representation or by reducing overlap with neighboring scales through proper wording. We retrospectively 
coded any item alterations (from level 0 to level 4), with $0=$ translating straightforwardly; $1=$ addressing linguistic complexities; 2 = providing cultural adaptations (beyond linguistic issues); $3=$ overcoming measurement-related issues (in the source item); 4 = aligning content more closely with the construct definition (potential misrepresentation of construct in the source). Table 3 provides an overview of the alterations (counting across the two variants we tested). These changes arguably brought the German adaptation closer to the construct definitions of the VIA character strengths, such that the German, rather than the English IPIP items, might be best suited to serve as a source for future translations.

For the response scales, we kept the IPIP's default of five response categories but slightly adjusted the English response labels [and subsequently in German] to strengthen the cross-cultural applicability of the response scale: 1 = Does not apply at all [Trifft gar nicht zu], 2 = Applies only slightly [Trifft eher nicht zu], $3=$ Applies moderately [Teils, teils], $4=$ Applies mostly [Trifft eher zu], $5=$ Applies completely [Trifft voll und ganz zu].

\section{Samples and Data Quality}

We used five samples from three nations during three stages of the item selection and scale validation process: In the first stage, we used an extant U.S. community-dwelling sample $(98.4 \%$ White non-Hispanic or Euro-American, reported as "Caucasian", which reflects the ethnic composition of homeowners around 1993, that is, at the time of creating the panel). In the second stage, we collected two samples of online panelists who were recruited simultaneously in the U.K. and Germany via commercial online access panels. In the third stage, we used two additional independent online-samples from the U.K. and Germany. We obtained respondents' informed consent by agreement to the panel provider's study invitation (or by signature to the original data collectors in the United States). We chose sample sizes of around 500 to ensure sufficient ( 90\%) statistical power for testing the intended measurement models (see SOM_IPIP-VIA-R_RQ3_Sample-Size_Power-Analysis.pdf). Online samples were anonymously collected in two waves without tracking participants' ethnicity. (Mirroring their society, most of them were likely White, too, though specific minority proportions remain unknown.) Table 4 shows the sample compositions. 
Sample 1. The Oregon Research Institute (ORI) recruited a sample of approximately 750 U.S. homeowners in the Eugene-Springfield (Oregon) community who have completed IPIP items and a large set of other psychological measures. These measures were collected in multiple survey waves that encompass several years. (Not all Sample 1 respondents completed all sections though). We used extant data from this "Eugene-Springfield Community Sample" (ESCS; Goldberg \& Saucier, 2016) for initial scale inspection and shortening. Participants had worked on a paper-pencil interview on “Perceptions of Personal Qualities" (PPQ) in 2004 in exchange for a usual remuneration (roughly \$15 at the time). Data from 713 participants on 252 IPIP-VIA items were available (711 complete cases for factor analyses, because two participants had accidentally skipped 29 out of $342 \mathrm{PPQ}$-items items en bloc, and one of them produced another isolated missing value). A varying case number (with up to 126 missing cases) resulted when analyzing the non-VIA IPIP items added retroactively to the present-day IPIP-VIA item set.

Samples 2 and 3. In late 2018, a total of 1,021 native speakers from the U.K. and Germany completed the online survey, which allowed a glance at the item selection including non-VIA items that were taken, for the first time, by all study participants and at the same time. The quota sample was drawn by a commercial online access panel provider (respondi AG) in exchange for a small monetary compensation (roughly $2 €$ ). The sample matched the register-based German census proportions cross-classifying gender, three cohorts (age 18-29, 30-49, 50-69) and three educational levels attained (basic, middle, high education; Gauckler \& Körner, 2011). For comparable subsamples, respondi drew a matched quota sample in the U.K. Completion rates were lower in the U.K. (27.11\%) than in Germany (49.85\%), hinting at higher motivation and potentially higher data quality among the German panelists. After filtering out respondents with poor quality data, the analytical samples comprised 921 respondents (455 from the U.K., 466 from Germany).

Samples $\mathbf{4}$ and 5. For finalizing the item selection and validating measurement models across contexts, we recruited two additional samples (total $N=1,040$ ) via respondi in 2019 , none of whom had participated in the earlier wave. Again, there were differences in completion rates between the 
U.K. (33.14\%) and Germany (53.68\%). Filtering out low quality data, the analytical samples comprised 950 respondents ( 474 from the U.K, 476 from Germany).

Quality control (filtering). For the secondary data analysis of sample 1 (paper-and-pencil questionnaire), we kept all responses of the existing data set. For samples 2 through 5 (online surveys), we followed suggestions to filter out careless responders in line with Meade and Craig (2012). In the absence of scientific consensus for a standard procedure (Leiner, 2019), we excluded participants who did not pass minimum quality thresholds but not more than $10 \%$ of each sample, by combining three non-reactive indicators²: (1) To filter out low internal motivation (rushing/patterning), we kept only those panelists who spent more than an average second per relevant personality item according to server-side recorded time-stamps for each questionnaire page. (2) We computed each respondent's ipsative variance across all their ratings on strength and other personality items. We dropped $5 \%$ of participants in the lower tail of each country's score distribution, as low ipsative variance indicates straight-lining or non-differentiation. (3) Across the same items, we computed a robust multivariate Mahalanobis distance, $D$, that can handle missing data (Béguin \& Hullinger, 2004). $D$ quantifies the distance of an individual's response vector from the average response vector and indicates deviant response patterns. A high distance indicates highly unusual response patterns, such as due to inattentive responding or faking. We removed per country $2.5 \%$ of participants with $D$-values exceeding the 97.5\%-quantile. For details on the quality-enhancing procedure, see SOM_IPIP-VIA-R_ Method_Data-Quality.pdf.

${ }^{2}$ We identified careless responders indirectly, yet we mention here four interspersed direct attention checks and two diligence items in the questionnaire (as part of an unrelated project on careless responding). 


\section{Material}

All surveys involved multiple item-batteries, questions, and constructs unrelated to the current study, which we will not cover here. For details on specific validation criteria (e.g., Big Five and Schwartz Human Values), we refer the reader to RQ6 and RQ7.

IPIP-VIA questionnaire for Sample 1. In Fall 2004, as part of the PPQ-study with 342 personality items, the ESCS-participants were also administered IPIP-VIA items. We re-analyzed all 252 IPIPVIA items. Recall that the IPIP-VIA webpage presently recommends 213 IPIP-VIA items that overlap with the original VIA-IS items (Goldberg \& Saucier, 2016). ${ }^{3}$

IPIP-VIA-R (Version A) for Samples 2 and 3. Participants worked on 96 items for IPIP-VIA-R short scales (Version A) that we distributed across ten pages of a longer 200 -item online survey. In both countries, the IPIP-VIA-R section took about $9 \mathrm{~min}$. to complete. Rater disagreement about item selection and our initial empirical evaluation of Version A necessitated a crosscheck of scales with five alternative items and another five new translations of original items (Version B).

IPIP-VIA-R (Version B) for Samples 4 and 5. Because the IPIP-VIA-R items were presented towards the end of a longer survey in Samples 2 and 3, fatigue might have impaired data quality. Therefore, in Samples 4 and 5 that we used as the ultimate basis for the psychometric validation of IPIPVIA-R, we presented Version B of IPIP-VIA-R as the first substantive part following a short block of generic questions (e.g., socio-demographic questions). To allow for a comparison between Versions $\mathrm{A}$ and $B$, we also included the items from Version $A$ immediately after the 96 Version $B$ items (U.K.: five original items; Germany: five original items plus five original translations). The B-versions replaced

${ }^{3}$ Some scoring keys on the IPIP webpage (https://ipip.ori.org/newVIAKey.htm), which served as the inspirational starting point for our investigation, differ slightly from the scoring keys used on the ESCS data presented on the Harvard Dataverse archive (https://dataverse.harvard.edu/dataverse/ESCS-Data). A description of how those differences arose can be found at https://ipip.ori.org/VIAkeys-explanation.htm. 
their precursors at the respective questionnaire positions of Version A. Working on 101 (106 German) items lasted $10 \mathrm{~min}$. (12 min.) with an average completion time of about $20 \mathrm{~min}$. for the survey.

Data preparation and statistical analysis. We performed the preparatory steps and analyses with open- and closed-source software. We ran descriptive analyses and unconstrained factor analyses in SPSS (version 24.0) and FACTOR (version 10.8.04; Lorenzo-Seva \& Ferrando, 2006, 2013), confirmatory factor analyses in Mplus (version 7.31; L. K. Muthén \& B. O. Muthén, 2012) with the $R$ package MplusAutomation (version 0.7-3; Hallquist \& Wiley, 2018) as well as with the $R$-package lavaan (version 0.6-5; Rosseel, 2012) in R 3.4.4 (R Core Team, 2018). We gained additional information from the $R$-package $p s y c h$ (version 1.8.12; Revelle, 2019). Validity correlations were computed in Stata (version 15) and $R$.

\section{Research Question 1:}

What is the dimensionality of each character strength in the IPIP-VIA item pool, and which items represent each character strength's core?

Items should reflect the relevant target dimension (i.e., the common core of the construct). Yet, when factor-analyzing all VIA-IS strength items jointly (McGrath, 2014), many items show only small loadings on the assigned strength factor $(\lambda<.40)$, indicating rather loose item-factor relationships (Hair et al., 2006). Given the statistical evidence and conceptual criticism of other VIA character strength inventories (see also above), we tested whether-as we assumed-the same issues also plague the 252 IPIP-VIA items. Expecting confirming evidence in this regard, we sought to statistically identify suitable candidate items at the same time. We explored scale dimensionality and factor loadings for each IPIP-VIA strength scale through exploratory factor analyses (EFA) separately for each strength (Ferrando \& Lorenzo-Seva, 2018; Saucier \& Goldberg, 1996). Later, we tested measurement models for short scales via confirmatory factor analyses (CFA) with fresh samples (i.e., Samples 2-5).

Factor analysis casts light on the dimensionality of an item set, and unidimensionality is a highly desirable measurement property particularly for short scales (Bond \& Fox, 2015; Hattie, 1985). Lack of unidimensionality complicates or even invalidates the common practice of summating across ratings and interpreting the test scores as if they reflect a single construct. Measures of internal 
consistency such as Cronbach's Alpha also require (but do not test) unidimensionality and are biased if unidimensionality does not hold (McNeish, 2018; Raykov, 2001). Strict unidimensionality requires the absence of secondary factors (EFA) or correlated residuals (in CFA), which may be rooted in identical keywords or grammatical constructions. However, personality items are often factorially complex and rarely behave as markers of a single factor (Cattell, 1986), especially if items and scales are not thoroughly constructed. Essential unidimensionality is then a more realistic goal (Stout, 1987). It entails that the common factor explains most variability in scores, while additional factors (e.g., method factors) explain only small variance portions (or item-pair covariances) that do not harm measurement, at least when properly specified in a measurement model. Attaining (essential) unidimensionality often requires item selection and/or model re-specification (Brown, 2015; Gerbing \& Anderson, 1988; Hopwood \& Donnellan, 2010).

To gauge the extent to which issues hamper the assessment of character strengths with IPIPVIA scales-and to identify positively and negatively keyed candidate items for balancing the scales - we analyzed each of the 24 character strengths as measured with all available 252 IPIP-VIA items. For this purpose, we ran a secondary data analysis on Sample 1.

Previous issues about dimensionality demonstrated almost exclusively for VIA-IS character strength measures are likely to re-emerge with IPIP-VIA scales because (1) several IPIP-VIA items show poor wording or low content validity already at the surface level, (2) content similarities between subsets of items within character strength scales suggest unintended item clusters or "subfacets", and (3) despite some negatively keyed items, acquiescent responding likely introduces a secondary factor (method variance). Negatively worded items typically form a method factor (Bagozzi, 1993; Brown, 2015; Marsh \& Grayson, 1995) which, if unmodeled, is a potential source of model misfit that also blurs associations with external criteria (Rammstedt \& Farmer, 2013; Soto \& John, 2019). Given their common starting point in history, IPIP-VIA weaknesses are likely inherited by VIA-IS and later revisions. 


\section{Method}

In a first step, we examined descriptive statistics for each item (details in SOM_IPIP-VIAR_RQ1_Descriptives.pdf). The skew of the variables rarely exceeded an absolute value of +2 , while absolute excess kurtosis values of +3 occurred (where zero can be expected for normal distributions). Yet, Bartlett's tests of sphericity and sampling adequacy according to the Kaiser-Meyer-Olkin criterion (KMO = .78-.92; Dziuban \& Shirkey, 1974; Kaiser \& Rice, 1974) supported the suitability of the 24 scales for factor analysis (see Table 5).

We judged unidimensionality by (1) parallel analysis (Horn, 1965), which compares Eigenvalues obtained from principal axis factoring (PAF) and principal component analysis (PCA) against the $95^{\text {th }}$ percentiles of Eigenvalues resulting from 500 draws of identically structured random data; (2) the minimum average partial (MAP) test (Velicer et al., 2000); (3) factor loadings on the common single factor, indicating the quality of unidimensional measurement; (4) item communality when extracting multiple factors (with initial Eigenvalues $>1$ ) after applying orthogonal Varimax rotation (the most parsimonious explanation of item correlations by independent factors) as well as the accompanying proportions of variance captured by the $k$ extracted factors (indicating of the size of potentially relevant secondary factors). ${ }^{4}$

\section{Results}

Visual inspection of Eigenvalues (PAF scree plots) revealed that most scales had a strong first factor, after which Eigenvalues leveled off (see Figure 1). The first factors explained between $26 \%$ (Self-Regulation) and 56\% (Spirituality/Religiousness) of the variance in each strength scale (see

${ }^{4}$ We do not assume that orthogonality reflects "true" factor structures underlying strengths. We merely used Varimax as a tool to establish the necessary minimum number of factors and their associated variance proportions. These values merely served descriptive statistical purposes (not substantive interpretation). With default settings, SPSS provides no details on factors with initial Eigenvalues $<1$ that are not of interest here. 
SOM_IPIP-VIA-R_RQ1_Dimensionality.pdf). At the same time, parallel analysis suggested that more than three dimensions were present in 18 of the scales, and a secondary dimension was likely present in the remaining 6 scales. For instance, whereas a single dimension seemed sufficient for Originality, Spirituality, Humor, and Prudence according to the MAP-test, PAF parallel analysis suggested two, three, four, and five common factors to underlie the respective strengths. Although MAP often suggested fewer dimensions than parallel analysis (see Table 6), it should be noted that underfactoring (specifying too few factors) is considered a more severe error than overfactoring (specifying too many factors; Fabrigar et al., 1999). Also note that acquiescence can bias the number of factors to retain upward in classical random parallel analysis, but downward in the MAP-test and permutation parallel analysis (Valentini, 2017). The variance attributed to a second or third factor was often nonnegligible (e.g., after rotation two factors equally contributed $15 \%$ of common variance to Forgiveness; Appreciation of Beauty and Excellence comprised three factors with common variances of $16 \%, 15 \%$, and $11 \%)$.

Extracting a single-factor per strength in EFA, most loadings were of modest size (see SOM_ IPIP-VIA-R_RQ1_Dimensionality.pdf). Twenty items loaded lower than $\lambda<.25$, and some loadings were low enough to disqualify the attendant items as indicators of the common strength factor (e.g., "Have never given bad advice to a friend" obtained only a standardized loading of $\lambda=.10$ on the Perspective factor). Even when disregarding the 39 out of 252 IPIP-VIA items not recommended on the IPIP webpage (to improve Cronbach's alpha), 28 of the 213 (i.e., more than 10\%) currently used IPIPVIA items had loadings below $\lambda=.40$, indicating rather loose item-factor relationships (Hair et al., 2006). Our findings coincide with McGrath's (2014), who showed that also in the VIA-IS, a non-negligible number of items loaded $<.40$ on the strengths they were supposed to measure in a joint itemfactor analysis combining all the VIA-IS strength items. The loadings already imply some item heterogeneity in the strength scales and somewhat weak item-total correlations for several items.

The few reverse-keyed items provided by IPIP-VIA made it unlikely to arrive at high-quality balanced-keyed strength scales. Not only that scales comprised few reversed items per se, but the items also often insufficiently captured the strength core, as reflected in poor standardized loadings. For 
example, of three negative Forgiveness items "[I] hold grudges" works well $(\lambda=-.71)$, but the loadings for "[I] am unwilling to accept apologies" $(\lambda=-.25)$ and "[I] do not give anyone a second chance to hurt me" $(\lambda=-.31)$ lay a shadow over our ability to arrive at balanced-keyed scales, even when opting for merely four IPIP-VIA items per strength.

Extracting between two and five factors with EFA (depending on the Eigenvalues for each strength) made it clear why this was the case. Several items had low communalities, suggesting that these items fall outside the trait space of their targeted strength. For instance, researchers are discouraged on the IPIP-VIA webpage to use the item " $[I]$ could never stop loving my family and close friends, no matter what they did" for measuring Capacity for Love, a recommendation we confirmed through a disappointingly low communality $\left(h^{2}=.06\right)$. However, similar weaknesses existed in items that are recommended for strength measurement with IPIP-VIA, such as "[I] admit when I am wrong" (Equity, $h^{2}=.12$ ) and "[I] am not confident that my way of doing things will work out for the best" (Hope, $\left.h^{2}=.15\right)$, though other recommended items fared comparatively better $\left(h^{2}=.25-.56\right)$. At the same time, some items are not recommended for strength measurement with IPIP-VIA despite reaching similar and reasonable levels of communality (e.g., [I] am rarely aware of the natural beauty in the environment", Appreciation of Beauty and Excellence, $\left.h^{2}=.25\right) .^{5}$

\section{Discussion}

To conclude, many IPIP-VIA items currently recommended on the IPIP website appear problematic from a purely statistical (factor-analytic) standpoint already-other issues such as content validity and cross-cultural applicability aside. The assumption of unidimensionality is questionable for

\footnotetext{
${ }^{5}$ Two Heywood-cases (i.e., an item communality reached or exceeded $100 \%$, or a standardized loading
} exceed 1.00) occurred. Extracting the number of factors suggested by parallel analysis may be statistically necessary for two scales (CAP, FOR), but does not yield factors that are sufficiently "overdetermined" by multiple variables (i.e., single items may determine their last factors). 
all IPIP-VIA strengths (Table 6). On the basis of findings of parallel analysis, even essential unidimensionality seems out of reach for most of the scales. Although not all additional factors indicated by parallel analysis may reflect substantive dimensions, the deviations from unidimensionality were severe enough to prevent several items from loading substantially on a common strength factor. Moreover, several of the 252 items from the IPIP-VIA item pool had low communalities in multi-factor models, including several items in the currently recommended 213-Item IPIP-VIA scales. Our findings question the item quality, scale compositions, and the appropriateness of using IPIP-VIA scale sums or scale means. While they supported our approach towards shortening the IPIP-VIA scales, these findings also limited our options for balancing the scales. To allow for balanced, content-valid and (essentially) unidimensional character strength measures, the IPIP-VIA scales needed to be refined.

\section{Research Question 2:}

Does the IPIP comprise a sufficient number of content-valid items per strength such that a selection of cross-culturally applicable items would form balanced-keyed and (essentially) unidimensional scales?

The violations of unidimensionality identified in RQ1 call for a careful item selection to weed out problematic items. Yet, purely quantitative approaches to item selection (e.g., optimizing internal consistency) do not automatically ensure content validity: "If the content of an instrument cannot be defended with respect to the use of the instrument, construct validity cannot be obtained" (Sireci, 1998, p. 112). Five conditions must be satisfied to ensure content validity of a measure (Guion, 1977): The content domain is relevant to the purposes of measurement; the domain is unambiguously defined; the behavioral content has an accepted meaning or definition; qualified judges consensually agree that the domain has been adequately sampled; and the content must be observable and evaluated. Moreover, content validity in one culture or language does not automatically ensure cross-cultural applicability (i.e., culture fairness) of measures-which, however, is indispensable because the VIA framework conceives of character strengths as cultural universals (e.g., Church \& Lonner, 1998). Unlike the Big Five personality descriptors in the lexical-approach tradition, VIA is not based on the lexicon of the respective language. 
Given the quality of items, and particularly the small number of negatively keyed items in IPIPVIA, we were severely limited regarding the number of items available for creating balanced short scales. In selecting items from IPIP-VIA through expert ratings, we therefore pursued several interrelated goals in parallel: (1) Considerably shortening the scales to four items (i.e., 96 in total); (2) approximating unidimensionality; and optimizing (3) content validity and (4) the quality of item wording. We also paid heed to (5) cross-cultural applicability (or "fairness"). Finally, (6) the resulting item set should be balanced-keyed to control for acquiescence. In this way, we sought to address the previously discussed issues of IPIP-VIA items.

We used the expert ratings of all 252 IPIP-VIA items to select the best four items per strength. To become able to provide a sufficient number of content-valid-in particular reversed-items, our selection also considered IPIP items identified as potentially suitable for character strength assessment from other (non-VIA) IPIP sections. We computed indices of inter-rater agreement across all raters' judgments of item suitability. We then tested the unidimensionality of the selected item sets in four online samples (two from the U.K. and two from Germany) that were drawn specifically for this purpose. Because we had encountered some disagreement during the selection and because a few of the initial items and scales (subsequently termed Version A) were not fully satisfactory yet, we compiled Version B items and scales and collected similar evidence about unidimensionality (see Overview).

\section{Method}

Expert ratings and item selection criteria. To select items according to the five above-specified criteria, four professionals with expertise in personality/character assessment, scale development, and cross-cultural survey methodology screened the 252 IPIP-VIA items. They identified the bestsuited 96 IPIP items (i.e., four items for each of 24 character strengths) that would serve as the basis for translation and adaptation from English to German. The experts selected the items based foremost on how well the item content/wording matched the definition of the target strength (see Table 1). They were instructed to prefer concise items in simple language (clarity), and to avoid items whose content fit more than one character strengths (distinctness). Moreover, culture-specific 
connotations and idiomatic expressions were avoided to support cross-cultural equivalence. Each rater determined their preferred combination of the four most suitable items per scale (considered "essential"), with the option of marking one item per scale as "useful but not essential" (as a basis for establishing consensus). Ultimately balancing positive and negative items (acquiescence correction) required that discrepancies over which items to select had to be resolved by means of establishing post-negotiation consensus (see SOM_IPIP-VIA-R_Method_Item-Selection.pdf).

Based on the experts' inclusion/exclusion ratings, we computed the content validity index (CVI; Polit et al., 2007). For CVI, experts usually evaluate items according to four options, which are then collapsed into two categories: relevant or not relevant. In our case, the CVI at the item level (I-CVI) is directly computed as the proportion of "relevant"-judgments across raters; it reflects only the agreement about relevance (any agreement about non-relevance is not implied). Averaging I-CVI values of items selected into a scale yields CVI at the scale level (S-CVI). All CVI values range from 0 to 1 . Common rules of thumb suggest eliminating items with $\mathrm{I}-\mathrm{CVI}<.70$, revising items with $.70<\mathrm{I}-\mathrm{CVI}<.80$, and accepting items with I-CVI >.79. Others have suggested accepting items with $\mathrm{I}-\mathrm{CVI}>.70, .78$, or .80 (see Tilden, Nelson, \& May, 1990; Polit et al., 2007; and Davis, 1992, respectively). Yet others consider an average I-CVI as good (or excellent) content validity at the scale level if S-CVI > .80 (or .90; Polit et al., 2007). These rules of thumb are somewhat arbitrary and not universally applicable. ${ }^{6}$ Given our aim to select an a priori fixed number of four items considered to represent the best selection of items from an existing (i.e., fixed) item set with a set of four raters, we favored a cutoff of CVI $\geq .75$. To safeguard against unreliability of experts or chance agreement, we estimated inter-rater reliability (Hallgren, 2012).

\footnotetext{
${ }^{6}$ Despite (initially) independent experts, our procedure violates the assumption of independent judg-
} ments within scales, thus prevents the application of rigid rules and chance-corrections (e.g., Lawshe, 1975). 
Statistical analysis of closeness to unidimensionality. We then tested the unidimensionality assumption for each strength scale based on the initially selected items (IPIP-VIA-R, Variant A) in two samples (Sample 2 from the U.K. and Sample 3 from Germany). Moreover, we compared the initially selected items to a slightly modified item set (IPIP-VIA-R, Variant B) in two independent samples from the same countries (Samples 4 and 5). Item descriptives collected with Samples 2-5 can be found in SOM_IPIP-VIA-R_RQ2_Descriptives.pdf.

Using the FACTOR software (Ferrando \& Lorenzo-Seva, 2018), we ran an implementation of Horn's (1965) parallel analysis based on minimum rank factor analysis (MRFA; Timmermann \& Lorenzo-Seva, 2011), which exploits the (complete-case) polychoric correlation matrix for short scales based on a mere four (ordinal) items per strength. ${ }^{7}$ Again, 500 random correlation matrices, obtained through raw score permutation (Buja \& Eyuboglu, 1992), suggested the number of necessary dimensions. We ran MRFA under the constraint of extracting two dimensions at the most (which would reflect a potential method factor for acquiescence besides the substantive strength factor).

FACTOR also provided Explained Common Variance (ECV; Rodriguez al., 2016) as an index of unidimensionality. ECV et expresses the percentage of variance attributable to the (first) factor

${ }^{7}$ MRFA minimizes the amount of common variance left unexplained (Ten Berge, 1998; Ten Berge \& Kiers, 1991). MRFA is the only method that enables the percentage of ECV to be computed in the common factor model (Lorenzo-Seva, 2013). Other than principal axis factor analysis, which yields non-positive definite reduced matrices, MRFA returns strictly positive semi-definite reduced correlation matrices, so that all Eigenvalues are positive and derived coefficients computable (Lorenzo-Seva, 2013; Shapiro \& Ten Berge, 2002). Computing ECV from principal components is not appropriate with few variables and limited communality in short scales, as PCA confounds common with unique variance, so that loadings are inflated (Widaman, 1993). For the same reason, even with correctly specified categorical indicators and polychoric correlation matrices, running component-based MAP tests on short scales with few variables only shows a biasing tendency towards underfactoring (Garrido et al., 2011). 
common to all items (depending on the number of factors also termed the general factor). We inspected the so-called Mean of Item Residual Absolute Loadings (MIREAL) too, which expresses the average of the item loadings on a secondary factor (extracted with Promin rotation) and represents a general measure of departure from unidimensionality (Ferrando \& Lorenzo-Seva, 2018). ECV indicates the dominance of the first MRFA factor. MIREAL depicts a potential residual (second) MRFA factor. ECV should be high (> .60), and MIREAL should be low $(<.40)$, especially to prevent bias when using simple means or sum scores (see Table 5). The chosen cut-offs do reflect the small number of items, the non-redundant representations of strengths as well as the expected deviation from strict unidimensionality due to the balanced keying direction of items.

\section{Results}

Inter-rater reliability and content validity. An initial inspection showed $100 \%$ spontaneous agreement among experts about excluding 82 (33\%) of the 252 IPIP-VIA items. Another 53 (21\%) items were judged "essential" by merely one rater. By contrast, experts consensually judged 28 (11\%) items as suitable, and $43(17 \%)$ were preferred by a three-quarter majority among the raters. For the remaining $46(18 \%)$ items, there was a tie among the four raters. Never did we include an item into a strength scale if not at least half the experts considered it "essential" (or at least "useful" in one case-in the absence of a better reversed item for measuring Capacity for Love). With half the items unselected and only few reversed-keyed items in the pool, aiming at balanced four-item rather than six-item scales was the only realistic option with IPIP. Four items yield a reasonable compromise between reliability and efficiency for measuring strengths that reside at a similar level as personality facets, which the BFI-2 too assesses with four balanced items (Soto \& John, 2017a).

We used the intraclass correlation coefficient to quantify the agreement of the experts on their preferred selections (Hallgren, 2012; Koo \& Li, 2016). Using a two-way mixed effects model with raters as a fixed factor, the single-rater reliability was $I C C_{3,1}=.45$. The single-rater $I C C$ matches Fleiss's (1971) kappa $(\kappa=.45)$ and indicates moderate above-chance agreement between pairs of raters according to standards provided by Landis and Koch (1977). The reliability of the average judgment across all raters was $I C C_{3,4}=.76$. Given that raters could opt for different yet equally viable 
four-item sets, we consider this degree of reliability highly satisfactory. For illustrative purposes, after forming post-negotiation consensus and including non-VIA IPIP items to form balanced four-item scales (yielding Version A of IPIP-VIA-R), we coded the respective items as showing "perfect rater agreement", yielding agreement at $I C C_{3,1}=.54$ (single-rater), and $I C C_{3,4}=.83$ (average of raters). ${ }^{8}$

Thereafter, content validity ratings at the level of balanced scales (Variant A) amounted to an average S-CVI $=.84$ across 24 scales (range $=.63-1.00)$. For seven scales, S-CVI exceeded the set threshold of .75, and for another 13 scales S-CVI exceeded even all scales' average S-CVI ( $\geq$.85). Although four scales (Curiosity, Kindness, Originality, and Perspective) contained items that we consider suboptimal and that lowered S-CVI values below the set bar, we accepted these negotiated scales tentatively as the best possible set of items that can be obtained within the IPIP. Yet, out of 24 strength scales, 20 scales had good or very good content validity. Thus, Variant A represented substantially improved scales over the IPIP-VIA scales currently in use, whose items cannot all be recommended with good conscience. Still, over the course of the negotiation process, while considering alternative translations, and in light of the obtained statistical indices for unidimensionality (see next) and reliability estimates (see RQ3), we reasoned that five viable alternative items in Variant B scales existed (and an additional five substitute translations) that we compared in the next step.

Unidimensionality. To evaluate the dimensionality of all 24 character strengths, we focused on Samples 4 and 5, which allow for a direct within-sample comparison of Variants A and B. (For readers interested in the detailed first findings for Variant A scales based on our Samples 2 and 3, we provide the respective columns in Table 6, too.) Parallel analysis clearly indicated lower dimensionality for all 24 short scales than for the longer item sets (see RQ1). In the U.K. (Sample 4), parallel analysis suggested a single dimension to underlie eight Variant $A$ scales, and yet another three Variant B scales.

${ }^{8}$ The observed rater agreement for the preferred items prior to negotiation was $79 \%$ compared to $62 \%$ expected by chance, and it improved to $81 \%$ after negotiation (Freelon's, 2010, webservice ReCal3). 
For Citizenship/Teamwork and Kindness, two dimensions seemed possible in Sample 4 (but not so in Sample 2 before). In the remaining scales, not even a single factor could be reliably suggested after having opted for $95 \%$ statistical confidence in determining the likely number of factors with parallel analysis. In Germany (Sample 5), the majority of 19 Variant A and B scales supported the one-factor notion. None of the German scales appeared to be two-dimensional; yet for five Variant A scales (and three Variant B scales) there was a peculiar finding, namely that the number of suggested dimensions fell below one, while these values fluctuated across countries and samples.

Given the fluctuating number of suggested factors, we focused on ECV and MIREAL in Samples 4 and 5, whose values seemed rather comparable across countries and samples (see Table 6). ECV close to unity suggests a very strong common factor and supports an (at least essentially) unidimensional scale. Yet, with the built-in violation of unidimensionality (balanced item keying), an important question was if the secondary factor (method factor driven by acquiescence) would remain rather negligible. The common factor per scale explained reasonable percentages of variance, average $\mathrm{ECV}_{\mathrm{UK}}=.67$ and .68 , average $\mathrm{ECV}_{\mathrm{DE}}=.75$ and .76 , for scale Variants $\mathrm{A}$ and $\mathrm{B}$, respectively. The mean item-residual average loading values showed the presence of a constrained source of common variance in each strength scale, average $M_{I R E A L U K}=.39$ and .39 , average $M I R E A L_{D E}=.30$ and .29 , for scale Variants A and B, respectively. Often lower MIREAL values tended to be supported by higher ECV values, usually favoring Variant B over Variant A scales, as intended. Yet, direct strength scale comparisons were sometimes ambiguous, not always consistent across countries, and-especially when taking them in conjunction-did not clearly support the superiority of one variant over the other (e.g., Appreciation of Beauty and Excellence, Spirituality/Religiousness). Therefore, we deferred the ultimate choice between the Variants $A$ and $B$ until we had inspected the fit of proper measurement models, factor loadings, and scale reliability (RQ3-4).

\section{Discussion}

Based on expert ratings that achieved high inter-rater reliability, we were able to shorten the IPIP-VIA scales by about 50\% while concomitantly improving several measurement properties: unidimensionality, content validity, cross-cultural applicability, and balanced keying. Although measuring 
each character strength with four items was our aim from the outset (similar to the four items per facet in the BFI-2; Soto \& John, 2017a), the expert ratings also suggested that the IPIP hardly comprised more than four items suitable for achieving balanced character strength scales. Instead, experts agreed that most of IPIP-VIA items analyzed in RQ1 did not represent their respective strength adequately or exhibited other issues (e.g., lack of cross-cultural applicability). Selecting suitable, balanced item sets for each strength scale, however, yielded respectable content validity (S-CVI $\geq .75$ for 20 of 24 strengths).

Given the present scale lengths and sample sizes, MRFA-based parallel analysis was not trustworthy for determining the minimally required number of common factors (see Lim \& Jahng, 2019, for an explanation of underestimating the number of factors in short scales). Our reasoning was supported by the comparably consistent findings for ECV and MIREAL values and the changes therein when amending items to form Version B scales. Any scale achieved (at least essential) unidimensionality when judged by the reasonable percentages of common variance of underlying strength factors. Where the ECV-threshold of .60 was not passed by Variant A (Prudence and Appreciation of Beauty and Excellence), Variant B passed it. In general, MIREAL values showed that the scales guarded against severe violations of unidimensionality. Noteworthy, ECV values were higher and MIREAL values lower in the German compared to the U.K. samples-a finding that is, overall, in line with the pattern of parallel analysis. On average, the German item adaptations yielded closer approximation to unidimensional scales than the English source items. Therefore, we were satisfied with the obtained closeness to unidimensionality, especially since acquiescent responding as a secondary source of variance was not yet (statistically) controlled in these analyses (see RQ3).

Our finding that most existing VIA strength items fall short of accepted quality standards resembles the conclusions reached by Ng and colleagues (2017). These authors found that more than $50 \%$ of VIA-IS items had to be eliminated to achieve proper item loading patters for the 24 strengths. Their item selection was purely empirical and consisted in eliminating items with the poorest factor loadings iteratively. Our selection, by contrast, jointly optimized several substantive criteria. Together with further findings on the questionable representation of character strength cores by 
several items in extant VIA-IS scales (McGrath, 2014, 2019; McGrath \& Wallace, 2021), our results from RQ1 and the results on RQ2 caution against using unit-weighted scores to represent the VIA character strengths as measured with IPIP-VIA (and possibly with related measures of character strengths too). Unit-weighted scores derived from existing character strength measures are not unidimensional and contain several less-than-optimally worded items that threaten content validity and cross-cultural applicability. Given the lack of evidence for factorial validity (RQ1) and content validity (RQ2), our IPIP-VIA-based findings must have repercussions for the trustworthiness of previous analyses of associations among character strengths, identification of hierarchical structures, reliability estimates, construct validity, and predictive validity, let alone measurement equivalence across cultures.

\section{Research Question 3:}

\section{Do IPIP-VIA-R Measurement Models Achieve Good Fit?}

We sought to establish measurement models for IPIP-VIA-R that concomitantly would support choosing between the scale Variants A and B. Developing appropriate, character-strength specific measurement models is a hitherto neglected aspect in research on the VIA framework (but see Ng et al., 2017, and also Feraco et al., 2021, for increasing awareness of the problem). Selecting suitable IPIP-VIA items in such a way that all 24 short scales were balanced-keyed (i.e., two positively and two negatively worded items) allowed us to develop reflective measurement models for each character strength in which we modeled each character strength as a unidimensional latent variable and additionally controlled for respondents' acquiescent response style (henceforth ARS; Aichholzer, 2014; Billiet \& McClendon, 2000). As discussed earlier, ARS is a major source of bias in survey research, especially in research based on general population samples and in cross-cultural research (Lechner et al., 2019; Podsakoff et al., 2012; Rammstedt \& Farmer, 2013). Unless accounted for, individual differences in ARS can tarnish means and all covariance-based statistics of items and scales (McCrae et al., 2001). Consequently, failing to control for ARS also biases factor loadings, model fit statistics, and measurement invariance (e.g., De Beuckelaer et al., 2010; Schimmack et al., 2002). Controlling for 
ARS in our measurement model thus offered the chance to eliminate a likely source of (non-substantive) multidimensionality that is present in the character strengths scales (see RQ1-2).

One challenge in controlling for ARS is that unless the item set consists of perfectly balanced antonyms, an ARS factor may confound acquiescence with item-specific content. This is particularly true when only a few (e.g., 2 or 4) items are available from which to extract respondents' ARS. Fortunately, the validity of an ARS factor increases as the number of balanced and substantively heterogeneous items increases (De Beuckelaer et al., 2010; Aichholzer, 2014; Lechner et al., 2019). This is because ARS (i.e., agreement irrespective of item content and keying) is the only plausible source of indiscriminate agreement across a large and diverse set of items. We therefore capitalized on 96 balanced-keyed items in all IPIP-VIA-R scales to garner information about a respondent's ARS. Specifically, we computed a manifest ARS index as the ipsative (within-respondent) mean across all 96 balanced items. The ipsative mean scores across a large set of heterogeneous and balanced items reflects the systematic (dis-)agreement with items, irrespective of content. We then included this index as a control variable in the measurement model to remove ARS bias from the character strength indicators.

Figure 2 shows the tested CFA model for a given character strength. Four indicators reflected the latent character strength (identified with unit variance) with freely estimated factor loadings. Whereas strictly unidimensional models would deny the relevance of a method factor for balanced item sets, we controlled for acquiescence by regressing each item on the ARS index. Manifest ARS indices typically correlate very highly with latent ARS variables (Billiet \& McClendon, 2000), yet specifying a secondary latent ARS variable for each strength would require longer strength scales. Because the variance of the ARS index is given and fixed, we estimated the regression weights freely while constraining them to equality across all four items of each scale. Hence, the impact of ARS can differ per strength scale ("scale-specific acquiescent responding"; Greenleaf, 1992; Schimmack et al., 2002; Weijters et al., 2010).

We used these novel measurement models to establish whether each of the 24 character strength short scales can be modeled as a unidimensional latent variable (after controlling for ARS) 
and to compare the scale Variants $A$ and $B$ in order to decide on the final versions of the IPIP-VIA-R strength scales. Moreover, the models provide the foundation for our subsequent analyses of the scales' reliability, validity, and measurement invariance (RQ4-7).

\section{Method}

We estimated CFA model parameters for Samples 2-5 with robust maximum likelihood estimation (MLR; Rhemtulla et al., 2012). We handled the few missing item responses with Full Information Maximum Likelihood (FIML) under the assumption of missingness at random (MAR). We judged the fit of the measurement models by the $\chi^{2}$-test with $d f$ model degrees of freedom and the resulting goodness-of-fit indices. At large sample sizes, $\chi^{2}$ will indicate statistically significant deviations of the model-implied from the empirical variance-covariance matrix even if misfit is trivial. In line with current guidelines (e.g., Kline, 2011), we therefore considered the largely sample-size independent Comparative Fit Index $(\mathrm{CFI})$, the parsimony-rewarding Root Mean Square Error of Approximation (RMSEA), and the Standardized Root Mean Square Residual (SRMR). Previously suggested cutoff heuristics cannot be applied to very short scales and complex models (Marsh et al., 2004), especially with few $d f$ remaining and with low factor loadings (e.g., due to rather heterogeneous item content; Heene et al., 2011), as is partly true for the current strength short scales. Therefore, we considered models as tenable when $\mathrm{CFI} \geq .90, \mathrm{RMSEA} \leq .10$, and $\mathrm{SRMR} \leq .08$ (see Table 5$).{ }^{9}$

${ }^{9}$ Using stringent cutoffs rigidly is disavowed because models may get rejected that are, in fact, appropriate (Marsh et al., 2004). Fit heuristics have their merit when tested models reflect the simulation conditions from which cutoffs were derived. However, RMSEA is inflated with a low number of items (e.g., $k=4$ ), even when a model is correctly specified (Kenny et al., 2015; Kenny \& McCoach, 2003; Shi et al., 2019), and CFI is too low if the null model has some plausibility, that is, when conceptual breadth and item heterogeneity restricts inter-item correlations to some extent (Kenny et al., 2015). 


\section{Results}

Model Fit and Factor Loadings of IPIP-VIA-R Strength Scales: Variants A and B. We first present the average fit indices for Version A scales as obtained with Samples 2 and 3, because these models, their respective fit indices, and their respective factor loadings constituted the statistical starting point for engaging with Version B scales. (We provide details about measurement models, goodness-of-fit indices, and standardized loadings for all samples, scales and their variants in SOM_IPIP-VIA-R_RQ3_Measurement-Model-Fit-\&-Loadings.pdf.) Across all 24 strengths, we obtained very good average values of $\mathrm{CFI}=.970$ and $.949, \mathrm{RMSEA}=.056$ and .072 , and SRMR $=.044$ and .050 for the U.K. and Germany, respectively. According to the conservative (but non-significant) $\chi^{2}$-test, eight scales even fit perfectly in both countries, and another five scales in the U.K. Considering fit heuristics, all scales passed the SRMR cutoff, and nearly all scales passed the CFI threshold except for EQU, LOV, and SPI. According to RMSEA, which tends to be biased in short scales, 14 scales were suitable in both countries, yet eight scales flagged in at least one country.

All factor loadings were in the expected direction and standardized loadings were, on average, appropriate for short scales covering character strengths in breadth. Absolute loadings across all 24 scales averaged to the following means of $\lambda(1-4)=.51-.60$ in the U.K. and .54-.61 in Germany. The minimum and maximum $\lambda$ s encountered for any specific items were .10 (APP \#4 in DE) and .83 (SPI \#4 in DE). Eight scales provided at least one item with a low loading $(\lambda<.40)$, which prompted us to consider alternative items (or translations, when only German loadings were conspicuous). ${ }^{10}$ Love of Learning seemed problematic, too, because we obtained relatively poor RMSEA and CFI values across both countries. We sought to better translate the item FOR \#1 ( $\left.\lambda_{D E}=.37\right)$. We did not replace the

${ }^{10}$ Flagged items were APP \#4 (UK \& DE), CIT \#2 and \# 4 (DE), EQU \#4 (DE), GRA \#4 (UK), PRU \#2 and \#4 (UK), SEL \#1 and \#2 (UK) and \#4 (DE), SPI \#2 (UK and DE), and ZES \#2 (UK). 
item GRA \#4 $\left(\lambda_{U K}=.34\right)$, as its translation worked flawlessly $\left(\lambda_{D E}=.69\right)$ and $\chi^{2} u k$ was far from being significant.

When comparing the model fit of Variant A scales in Samples 2 and 3 against those in Samples 4 and 5 , the scale quality was largely similar. For instance, two Version A scales we had flagged (Love of Learning and Spirituality/Religiousness) had problematic model fit in all samples. Given the highly comparable findings, we deemed contrasting and assessing scale Variants A and B within Samples 4 and 5 useful (and potentially better than comparing Variant A from Samples 2 and 3 with Variant B from Samples 4 and 5). Regarding this within-sample comparison, the average fit across all Version $A$ scales was good or very good, $\mathrm{CFI}=.980$ and $.966, \mathrm{RMSEA}=.055$ and .069 , and SRMR $=.041$ and .058, for the U.K. and Germany, respectively. Similar good fit resulted, on average, when analyzing Version B scales, $\mathrm{CFI}=.975$ and $.964, \mathrm{RMSEA}=.060$ and .068 , and SRMR $=.042$ and .055 , for the U.K. and Germany, respectively (see Tables B2 and B3 in SOM_IPIP-VIA-R_RQ3_Measurement-Model-Fit\&-Loadings.pdf). With non-significant $\chi^{2}$-tests, 12 and 9 Version A scales as well as 13 and 8 Version B scales obtained perfect fit in the U.K. and Germany, respectively. The remaining models had a $\chi^{2}-$ value conventionally labelled as statistically significant, but the model fit indices fell in a range conventionally labelled as sufficient to very good.

Considering jointly the resulting model fit and factor loadings, some Version B scales (e.g., Appreciation of Beauty and Excellence with nonsignificant $\chi^{2} u k$ ), but not all Version B scales (e.g., Forgiveness: nonsignificant $\chi^{2}{ }_{D E}$ in Version $A$, but significant in Version B), worked better. Despite overall good model fit, the values did not consistently favor all Version B scales. Given the equivocal results regarding these scale variants, we based our decision about which variant to retain in the final version on both linguistic considerations and all empirical evidence on dimensionality (ECV, MIREAL) from RQ2 as well as the CFA model fit and factor loadings. We additionally considered scale reliability (presented next in RQ4). On this basis, we preferred three Variant B scales (APP, LOV, ZES) yet retained two Variant A scales (PRU, SPI) because the corresponding Variant B scales had several unacceptably low factor loadings in Germany. Of the four scales with either one (EQU, FOR, SEL) or two (CIT) alternative item translations, we always preferred Variant B. For details, see SOM_IPIP-VIA- 
R_RQ3_Item-scale-variant_Selection-Criteria.xlsx (and the accompanying documentation SOM_IPIPVIA-R_RQ3_Item-scale-variant_Selection-Criteria.pdf). Table 8 presents the resulting final 96 items of the refined IPIP-VIA-R short scales.

Model Fit of Final IPIP-VIA-R Strength Scales. We provide a summary of the findings for the final IPIP-VIA-R scales (see Table 7). For Germany, eight scales showed excellent CFA model fit without a statistically significant $\chi^{2}$-deviation (all $\chi^{2} \leq 8.69$ and $p>.12$ ). Likewise, the same eight scales (plus another five scales) in the U.K. similarly showed near-perfect fit $\left(\chi^{2} \leq 9.82, p>.08\right)$. Model fit fell in a range that would be conventionally labeled as sufficient to very good: Across all 24 strengths, we found average values of CFI $=.980$ and .965, RMSEA $=.055$ and .070 , and SRMR $=.041$ and .059 , for the U.K. and Germany, respectively. For all models with significant $\chi^{2}$-test, except for Spirituality/Religiousness, the majority of the three fit indices fell within acceptable limits. With the caveat that it might be inflated for short scales, RMSEA flagged the fit of the model for Hope as potentially problematic consistently in both countries. Similarly, RMSEA consistently leaned towards the upper boundary for the Leadership scale.

\section{Discussion}

These findings demonstrate that unidimensional measurement models with ARS control showed good fit for most of the 24 character strengths in four samples representing two countries and languages. This suggests that our attempts to obtain unidimensional measures of the character strengths by discarding items that fell short of conventional standards in terms of linguistic quality and content validity were successful. In line with the findings from RQ2, our results also attest to the 
importance of modeling ARS in order to obtain well-fitting models that provide a solid foundation for testing reliability, measurement equivalence, and construct validity. ${ }^{11}$

Only three of the measurement models (for SPI, HOP, and LEA) did not achieve sufficiently good fit across all the fit indices and/or had at least one item with a lower factor loading. One might speculate that the limits of better measurement within the IPIP-VIA framework become tangible here. However, the models for these three strengths might still be improved by permitting correlated errors that may be expected when psychological tests are shortened, because with small numbers of items the error score estimates are based on increasingly insufficient true score estimates (Beauducel \& Leue, 2014). We will explore this possibility before testing cross-cultural equivalence (RQ5) because, rather than simply optimizing fit within countries, any model adjustment should be applicable across countries.

\section{Research Question 4:}

\section{How Reliable are the Final IPIP-VIA-R Strength Scales?}

Although the latent measurement models established in RQ3 showed adequate fit and constitute an optimal way of modeling character strengths as part of structural equation models (SEM), in practice many researchers will use manifest scale scores for character strengths. When manifest scale scores (as opposed to latent-variable modeling or plausible values) are used, measurement precision is an essential consideration. Next, we therefore shed light on the reliability of the 24 final IPIPVIA-R short scales. Because "the choice of a single statistic to summarize the accuracy of an

${ }^{11}$ While specifics are beyond the present scope, a cursory inspection of unidimensional models that omitted the ARS index resulted in significant $\chi^{2}$-tests for all Variant A and B scales in the U.K (Sample 4) and poor fit, mean $\mathrm{CFI}=.63, \mathrm{RMSEA}=.26, \mathrm{SRMR}=.08$; similarly, in Germany (Sample 5), mean CFI $=.85, \mathrm{RMSEA}=$ $.14, \mathrm{SRMR}=.04$. Dropping ARS for a few scales where model fit might suffice on the surface yields inconsistent measurement models across countries and IPIP-VIA-R scales. 
instrument is not the best report that can be made" (Cronbach \& Shavelson, 2004, p. 414), we computed several different reliability indices (for cutoff heuristics, see Table 5). Our focus was on internal reliability indices that are based on the appropriate measurement models specified in RQ3 (i.e., omega) and on test-retest reliability. For comparability with prior research, we also report more traditional reliability estimates (e.g., Cronbach's alpha), even though we note that some of these reliability estimates do not do justice to the measurement models for the character strengths. Finally, we also computed test-retest reliability. We computed each reliability estimate separately for each country. We present a summary evaluation of the final IPIP-VIA-R item sets (Samples 4 and 5; for scale-specific details, see Table 9; for statistical details and reliability outcomes in Samples 2 and 3, see SOM_IPIP-VIA-R_RQ4_Reliability.pdf).

What is considered "acceptable" or "sufficient" reliability depends on the research purpose. Population surveys often require cost-effective short scales, resulting in a trade-off between saving resources and aiming for high psychometric quality (e.g., Kemper et al., 2019). Whereas longer, more reliable scales are required for precise individual diagnostics, short surveys often compensate lower reliability estimates by means of larger sample sizes (Rammstedt et al., 2020). Also note that reliability is study-specific, as it depicts how reliable the individual differences are in a given sample of participants. Cutoff criteria for evaluating reliability need to reflect the different purposes of measurement (Nunnally \& Bernstein, 1994). Short scales, especially designed to preserve the substantive breadth of the target constructs, often have reliabilities that fall below conventional thresholds; despite their lower reliability, they often predict life outcomes almost as well as longer scales (e.g., Rammstedt et al., 2020; Soto \& John, 2019; Thalmayer et al., 2011). Considering the brevity of the IPIP-VIA-R scales, we therefore accepted reliabilities of $.60<\rho<.70$ as sufficient for most research purposes, $.70<\rho<.80$ as good, and values $\rho \geq .80$ as excellent.

\section{Method}

We computed the following reliability indices. Irrespective of the specific estimate, reliability ( $\rho)$ according to classical test theory reflects the proportion of true score variance in the observed score variance: 


$$
\rho=\frac{\sigma_{\tau}^{2}}{\sigma_{\tau}^{2}+\sigma_{\varepsilon}^{2}}
$$

Cronbach's Alpha ( $\alpha$ ). Alpha is the most widely used measure of internal consistency. For this reason, we report it here. However, coefficient alpha provides merely a lower-bound estimate for the reliability of the unit-weighted scale score. Furthermore, it is based on assumptions that are regularly violated in personality assessment, namely, strict unidimensionality, absence of residual correlations, and an essentially tau-equivalent model (i.e., identical factor loadings for all items; Cho \& Kim, 2015; Graham, 2006). When these assumptions are violated, $\alpha$ potentially underestimates or, more rarely, overestimates scale reliability (Raykov, 1997, 1998, 2001; Sijtsma, 2009). We computed $\alpha$ for each scale after recoding the reversed-keyed items.

McDonald's Omega ( $\omega)$. A reliability estimate that is more appropriate for tau-congeneric measures-and hence for most personality measures-is McDonald's Omega (McDonald, 1999). Different from $\alpha, \omega$ does not assume tau-equivalence and can be generalized to handle violations of unidimensionality, providing a more realistic reliability estimate (e.g., Dunn et al., 2014). We estimated $\omega$ based on the accepted measurement models developed in RQ3 via Raykov's (1997) phantom-factor method, while accommodating the categorical nature of the ordinal indicators by a robust WLS estimator (WLSMV; B. Muthén et al., 1997; for details, see SOM_IPIP-VIA-R_RQ4_Reliability.pdf). This provides more realistic and trustworthy reliability estimates than coefficient alpha.

Test-retest reliability $\left(\boldsymbol{r}_{\mathrm{tt}}\right)$. Test-retest reliability provides an estimate of the replicability of test scores when a test is administered twice. We estimated $r_{\mathrm{tt}}$ as the linear correlation between each strength, measured at two time points (Guttman, 1945). Different from $\alpha$ and $\omega, r_{\mathrm{tt}}$ reflects the temporal consistency, that is, the reliability of all temporally stable sources of variance in the scale score (i.e., true score variance but also other stable influences such as acquiescent response style). For estimating the test-retest-reliability $r_{\mathrm{tt}}$ of the 24 character strength scales, we re-invited participants from samples 4 and 5 . The retest surveys consisted exclusively of the strength scales. The median time-lag amounted to 20 days $\left(M_{\mathrm{DE}}=19.76, S D=1.30 ; M_{\mathrm{UK}}=20.50, S D=1.13\right)$. After quality filtering (and listwise deletion), the analytical retest sample rested on $n=224\left(n_{\mathrm{DE}}=117, n_{\mathrm{UK}}=107\right.$; originally 
244 participants with $N_{\mathrm{DE}}=124$ and $N_{\mathrm{UK}}=120$ ). The time-lag of three weeks represents a good tradeoff between incurring inflated reliability due to memory effects and underestimation due to trait volatility.

We also computed the correlation between the latent strength variables across the two measurement occasions to provide a glimpse at the stability of reliable true score variance (which includes trait variance, but also stable state variance across three weeks). With its help it is easier to interpret the test-retest reliability coefficients, which entail true trait changes and state fluctuations across time, and which are also attenuated by measurement error. The retest correlation between the latent strength variables represents a natural boundary for the retest reliability of manifest scale scores.

Additional reliability coefficients. In addition to our three main reliability coefficients, we computed four additional coefficients: Average Inter-Item Correlation (AIC), Average Variance Extracted (AVE), Factor Determinacy Index (FDI), and construct replicability or generalized H-Index (gHI). These indices provide complementary information and may be of interest to some researchers and in specific research scenarios. Descriptions and results for these additional indices are also available in SOM_IPIP-VIA-R_RQ4_Reliability.pdf.

\section{Results}

Cronbach's Alpha $(\alpha)$. Average $\alpha$-values were $M_{\mathrm{UK}}=.58(0.056)$ and $M_{\mathrm{DE}}=.63(0.075)$. Though some scales fared better than average (e.g., Humor, Industry/Perseverance), these values include "disappointing" coefficients even from the perspective of social surveys. However, apart from looking at short scales, $\alpha$ is likely to underestimate the reliability of IPIP-VIA-R because these scales do not meet $\alpha$ 's restrictive assumptions as noted earlier.

McDonald's Omega ( $\omega)$. As expected, $\omega$ was consistently higher than $\alpha$ (except for the SelfRegulation scale in Germany) and reached adequate levels, $M_{\mathrm{UK}}=.76(0.047)$ and $M_{\mathrm{DE}}=.75(0.066)$. Almost one third of the strength scales had even highly satisfactory values $(\omega>.80)$ in both countries, 
driving about three quarters of the variance in the unit-weighted composite score. ${ }^{12}$ These findings suggest that the primary factor in each IPIP-VIA-R scale is substantive, but this fact is concealed by $\alpha$.

Test-retest reliability $\left(r_{\mathrm{tt}}\right)$. The average $r_{\mathrm{tt}}$ for manifest scale scores was $M_{\mathrm{UK}}=.66(0.076)$ and $M_{D E}=.74(0.066)$. This is a realistic picture about what reliability to expect from manifest IPIP-VIA-R short scales (see McCrae et al., 2011). The German values are in line with the $\omega$ estimates, whereas $r_{\mathrm{tt}}$ in the U.K. was often lower than corresponding $\omega$ values. Note that even after accounting for measurement error, the correlations for the latent variables fell clearly below 1.00 (in nearly all cases), with $M_{\mathrm{UK}}=.83$ (0.106) remaining lower than $M_{\mathrm{DE}}=.90$ (0.059). The discrepant U.K. findings might be attributed to worse item wordings than we achieved for the German adaptations, alternatively to lower sample quality or other reasons responsible for higher state fluctuations then in Germany, unless one is willing to speculate on a theoretical level about less stable character strengths in the U.K. (i.e., culture-specific reasons) or unless short-term historical effects affect one country but not the other (i.e., political ruptures).

\section{Discussion}

Overall, these results suggest that the IPIP-VIA-R short scales achieved sufficient levels of reliability in the present samples. Especially in view of the brevity of the scales and the fact that we selected content-valid IPIP items while preserving the substantive breadth of each character strength, many reliabilities were highly satisfactory. This applied to omega (the most appropriate reliability coefficient for these scales) and test-retest reliabilities.

${ }^{12}$ Comparing $\omega$ to gHI values (see SOM_IPIP-VIA-R_RQ4_Reliability.pdf) shows that using unit-weighted $(\omega)$ instead of ideally weighted composites $(\mathrm{gHI})$ is usually not detrimental to the reliability of IPIP-VIA-R short scales. We observed little differences. The maximum discrepancies were .15 for Social Intelligence in Germany, and .21 for Self-Regulation in UK. 
Regarding the (less suitable) alpha reliabilities, let us compare IPIP-VIA-R scales to BFI-2 facet scales (Soto \& John, 2017a, 2017b), which comprise four items with balanced keying for each of three domain-specific Big Five facets (see RQ6). In both countries, we observed somewhat lower values than reported for the BFI-2 facet scales of a U.S.-dominated Internet validation sample, range = $.59-.83(M=.74 ;$ Soto \& John, 2017a). Comparable $\alpha$ values resulted for the BFI-2 facet scales in a German Internet sample, range $=.56-.84(M=.73$; Danner et al., 2019). Evidently, compared to IPIPVIA-R, the BFI-2 scales meet alpha's assumptions better, because the items have rather narrow wordings and constitute rather homogeneous or factor-pure scales. By contrast, when looking at $\omega$, the IPIP-VIA-R scales played in the same league as the German BFI-2 facet scales, range $=.66-.87(M=$ .78; Danner et al., 2019). Again, when looking at the retest-reliability coefficients, which do not assume homogeneous items in the scales, we obtained values (predominantly in Germany) that are comparable to those reported for the German BFI-2 facets, range $=.58-.85$ ( $M=.78$; Danner et al., 2019).

Whereas past reliability estimates for character strength scales cannot be trusted because their assumptions have not been tested, the internal consistency $(\omega)$ of IPIP-VIA-R short scales was satisfactory in the U.K. and in Germany, and retest reliabilities of scale scores $\left(r_{\mathrm{tt}}\right)$ confirmed this optimistic picture for Germany, while U.K. participants responded less temporally consistent. Not all scales performed equally well on all reliability estimates, though, and a few scales clearly had lower internal consistency (or retest reliability) than others. Especially for research in which the character strengths are used as predictors of life outcomes, the unreliability of scale scores can lead to attenuation bias, which requires statistical disattenuation. For such scenarios, we also recommend latentvariable models that control for method and error variance (see Lechner et al., 2021, for an overview).

\section{Research Question 5:}

\section{Do IPIP-VIA-R Scales Function Equivalently Across Cultures?}

Measurement invariance is a key prerequisite for research studying a construct of interest in different groups such as cultures-one simply cannot compare chopsticks with forks (Chen, 2008). 
Measurement invariance ensures that respondents with the same true standing on an underlying latent dimension have the same expected score on observed variables (Meredith, 1993). Measurement non-invariance introduces bias into cross-cultural comparisons of means, variances, or relations with predictors and outcomes.

Even though Peterson and Seligman (2004) selected character strengths for their VIA framework based on whether these strengths were universally acknowledged across different cultures, few studies have tested the measurement invariance of character strength scales across different cultures and languages. Some studies investigated the higher-order structure of character strengths in different cultures (e.g., McGrath, 2014, 2015, 2016; Ng et al., 2017) or structural relations among different strength scales (McGrath, 2016), yet these studies did not examine the cross-cultural invariance of each strength scale.

Personality and value inventories often show limited cross-cultural generalizability (e.g., expressions of spirituality; see MacDonald et al. 2015; Piedmont, 2007). Especially the comparability of latent means is often hard to achieve across cultures. Thus, it is important to establish whether our newly developed IPIP-VIA-R scales achieve levels of invariance that allow for meaningful comparisons across countries. Based on our measurement models developed in RQ3, we tested the configural, metric, and scalar measurement invariance of the 24 character strength scales across the U.K. and Germany (for details, see method). For well-constructed instruments, configural and metric invariance levels usually do not pose large challenges. The real challenge is scalar invariance. With some item adaptations affecting item extremity (or vague quantifiers) to improve measurement quality in German items, we did not expect to find scalar invariance for all the scales.

\section{Method}

We tested measurement invariance by fitting 24 multi-group confirmatory factor analysis (MGCFA) models with robust maximum likelihood estimation (MLR). Establishing measurement invariance involves testing the fit of hierarchically nested, increasingly restricted measurement models across groups (Meredith, 1993; Vandenberg, \& Lance, 2000). If a specific level of measurement invariance holds, the next (higher) level can be inspected, until invariance testing of structural parameters 
becomes possible (Meredith, 1993). All aspects of the factor-analytic measurement model can be tested by introducing more and more equality constraints in MGCFA: Following Putnick and Bornstein (2016), the four measurement invariance steps that can be considered are: (1) configural, equivalence of model form (same item-factor configuration); (2) metric (weak factorial), equivalence of factor loadings (same units of measurement in scaling the construct); (3) scalar (strong factorial), equivalence of item intercepts or thresholds (absence of item difficulty bias); and (4) residual (strict or invariant uniqueness), equivalence of items' unique variances (amount of unsystematic error). When unfair measurement can be ruled out, one might proceed to testing structural invariance (factor means, variances, and covariances), if these are substantive questions of interest.

We limited our present analyses to the first three of these steps (configural, metric, and scalar) because scalar invariance is all that applied researchers typically seek. Scalar invariance ensures that constructs have the same meaning across cultures and allows for comparisons of latent variances, covariances (correlations), and means and across cultures. If a specific level of measurement equivalence (e.g., scalar) does not hold, certain comparisons (e.g., of latent means) will not be permissible or at least be biased (Brown, 2015; Chen, 2008; Meredith, 1993; Steinmetz, 2013; Wu et al., 2007). In such cases, fitting a partial metric or scalar invariance model may still be possible: Even if some loadings and/or intercepts are unequal across groups, others may be invariant and one may achieve good model fit by lifting the equality constraints across groups of a few "problematic" (i.e., non-invariant) items (Byrne et al., 1989; Vandenberg \& Lance, 2000).

To establish what level of invariance each scale could attain, we inspected 1 ) robust $\chi^{2}$ statistics, scaled for deviation from normality (Satorra \& Bentler, 2010); 2) fit-heuristics (CFI, RMSEA, SRMR; Bentler, 2007) considered less susceptible to sample size than $\chi^{2}$ and $\Delta \chi^{2}$-tests, and 3) information criteria that favor models with a better accuracy-parsimony tradeoff (BIC; Schwarz, 1978). Instead of relying on a single criterion, we considered the whole profile of indices. We observed usual guidelines for model fit, which equal the cutoffs suggested for configural invariance (Hu \& Bentler, 1999; Rutkowski \& Svetina, 2014). We followed Chen's (2007) criteria and accepted the metric level if the decrease in model fit in $\triangle$ CFI was not larger than .010 in combination with $\triangle \mathrm{RMSEA}<.015$ (or 
$\Delta$ SRMR < .030; cf. Cheung \& Rensvold, 2002). Again, following Chen (2007), we accepted scalar equivalence if $\Delta$ CFI differences $<.010$ combined with $\triangle R M S E A<.015$ (or $\triangle S R M R<.010$ ). For the ultimate decision about accepting or rejecting an invariance level in ambiguous cases, we preferred information criteria over cutoff heuristics (see also Fan \& Sivo, 2009; Marsh et al., 2004). When comparing invariance level, lower BIC values indicate the better model when considering accuracy and parsimony jointly. Yet, $\triangle \mathrm{BIC} \leq 2$ is hardly worth mentioning, $\triangle \mathrm{BIC}$ between 2 and 5 is first evidence for one model being superior, but only a difference larger than 5 (or 10) is strong (or very strong) evidence that suggests discarding one of the models, that is, either the stricter or the more lenient invariance level (Raftery, 1995). For details on the approach, see SOM_IPIP-VIA-R_RQ5_Measurement-Invariance.pdf.

\section{Results}

Table 10 shows the outcomes of the testing procedure. BIC and fit heuristics mostly agreed. If not, then indices exceeded Chen's (2007) thresholds only marginally. If there was disagreement between $\mathrm{BIC}$ and fit heuristics, we accepted the higher invariance level if lower BIC supported the more parsimonious model (which we indicate by presenting the accepted model in parentheses). However, we used double parentheses for accepted invariance levels, if BIC increased marginally, that is, when BIC provided some (but not yet compelling) evidence against the more parsimonious (higher invariance) model.

Configural invariance. MGCFA models with the same configuration for all 24 scales (as established in RQ3) across countries fit well, confirming configural invariance. Yet, as already announced in RQ3, we first had to introduce one pair of correlated item residuals for three scales: Hope*, Leadership*, and Spirituality/Religiousness* (denoted by an asterisk in Table 10). Morizot et al. (2007) advised that residual correlations above $|r|>.20$ be flagged as evidence of a factor solution not adequately accounting for the data structure, thus signaling deviation from unidimensionality. The newly introduced residual correlations were meaningful and conveyed item overlap (in content or semantics; see Table 8): Hope items \#1 and \#4 reflect pessimism. Leadership items \#1 and \#2 reflect overlap 
regarding teamwork. Spirituality items \#2 and \#3 reflect meaning in life. After establishing fitting configural multigroup models, we proceeded to testing loading invariance.

Metric invariance. Loadings were invariant for 23 out of 24 scales. The sole exception was Gratitude, which required one free loading to achieve partial metric invariance. Mplus modification indices (ModInd > 10) suggested relaxing the equality constraint on the first Gratitude item ("expressing thanks"). One can speculate here about cultural display rules that might govern "expressing thanks". After relaxing the equality constraint on the item, the (unstandardized) loading was 0.70 in the U.K. and 0.36 in Germany. Given that only one of the 96 items showed non-invariance, we judged metric invariance to be a given.

Scalar invariance. Encouragingly, scalar invariance held for 11 out of 24 scales (see Table 10), despite minor BIC increases for two of these 11 scales. For the remaining 13 scales that did not attain full scalar invariance, we tested whether partial scalar invariance was attainable. With at least two invariant item intercepts, it is still possible to compare means of latent variables. We did this by freeing the equality constraint on the intercept with the highest modification index (usually when ModInd >10); if this did not yield an acceptable model, we freed a second item intercept. For seven out of 13 scales, partial scalar invariance resulted after freeing one intercept. The remaining six scales profited from a second free intercept, though for two scales-Self-Regulation and Citizenship/Teamwork - the decision to release a second parameter might be considered subjective: BIC improved (by $-2)$ in the presence of a moderate CFI increase $(\leq+.016)$, while Modlnd did not stick out $(<10)$.

\section{Discussion}

All 24 IPIP-VIA-R scales achieved at least partial invariance between Germany and the U.K. This high degree of comparability across the two countries and languages is remarkable and shows that the efforts to ensure comparability during the translation and adaptation process were successful. Partial scalar implies that the meaning of the 24 character strengths is identical in both countries. Moreover, it implies that both covariance-based statistics (e.g., factor variances and correlations; see RQ6) and latent means can be validly compared across countries. For the 11 scales that achieved full scalar invariance, cross-country comparisons are permissible even with the manifest scale scores. For 
the 13 scales that achieved partial scalar invariance (six scales with one noninvariant intercept; seven scales with two noninvariant intercepts), mean-level comparisons should only be done in latent-variable framework, although the resulting bias when using the scale scores is likely to be small.

Overall, then, findings from measurement invariance testing are encouraging. They support the suitability of the IPIP-VIA-R scales for cross-national research that, perhaps, generalizes to other English- and German-speaking countries and beyond. Before it can be claimed that the 24 character strengths represent cultural universals, research should test measurement invariance across a larger and more diverse set of cultures and languages.

\section{Research Question 6:}

\section{Are IPIP-VIA-R Scales Construct-Valid? A Nomological Network Perspective}

Having closely defined the empirical framework for how to measure the strengths, we evaluated the construct validity of IPIP-VIA-R character strengths by locating them in a comprehensive nomological network (Cronbach \& Meehl, 1955) with Big Five personality traits and basic human values. Whereas the relevance of Big Five is undisputed, the fundamental value circle proposed by Schwartz $(1992,1994)$ is equally relevant for value-laden character strengths, though research on associations with VIA strengths is even scarcer. For our comprehensive approach, we first introduce our hypotheses about VIA strength associations with fundamental personality domains before we elaborate on the structure of value orientations and their relevance for VIA strengths.

Relations between Character Strengths and Personality Traits. The five-factor model of personality is one fundamental model of basic traits: Extraversion, Agreeableness, Conscientiousness, Neuroticism/Negative Emotionality (or reversely: Emotional Stability), and Openness/Open-Mindedness (Goldberg, 1990; John et al., 1988). There is ample evidence that the Big Five reflect enduring dispositions that have a biological basis and manifest themselves in behavioral patterns. Though distinct trait expressions may be observed across cultures, researchers established five factors that typically underlie personality descriptors in numerous languages. Some researchers prefer including a sixth factor, whose label may depend on the criteria for lexical studies (e.g., Ashton \& Lee, 2020; Ashton et al., 2004; Saucier, 2009; for a more nuanced (and hierarchical) view of personality traits, see 
Saucier \& lurino, 2020). We hypothesized for each of the 24 strengths about their most relevant Big Five domain.

The Big Five domains allow a reliable orientation within the dimensional sphere of personality (at least in Western samples). Several facets underlie each factor (Costa \& McCrae, 1992, 2008), and in the case of the cross-culturally validated BFI-2 inventory (see Methods), three facets represent each Big Five domain (Soto \& John, 2017a). The facet level provides highly specific traits (see Table 2a). Measured with fewer items than the domains, they are typically less reliable. Yet, combining multiple facets in multivariate regression, they are a powerful tool for increasing the predictive accuracy beyond that gained from using general domain scores (see also Mõttus et al., 2020). We therefore attend to predictive models that allow a comparison of associations of strengths with the Big Five framework both at the domain and at the facet level.

As demonstrated by earlier work (McGrath, 2014; Noftle et al., 2011), strengths are related to, but not identical with, the Big Five domains. As we outlined earlier, VIA character strengths reside at a lower (i.e., more fine-grained) level of abstraction than global Big Five personality traits, and also at a lower level than the four higher-order value clusters in Schwartz's (1992) hierarchical model of basic human values. Instead, character strengths reside on a similar level of abstraction as personality facets (though character strengths appear to be slightly broader than personality facets; cf. internal consistency in RQ4), and some individual strengths may partly measure the same traits as some personality facets (McGrath et al., 2020). Consistent with a (non-cognitive) skill view, character strengths are amenable to change, much like skills develop over the lifespan (Denissen et al., 2019; Specht et al., 2011).

We hypothesized a priori about the strongest relationships to any of the Big Five domains for each character strength, thereby providing a confirmatory test of this part of the nomological network. Given the longstanding dearth of research on relationships between strengths and specific personality facets at the time of our study (but see the recent paper by McGrath et al., 2020, and the preprint by Vylobkova et al., 2021) and because it exceeds the scope of this study, we did not make any predictions for facets, rendering this part of the analysis exploratory in nature. Note that 
Peterson and Seligman's (2004) Tables 3.7 and 3.10 on the correspondence between character strengths and the Big Five framework and the Schwartz values remained incomplete, as they did not suggest for every character strength its most likely associate. Therefore, two raters in our research team hypothesized for each character strength its strongest positive or negative association with one the Big Five domains (and with the Schwartz values, as discussed below). Raters agreed for 19 out of 24 strengths; disagreement was resolved through discussion. The one exception was Spirituality/Religiousness, because of its blend that might compound "subjective spirituality" and "orthodox religiosity": Spirituality tends to be associated with high Openness, and Religiousness with low Openness (see Piedmont, 2007). Hence, raters disagreed on a dominant Big Five associate, but accepted two domains as validity evidence (see SOM_IPIP-VIA-R_RQ6_Nomological-Net-Hypotheses.xIxs).

Character strengths and basic human values. According to the theory of basic human values, ten motivationally distinct basic values represent core values that are culturally "shared conceptions of what is good and desirable in the culture" (Schwartz, 2006, p. 139; Schwartz, 1992, 1994; see Table 2b). Schwartz deemed ten values universal-Power, Achievement, Hedonism, Stimulation, SelfDirection, Universalism, Benevolence, Tradition, Conformity, Security (for definitions, see Table 2b). These values derive from three universal requirements of the human condition: needs of individuals as biological organisms; requisites of coordinated social interaction; and survival and welfare needs of groups (Schwartz, 1992, 1994, 2003a; Schwartz \& Bilsky, 1987, 1990).

The ordering of ten human values provides a theory-driven circumplex model, where related values form value clusters in proximity: (1) Self-Enhancement vs Self-Transcendence, and (2) Openness to Change vs Conservation (Schwartz, 1992, 1994, 2003a; Schwartz \& Boehnke, 2004). As two clusters each form opposing pairs, they jointly span a two-dimensional circumplex with (orthogonal) value orientation dimensions as axes, and the difference scores between opposing higher-order value clusters (e.g., Openness-Conservation) yield two dimensions of orthogonal value orientations. Despite the possibility of using finer value distinctions (Schwartz et al., 2012), the ten values suffice to comprehensively represent the clusters and reliably provide the higher-order dimensions (Schwartz, 2003b). 
Dominant cultural values may form the context for developing individual value preferences. Though individuals and cultures differ in their priorities, value measurement functions equivalently across societies (Schwartz, 1994, 2006), and the basic structure of the Schwartz value circle replicates (Bilsky et al., 2010; Schwartz \& Sagiv, 1995). These assets make the four independently measured value clusters and the derived two-dimensional value orientation space (the associated circumplex) suitable candidates for validating character strengths. Comparing strengths to value clusters will be informative, as one can expect people to appreciate the kind of values that correspond to the strengths they possess; likewise, they will tend to possess the exact strengths corresponding to what their value preferences are.

Just like basic human values, the VIA character strengths are cross-culturally inspired (Schwartz, 1992, 1994) and clearly value-laden (e.g., Lavy \& Benish-Weisman, 2021). As character strengths are positively valued, thus related to value-laden personality traits, we posit that they reside at the intersection of two independent personality-relevant frameworks: fundamental personality traits and human values. Consequently, we simultaneously shed light on IPIP-VIA-R's relationships with the Big Five framework and Schwartz's human value clusters and orientations.

Our predictions of dominant associations for the four Schwartz human value clusters were less cogent than for the Big Five domains though (immediate agreement among two raters occurred for slightly more than half of the character strengths). The nature of the value circle made it possible predict a general pattern of inverted signs of correlations for opposite clusters-a positive correlation with Conservation (or Self-Enhancement) implies a negative correlation with Openness-toChange (or Self-Transcendence). Another expectation was that most strengths would likely be positively associated with Self-Transcendence rather than Self-Enhancement, as all character strengths 
share the feature of being socially sanctioned traits. ${ }^{13}$ Overall, for the character strengths scales, one should expect lower correlations with values compared to traits.

Scale Relationships. Prior to analyzing the theoretically expected trait and human value associations, we address one question that has not been convincingly answered for character strengths on the basis of viable strength measures: How are the 24 strengths themselves related to each other? How redundant are they? Would scales be associated similarly across the two countries? We provide a first glimpse at the scale correlations. ${ }^{14}$

${ }^{13}$ From the normative point of view, the strengths should be appreciated as morally good; even if they are not moral strengths in the strict sense, they should form strengths of character that enable success (for society's or the greater good). Yet, people of character cannot simply strive towards individualistic goals, and SelfEnhancement cannot be the driver. Character strengths help overcoming egoism, which is relevant for the evolution and longevity of social organisms. For two strengths (Leadership, Industry/Perseverance) the raters spontaneously predicted a positive association with Self-Enhancement. For Conservation vs. Openness-to-Change, we mostly expected the strengths to scatter along this axis (maybe slightly shifted towards Openness; see SOM_IPIP-VIA-R_RQ6_Nomological-Net-Hypotheses.xIxs). We refrained from forming strong hypotheses about IPIP-VIA-R Spirituality/Religiousness scale, as it blends values related to Self-Transcendence/Self-Enhancement and Openness-to-Change/Conservation. Various constellations of Believing, Bonding, Behaving, or Belonging shape one's religious style or spiritual experiences, and these four aspects are stressed differently across cultural and socio-economic contexts (Gennerich \& Huber, 2006; Saroglou, 2011). Whereas Spirituality may relate to a preference for Openness-to-Change and Self-Transcendence values, Religiousness may relate to Conservation and Self-Enhancement (e.g., Tradition expressed in rites and beliefs; Peterson \& Seligman, 2004; Saroglou et al., 2004; Saucier \& Skrzypińska, 2006; Schwartz \& Huismans, 1995).

${ }^{14}$ To date, the VIA classification is not psychometrically firmly established, and factor-analyzing the 24 scales simultaneously reveals scale redundancy (e.g., Ng et al., 2017). The overlap is related to the strength 


\section{Method}

Participants. Samples 4 and 5 had provided responses to the IPIP-VIA-R survey and to Big Five and human values questions. Despite merely two missing data points in Germany's IPIP-VIA-R (Curiosity, Zest), in both countries Schwartz value items were ("technically") missing more frequently (yet less than 5\%), due to a "don't know" option. This resulted in different case numbers in the (pairwisepresent) bivariate correlation matrix. Validity correlations for higher-order value clusters required working with complete-cases only. A sensitivity check based on SEM-based pseudo-indicator models (PIM; Rose et al, 2019), which accommodates values missing at random in manifest indicators by Full Information Maximum Likelihood (FIML), ruled out biases in the more intuitive approach presented below.

Big Five measurement: BFI-2-S. The BFI-2-S is a 30-item questionnaire available, among many other languages, in English (Soto \& John, 2017b) and German (Danner et al., 2019). It measures the Big Five domains of Extraversion, Agreeableness, Emotional Stability (or Negative Emotionality/Neuroticism), Conscientiousness, and Openness (Open-mindedness). To provide both bandwidth and fidelity, the BFI-2 framework provides for each domain three underlying facets (see Table 2a). The BFI2-S derives from its parent, the 60 -item BFI-2. From there it inherits $50 \%$ of the items while keeping the factor structure robust. The response options are 5-point Likert-type $(1=$ Disagree strongly, $2=$

hierarchy, which exceeds the present scope but receives attention elsewhere (e.g., Partsch et al., 2021). Previous research occasionally found that strengths form a characteristic two-factorial circular structure: a circumplex with the two axes "self-oriented" versus "other-oriented" and "head" versus "heart" (Park et al., 2017; cf. Rosenberg et al., 1968, for intellectual and social desirability being the drivers). "Head" represents intellectual strengths (e.g., Curiosity, Judgment, Love of Learning) and "heart" reflects emotional (interpersonal) strengths (e.g., Forgiveness, Gratitude, Kindness). Close strengths are thus likely to co-occur, distant ones less likely. Yet, in the absence of proper measurement models for previous strength assessment, we refrain here from speculating a priori about the empirical higher-order structure for IPIP-VIA-R. 
Disagree a little, 3 = Neutral; no opinion, 4 = Agree a little, $5=$ Agree strongly). The BFI-2-S has reportedly good reliability and converges very well with the full BFI-2 at the domain level. Thus, we hypothesized about the Big Five at the domain level, operationalized as manifest scale means after properly recoding reverse-keyed items (http://www.colby.edu/psych/wp-content/uploads/sites/50/ 2013/08/bfi2-form.pdf).

For facet and domain scores, we used the authors' aggregation rules. Five domain scores were derived by averaging scored of the balanced sets of six items per domain (Soto \& John, 2017a, 2017b; Danner et al., 2019). Simultaneously, each domain score represents the aggregate of three equally weighted facets. In the BFI-2-S, ARS is controlled at the level of each domain, on which our a priori hypotheses focused, but not at the level of facets.

Basic human values measurement: HVS. We used the Human Value Scale (HVS) taken from the European Social Survey (ESS; Schwartz, 2003a, 2003b). The HVS is based on one of the most common measures of Schwartz values, the Portrait Values Questionnaire (PVQ; Schwartz et al., 2001). The ESS version includes 21 PVQ items, with a few wordings revised to better cover the content of the ten basic values. Each value is represented by two items, except for Universalism (three items). The HVS is available in English and German (Schwartz et al., 2015). Items are short verbal portraits of different people that convey the importance of different values to them, such as "It is important to him to be rich. He wants to have a lot of money and expensive things" (Power). We used the genderspecific versions with different pronouns. For each item, respondents indicate how similar the person described in the item is to them on a 6-point rating scale $(1=$ Very much like $m e, 2=$ Like $m e, 3=$ Somewhat like me, 4 = A little like me, $5=$ Not like me, $6=$ Not like $m e$ at all (plus an alternative response category Don't know). According to the protocol and ESS coding rules for HVS scales (Schwartz, 2003a, 2003b), when analyzing scale correlations, the ratings must be corrected for individuals' mean ratings beforehand. Only then they reflect value priorities free from individual response-style differences, correct for individual differences in the use of the exclusively positivelykeyed response scales, and yield the circumplex with balanced values and polar opposites. 
Individually centered (ipsative) scores express relative value preferences, which is crucial for correlational analyses and to maintain the circumplex structure.

The 21-item ESS-questionnaire is economic in its focus on capturing four value clusters reliably (but not each basic value). We computed four indexes for Self-Enhancement, Self-Transcendence, Conservation, and Openness-to-Change. In line with ESS-prescriptions, higher-order dimension scores are formed from difference scores between value clusters, such that high scores reflect the relative preference of values of Self-Transcendence over Self-Enhancement, and Openness-toChange over Conservation (Schwartz, 2013; https://essedunet.nsd.uib.no/cms/topics/1/).

Analytical approach. We computed Pearson correlations between each of the IPIP-VIA-R scale scores. This allows for comparing our findings with previous ones of similarly analyzed, single- or multiitem strength scales (e.g., McGrath et al., 2020; Ruch et al., 2007; Ruch et al., 2014). For a comparison of IPIP-VIA-R validity coefficients with McGrath et al. (2020), we disattenuated the short scale correlations with the Big Five framework also for unreliability (single and double disattenuation for predictor and criterion variable).

To explore the overlap between each strength and basic personality variables, we also computed regression models that predict each strength by the primary Big Five domain (complemented by all five domains), alternatively by the primary facet (complemented by all 15 facets). We identified the major predictors via associated multiple- $R$ values (and $R^{2}$ as variance explained) to identify which character strengths overlap (rather strongly or weakly) with the pool of all Big Five domains or all 15 facets, respectively. Finally, we related strengths to values not just by correlating each strength with each value cluster but also used cluster difference scores representing the two higher-order value dimensions. Using the correlation coefficients between strengths and higher-order value dimensions as a basis for coordinates (ranging between -1 and +1 ) allows projecting each strength into the two-dimensional space of higher-order value orientations: Self-Transcendence vs. Self-Enhancement (approximating a social growth focus) and Openness-to-Change vs. Conservation (approximating a personal growth focus). 


\section{Results}

Interrelationships among strengths. Character strength scales were almost exclusively positively correlated (see Table 11). That is, they formed a positive manifold. The mean of the average correlation for each IPIP-VIA-R scale with all other scales was $r=.37(.31)$ in the U.K. (Germany). According to each scale's average correlation, in both countries Modesty and Prudence had the lowest overlap with other scales, while the greatest overlap with other scales in both countries had Gratitude, Perspective, Curiosity. The highest correlation coefficients found for any pairs of scales in Germany replicated in the U.K.: Curiosity with Love of Learning (.61 and .58, in Germany and the U.K., respectively), and Zest with Hope (.61 and .61). In the U.K., though, other scales correlated slightly higher: Gratitude with Kindness (.65), and Leadership with Social Intelligence (.64), which also correlated substantially but somewhat weaker in Germany (.54 and .45 , respectively). When scales were unrelated, such as Prudence with Spirituality/Religiousness, this held in both countries (.06); likewise, Self-Regulation with Humor (.16 and .12 in the U.K. and Germany, respectively).

These strength correlations were generally similar across countries. The average difference between correlation coefficients across countries amounted to $0.06(S D=0.07)$. There were only a few strengths for which the differences were larger. For example, Forgiveness correlated significantly with Judgment and Spirituality/Religiousness at $r=.31$ and .38 in the U.K., but in Germany Forgiveness did not correlate significantly with Judgment, $r=.02$, and much lower with Spirituality/Religiousness, $r=.13$. Leadership correlated significantly (and negatively so) with Modesty in Germany, but not so in the U.K. ( $r=-.19$ vs .03).

Relationships with Big Five domains. As evident from Table 12, most character strengths were clearly linked to the (primary) Big Five domain as expected. For 14 scales, both countries confirmed the respective relationship as the strongest one. The strength of associations between IPIP-VIA-R scales and all BFI-2-S domain scores usually fell in the mid-range (.30-.60). Of the many correlations, let us highlight the strongest (absolute) correlation coefficients found between each domain and any strength (for U.K./Germany, respectively): Leadership with Extraversion (.60/.66), Kindness with Agreeableness (.64/.61), Industry/Perseverance with Conscientiousness (.59/.69), Hope with 
Negative Emotionality/Emotional Stability (58./.73.; alternatively Zest: .58/.69), and Originality with Open-Mindedness (.62/.67). These scale correlation coefficients mark the upper boundary of convergent validity coefficients for manifest IPIP-VIA-R strength scales regarding the Big Five domains. All the other strengths also showed substantial convergence with Big Five domains but, at the same time, the coefficients revealed that most strengths cannot just reflect a single (or multiple) Big Five domain(s) in disguise (square, for instance, the adjusted multiple- $R$ values in Table 13 that result from multiple regression models predicting each IPIP-VIA-R scale with all Big Five domains discussed in the next subsection, which hardly cross the threshold of $50 \%$ of explained variance). On average, the scale intercorrelation coefficients amounted to $\bar{r}=.51(S D=0.09)$ and $=.52(S D=0.12)$, for the U.K. and Germany, respectively. For more fine-grained insights, please refer to Table 12.

Our presentation so far highlights the convergent validity for most IPIP-VIA-R strength scales for fundamental personality traits. In those cases where our hypotheses did not fully bear out, that is, where a different domain produced a higher association than the predicted one, the win was usually by a small margin only. For instance, the correlation between Social Intelligence and Agreeableness emerged as envisioned (.45/.39), but Extraversion correlated marginally higher in both countries (.48/.43). The unexpected relationship is plausible in hindsight, given the high level of Sociability found in extraverts. As for another example, Modesty correlated with Extraversion as expected (-.30 in both countries), yet a similar relationship with Agreeableness surfaced in the U.K. (.36) and in Germany (.30). (Note that for these strengths Peterson and Seligman (2004) did not forecast any associations in their Table 3.7 either.) In the case of Humor, the two countries disagreed on the primary domain (.43 for Agreeableness in the U.K.; .58 for Extraversion in Germany). The latter correlation for Germany deviates from our own expectation but confirms Peterson and Seligman's (2004; Table 3.7). Rather than interpreting the pattern as outright disconfirmation or considering such scales as "weak", these associations unveil some difficulty in making clear-cut predictions about primary domains. The one scale we had anticipated to behave unruly in this regard, Spirituality/Religiousness, indeed correlated low and on par with all domains (.17-.36; cf. McGrath et al., 2020). 
Two "real" disconfirmations question our understanding of Equity and Valor/Bravery. Our intuition was to associate Equity primarily with Conscientiousness (.35/.41), as we assumed a motivation to act in a just, trustworthy, and dependable manner in social contexts as the motor behind this association. Yet, both countries agreed that Agreeableness (.63/.61) was the primary associate, thereby highlighting the inner drive to maintain social peace associated with Equity. Similarly, Valor/Bravery appears to require much less the absence of Anxiety or Negative Emotionality $(-.24 /-.28)$ than the expression of Extraversion (.37/.41), probably as Assertiveness in extraverts helps challenge others and prevail over opponents in social encounters.

Nearly all strengths correlated with all Big Five domains. Rarely did a strength not correlate with one of the domains (e.g., Integrity was largely unrelated to Extraversion). The presence of multiple domain associations yields first evidence that strengths reside at intersections of basic traits and cut right through the variable space of personality, though character strengths and the lexical personality space are not redundant (as we will see next).

Conceptual overlap with Big Five domains and facets. To shed light on the redundancy between strengths and basic personality variables, in line with McGrath et al.'s (2020) approach, we regressed each strength on a) the primary domain, b) all domains, c) the primary facet, and d) all facets. Looking at the Big Five facet level allows for a more fine-grained location of the strength in the personality space. The facet-level analysis is informative, because Big Five facets and character strengths are similarly abstract and allow for rather symmetrical abstractness of predictor and criterion variables. Also, using 15 facet scores rather than five domain scores as predictors in regression models yields more statistical power for explaining the variance in strength scales. We additionally corrected correlations for unreliability of the predictor (single disattenuation) and, on top, for the unreliability of the criterion (double disattenuation; see also SOM_IPIP-VIA-R-RQ6_Validity-Correlations-BFI-2-S.xIsx).

Tables 13a and 13b show in descending order, separately for each country, which domains and facets overlapped mostly with strengths. To summarize the picture: At the level of domains, the countries converged on the single domain with the largest correlation for 19 strengths. This number 
increased to 23 when including the domain with the second largest correlation from Table 12 . The one exceptional scale was Perspective, which showed mid-sized correlations regardless of the domain, making the pattern volatile. At the level of facets, the country-specific analyses converged for 15 strengths on the single facet with the largest correlation, which increased to 18 when counting other facets from the same domain as cross-validated. For instance, Hope correlated mostly with Depression in the U.K. and with Anxiety in Germany, but both Big Five facets also belong to the same domain, Negative Emotionality.

Although rare, it could happen that the facet with the strongest correlation with a strength did not come from the domain which produced the strongest correlation. For instance, Appreciation for Beauty and Excellence, which was related primarily to Open-Mindedness, was best predicted in Germany by the Agreeableness-facet Compassion. Conversely, the facet Creative Imagination predicted Humor best, possibly due to a creative playfulness aspect, though Humor's primary domain was not Open-Mindedness, but Agreeableness in the U.K. and Extraversion in Germany.

Interestingly, each strength's strongest facet correlation tended to be of similar size as the correlation with its primary domain. For most strengths slightly higher coefficients resulted (e.g., $r=.66$ vs. .62 and .75 vs. .67 for Originality in the U.K. and Germany, respectively), while for few strengths the opposite held (e.g., . 56 vs. .63 and .57 vs. .61 for Equity). This finding suggests that, from the perspective of each strength, the primary relationship with a Big Five domain is mostly driven by a specific facet that can be identified: For instance, Originality is not just related to Open-Mindedness broadly, but specifically reflects the facet Creative Imagination. This pattern conforms to strengths residing at a lower level than Big Five domains in the nomological network, while being roughly as abstract as personality facets.

As each personality facet was measured with two items, hence with less precision than each domain based on six items, we observed overcorrection for some disattenuated facet correlations that might be mistaken as perfect conceptual overlap. Note that the adjusted- $R$ coefficients from multiple regression are more informative and contradict the notion that strengths are identical with personality facets. Notably, with both IPIP-VIA-R and BFI-2-S controlling for ARS at the scale level, 
none of the strengths could be completely forecast by taking in all Big Five domains (or facets). Squaring the reported multiple- $R$ correlation coefficients from Tables $13 \mathrm{a}$ and $13 \mathrm{~b}$ shows that all Big Five domains (or facets) together hardly ever explained more than $50 \%$ of the variance in strength scales. The comparison of adjusted multiple- $R$ coefficients to zero-order correlations further demonstrates that adding several domains (or multiple facets) hardly improved the statistical predictions (despite facet-level models being statistically powerful).

Some character strengths did overlap notably with single personality facets, but only four (seven) primary facet correlations exceeded unattenuated values of .60 in the U.K. (Germany). Statistical cutoffs can never demarcate if concepts are close to being identical, not even after correction for scale unreliability. Take Love of Learning as an example. Even a disattenuated correlation of .87 with the facet Intellectual Curiosity in the U.K. leaves sufficient headroom for conceptual differences. One can easily envision that a Big Five facet-comprising the BFI-2-S items "being a complex, deep thinker" and "having little interest in abstract ideas" - is associated with Love of Learning, but facet and strength zoom in on different cognitive motivations and processes. And yet, we also observed conceptual closeness between other scales, such as between Compassion-measured with the BFI-2S items "being compassionate, having a soft heart" and "can be cold and uncaring" - and Kindness as a strength. Such concepts may indeed be exchangeable. They require close inspections of scale content before preferring either of the scales.

Relationships with Human Value dimensions. As regards basic human values, the HVS scales showed the typical pattern of reversed correlations across those value clusters that oppose each other and form the ends of the higher-order value dimensions (see Table 12). For instance, when Modesty or Prudence had positive correlations with Conservation, the correlations with Opennessto-Change were negative; other examples are Originality or Humor that showed the reversed pattern of signs. When using the respective difference scores for representing the first higher-order dimension (Self-Transcendence vs. Self-Enhancement), character strengths were predominantly associated with Self-Transcendence rather than Self-Enhancement (with the exception of Leadership; see Figure 3). This pattern supports the social value recognized in, and ascribed to, character strengths, even 
those strengths that are rather intellectual in nature (e.g., Love of Learning). For the orthogonal higher-order dimension, there was no exclusive proximity to one pole. As Table 12 shows, some strengths correlated positively with Openness-to-Change (e.g., Curiosity, Humor, Originality, Valor/Bravery), others with Conservation (e.g., Modesty, Prudence, Self-Regulation), leading to a rather even spread of strengths along the horizontal axis in Figure 3. Likewise of interest are strengths located close to the origin. For instance, Leadership is close to zero on the axis Self-Transcendence vs. Self- Enhancement and thus not associated with a clear value cluster preference, whereas it is clearly associated with Openness-to-Change. Conversely, Judgment and Perspective are as related to Openness-to-Change as they are to Conservation; however, they manifest their potential through transcending rather than enhancing the self. Spirituality/Religiousness showed small or null correlations with the value dimensions.

\section{Discussion}

Taken together, the results in Tables 11-13 reveal four important patterns. First, the pattern of correlations of the character strengths with personality and values showed that, despite noticeable overlap between some scales (for which social desirability may partly account), within the VIA classification the 24 character strengths as measured with IPIP-VIA-R are far from being redundant.

Second, nearly all the strengths shifted markedly towards the Self-Transcendence pole, in line with Peterson and Seligman's (2004) conception of strengths as socially valued traits. From this point of view, being of "good character" means overcoming one's selfish orientations in life. Some strengths closest to the Self-Enhancement pole were also the least moral strengths, but at the same time highly valuable for society's and one's own goals: Leadership, Originality, and Industry/Perseverance.

Third, regarding the question of VIA-Big Five redundancy, the answer needs to be differentiated. Each strength was meaningfully related to at least one Big Five personality domain, in most cases to the ones we predicted, and in most cases to all the Big Five domains (although to varying degrees). While this pattern overall supports the IPIP-VIA-R's breadth and nomological network, the multiple associations show that character strengths are interstitial constructs that occupy the 
intersections of several personality traits. In most cases, we were able to single out one primary personality facet to which each strength was most strongly related, too. At times, the content of these personality facets corresponded closely to the content of the strength scales, sometimes even with the labels being close themselves (compare VIA's Originality to Creative Imagination or VIA's Industry to Conscientiousness's Productiveness facet). Other strengths (e.g., Prudence and Spirituality/Religiousness) hardly overlapped with Big Five personality domains or facets at all. Apart from these farthest ends of the spectrum of overlap, most strengths correlated (sans disattenuation) with the Big Five framework between $r=.40$ and .60 .

McGrath and colleagues (2020) reasoned that uncorrected correlations above .60 might serve as evidence for likely scale redundancy, whereas lower correlations of .50 would rather reflect closely related but distinct constructs. If we set the findings for Germany as the reference and apply these criteria to our study, seven character strength scales showed strong overlap with Big Five domains: Kindness, Originality, Leadership, Equity, Hope, Zest, and Industry/Perseverance. At the proper level of abstraction, strong overlap with facets emerged virtually for the same strengths, with Integrity replacing Equity in the list. All in all, most character strengths are not redundant to Big Five domains or facets. They complement the set of personality-related concepts that have been omitted in the lexical tradition, which focused on person descriptors not subject to moral evaluation. With confidence, we virtually draw the same conclusion as others, but base our judgment on a set of content-valid and cross-culturally invariant IPIP-VIA-R short scales: "16 of 24 strength scales were not fully accounted for by personality facets" (McGrath et al., 2020, p. 132).

Finally, the correlations coefficients were remarkably similar across the countries, confirming the robustness of the nomological net and the quality of the translation and adaptation achieved. For instance, the average Euclidean distance between U.K. and German coordinates in the Schwartz value space (see Figure $3 \mathrm{c}$ ) amounted to 0.11 ; that is, across both axes, the two countries' correlation coefficients deviated hardly at all (or only by 0.11 points on average). Together with the encouraging results about scale reliability (see RQ3), the overall similar pattern of scale intercorrelations suggests that the IPIP-VIA-R scales work similarly in Germany and the U.K. 
In sum, refining the IPIP-VIA measures yielded short scales with clear, value-laden, and theoryconforming validity patterns. IPIP-VIA-R poses a valid window into traits that emerged from, and conform to, a cross-cultural perspective. Yet, character strengths complement our view of the personality variable space and look at it from a different angle. Researchers may differ in their preferences for using a theoretically derived, cross-cultural measurement approach (character strengths) or an inductive approach that reflects lexical parsimony (Big Five framework). The next section addresses the utility of these scales by inspecting criterion validity.

\section{Research Question 7:}

\section{Is the Criterion Validity of IPIP-VIA-R Scales Sufficient?}

Having established the position of the IPIP-VIA-R in a nomological net with personality traits and basic human values, we next tested the scales' criterion validity. Criterion validity is one of the cornerstones of a scale's practical utility. The idea that character strengths promote "the good life" with an optimal balance between pursuing individual life goals and meeting social demands is foundational to the VIA model (Peterson \& Seligman, 2004; Seligman, 2002, 2011). Therefore, life satisfaction is a key outcome of which the character strengths should be predictive. In addition to this subjective indicator, we also probed the criterion validity of character strengths for a more factual life outcome: health. Even though we had to rely on self-reports, including health provides a more complete picture of the predictive utility of the IPIP-VIA-R scales for indicators of "the good life." Keep in mind, though, that according to their theoretical conception each character strength is essentially "[...] morally valued in its own right, even in the absence of obvious beneficial outcomes" (Peterson \& Seligman, 2004, p.19, emphasis added).

Previous research suggests that positive correlations exist between all character strengths and life satisfaction (Ruch et al., 2007), with the strongest correlations emerging for the transcendence strengths Hope, Gratitude, and Spirituality/Religiousness, furthermore with the strengths Capacity for Love, Curiosity, and Zest (Park et al., 2004; Proyer et al., 2011; Weber et al., 2013). As alluded to above, we expected positive, albeit somewhat weaker, associations for some strength scales with 
health. Proyer et al. (2013) found significant positive correlations between a single-item health selfrating and the strengths Hope, Humor, Industry/Perseverance, Self-Regulation, and Zest.

Furthermore, we provide a first exploratory overview of the incremental validity above and beyond the Big Five domains of the 24 IPIP-VIA-R scales for life satisfaction and health. We report incremental validity beyond Big Five domains, because the IPIP-VIA-R scales might often be applied next to short scales assessing the Big Five domains rather than the facets (e.g., in large-scale assessment). At the same time, strengths and facets reside on a more comparable level of abstraction or aggregation in the hierarchy of constructs (i.e., the IPIP-VIA-R strengths are conceptually roughly as broad as the Big Five facets) and the number of predictors multiplies in a facet-based analysis. Testing incremental validity of the character strengths beyond the Big Five facets thus represents a stricter approach. While we focus on domain-based incremental validity, we also run facet-based analyses as a cross-validation for domain-based findings (see SOM_IPIP-VIA-R_RQ7_Incremental-Validity.pdf).

Gender. We computed the IPIP-VIA-R correlations with gender, too. Gender may be differentially associated with character strengths. However, the character strengths framework does not provide firm clues for specific hypotheses. Associations between some strengths and gender seem intuitive (e.g., one might expect females to score higher on Kindness; Ruch et al., 2007). Yet, any emerging correlation coefficients need to be interpreted with caution as their origin would be unclear. Large gender difference would alert us to reinspect the criterion validity of strengths only after controlling for gender as a covariate in regression models.

\section{Method}

We conducted all analyses in both Germany and the U.K. using Samples 4 and 5. Previous research had regressed life satisfaction on all strengths in a single step in regression analysis and found that more than $40 \%$ of variance overlapped with the criterion (Noftle et al., 2011). We followed this approach but, for a more nuanced view, first investigated each strength's association in separate models. To this end, we measured the general and current satisfaction with life (L-1 scale; Nießen et al., 2020): “All things considered, how satisfied are you with your life these days?” with 11 graded response options ranging from not at all satisfied (1) to completely satisfied (11). The L-1 scale 
converges highly with the late Diener's SWLS scale (Diener et al., 1985). Health status was measured health with an ad hoc single-item reading "How is your health in general? Would you say it is ..." on a 5-point response scale from very good (1) to very bad (5).

We based criterion validity for the three outcomes on bivariate (Pearson) correlations. For analyzing incremental validity beyond the Big Five domains, we conducted multiple-regression analyses like those by Noftle and colleagues (2011). In a baseline model, we first regressed the criterion on all Big Five scores (i.e., five domains or 15 facets). We then added one character strength to the model and judged its incremental validity by its associated $\Delta R^{2}$ and standardized regression coefficient. Similar to Noftle and colleagues (2011), we also provide the criterion variance explained by each strength $\left(r^{2}\right)$ when regressing the outcome variables exclusively on that strength. Using personality-specific, meta-analytically derived guidelines (Gignac \& Szodorai, 2016), we interpreted $r=.10, .20$. and .30 as small, medium, and large effect sizes, respectively. Accordingly, we interpreted $\Delta R^{2}=1 \%$ as small, $\Delta R^{2}=4 \%$ as medium, and $\Delta R^{2}=9 \%$ as large $R^{2}$-change.

\section{Results}

We begin by inspecting gender effects. Then we present results on criterion validity of the 24 IPIP-VIA-R scales in Table 14 and incremental validity in Table 15, respectively (for further details, see also Table D1 in SOM_IPIP-VIA-R_RQ7_Incremental-Validity.pdf).

Gender. For both countries, we mostly found negligible gender differences, though significant positive correlations of small size to medium size emerged with Spirituality/Religiousness, Kindness, Gratitude, Appreciation of Beauty and Excellence, Integrity, Equity, and Capacity for Love (in descending order). In Germany, Prudence too correlated with gender. Positive correlations indicate that a strength is more pronounced in women. This pattern of correlations is plausible and indicates that, for a few scales, direct comparisons across gender groups may profit from using gender-specific norms. Yet, strongly biased criterion correlations are unlikely. The effect sizes we found are in line with the maximum gender differences observed for the BFI-2 domains (e.g., $r_{\max }=.17 / .26$ for Agreeableness in Internet/Student samples; Soto \& John, 2017a) and below the meta-analytically derived maximum effect size for Big Five facets (e.g., $\bar{r}_{\max }=.24$ for Assertiveness; Feingold, 1994). 
Criterion validity: Life satisfaction. In line with theory, among the three criteria, life satisfaction showed the highest average correlation with character strengths $\left(\bar{r}_{\mathrm{UK}}=.23, \bar{r}_{\mathrm{DE}}=.27\right)$. In line with previous findings, large correlations were found with Hope, Gratitude, Spirituality/Religiousness, Capacity for Love, Curiosity, and Zest either in both the U.K. and Germany or in at least one of the countries. In addition, across both countries, life satisfaction had large correlations with Industry/Perseverance. In Germany, additional five correlations were of large magnitude. All other correlations were positive and ranged between small and medium/large size. The only exceptions were the correlations of life satisfaction with Modesty (non-significant in Germany, and negative in the U.K.) and Prudence (statistically non-significant in both countries). From a theoretical point of view suggesting that all character strengths should somehow contribute to a happy life, the findings for the latter scales are somewhat unexpected, yet not completely implausible. Modesty may be a "mixed blessing" in that it may prevent return of investments in social or economic respects. Also, people low on Modesty might want something special out of life and might consider themselves as satisfied as highly modest people. People high on Prudence might hesitate to evaluate their lives as satisfying, either because they focus on risks, undermining their satisfaction, or because life has been rife with disappointments, making them cautious. Also, cultures may differ on how much importance is given to personal happiness as a criterion for a "good life." Modest and prudent people may be orienting their lives more toward something other than self-gratification.

In both countries, Capacity for Love, Industry/Perseverance, Spirituality/Religiousness, and Zest showed positive incremental validity for life satisfaction above and beyond the Big Five domains. Also, Curiosity, Gratitude, and Hope, which appeared as strongest correlates of life satisfaction in previous and present studies, showed incremental validity beyond the Big Five in at least one of the countries. The increment for Self-Regulation had a negative beta-weight in both countries, indicating that the positive bivariate correlations were generated by parts that overlapped with the Big Five, and the unique part predicted life satisfaction negatively, potentially indicating affective-behavioral over-controlling. Other incremental effects varied across countries (see Table 15). Among the 
significant incremental effects, the $R^{2}$-change ranged between $0.4-5 \%$ in the U.K. and $1-7 \%$ in Germany, that is, between small and medium to large size.

In the facet-based cross-validation-which are based on powerful three times the number of predictors in the baseline model-the 10 significant incremental effects for predicting life satisfaction in the domain-based model in Germany replicated. In the U.K., for life-satisfaction six out of eight increments beyond domain scores replicated with facets. Effect sizes were smaller on average, but still substantial, $R^{2}$-changes of $0.4-5 \%$ for life satisfaction (for details, see SOM_IPIP-VIA-R_RQ7_Incremental-Validity.pdf).

Criterion validity: Health. The average correlation between the 24 IPIP-VIA-R scales and health varied across the two countries: Whereas in Germany most correlations ranged between .10-.30, in the U.K. more than half of the correlations were non-significant. In line with Proyer and colleagues (2013), we identified Hope, Humor, Industry/Perseverance, Self-Regulation, and Zest as significant correlates of health across both countries: Hope, Humor, Industry/Perseverance, and Self-Regulation were among the scales with small to medium-sized (significant) correlations in UK and produced mostly mid-size correlations in Germany. In both countries, Zest even showed a large correlation. Noteworthy, Curiosity was also a large positive correlate in Germany. Prudence showed a significant negative correlation with health in Germany, as did Modesty in both countries. This finding is compatible with the view that Prudence and Modesty may not be universally beneficial, although the cross-sectional data cannot rule out that bad health may conduce to the development of both "strengths", Prudence and Modesty, or that these strengths are sometimes formed as a result of negative life experiences that restrict life satisfaction and health, which in turn may cloud the otherwise positive contribution of these strengths to life satisfaction and health.

Given that many character strengths were uncorrelated with health, incremental validity of IPIP-VIA-R scales beyond the Big Five was limited. Also note that even all the Big Five taken together hardly explained large amounts of criterion variance $(<8 \%)$. On the positive side, Zest possessed incremental validity in both countries, despite its strong overlap with Neuroticism (and the BFI-2-S'S Depression facet in particular). Furthermore, in Germany, about one quarter of the IPIP-VIA-R scales 
showed both significant positive correlations with health and positive incremental validity. Modesty not only correlated negatively in Germany, but also had a negative beta-weight. Though this finding was not corroborated cross-culturally, it leaves open the possibility that unique parts in Modesty that do not overlap with the Big Five are rather detrimental to health, undermining a strict interpretation of this trait as character strength. Unfortunately, most incremental effects found in UK cannot be interpreted meaningfully (content-wise), because the bivariate correlations were already non-significant (and negative beta-weights are likely due to statistical suppression). With $R^{2}$-change ranging between 1-3\%, the incremental effects in Germany were small (or medium at best), as was the incremental effect of Zest in the U.K. (2\%).

In the facet-based analysis in Germany, seven out of nine incremental effects mirrored the findings from the domain-based analyses (1\%). In contrast, the only well-interpretable effect predicting health beyond domain scores in the U.K., Zest, did not replicate with facets.

\section{Discussion}

Our findings corroborated the criterion validity of the VIA-IPIP-R scales for life satisfaction and health. On average, we replicated across countries large and medium positive associations of strengths with life satisfaction and health, respectively. More (and descriptively stronger) positive correlations across the criteria emerged in Germany than in the U.K. Rather than speculating about stronger predictive validity in a German context, we attribute the differences to better data sample quality (for which we have independent indications such as unit-nonresponse) and the favorable German translation/adaptation. At any rate, the robust and often large correlations of the character strengths with the criteria demonstrate that the IPIP-VIA-R scales not only have good structural validity (see RQ2-4) but also predictive utility. This is especially true for life satisfaction - the outcome with which strengths should be associated if they indeed promote "the good life", as stipulated by the VIA framework (Peterson \& Seligman, 2004), even if each character strength is (morally) valued in its own right (Stahlmann \& Ruch, 2020).

We also found some evidence that IPIP-VIA-R has incremental validity above and beyond the Big Five domains. Because there is a clear conceptual overlap between the VIA framework, Big Five 
domains and especially some facets (see RQ6 and Table 13), our analyses constitute a very conservative test of the character strengths' incremental predictive validity. Nonetheless, several strengths showed incremental validity for these criteria beyond the Big Five domains (Table 15). Life satisfaction emerged as the criterion associated most strongly with character strengths beyond the Big Five, whereas health was harder to predict incrementally and overall.

Correspondingly, some of the resulting effect sizes were medium to large for the criterion life satisfaction, though smaller for health. We interpret the incremental value of character strengths $\left(R^{2}-\right.$ change) against the background of the baseline models $\left(R^{2}\right)$ in which the Big Five domains were the only predictor variables: About $30 \%$ and $15 \%$ of criterion variance were explained by the Big Five domains. Adding character strengths explained $4-7 \%$ more of the variance at most, which we consider substantial, given that a single character strength then has about the same influence as has the average Big Five domain. In Germany, most incremental effects replicated when using multiple Big Five facets instead of domain scores. Unfortunately, the same cannot be said to apply to the U.K., which might be attributed to the alleged differences in sample quality and improved item wordings in the German adaptation of IPIP-VIA-R items.

In sum, our results demonstrate the utility of IPIP-VIA-R for predicting important life outcomes-sometimes over and above the Big Five framework, regardless whether using domain or facet scores.

\section{General Discussion}

About two decades ago, Peterson and Seligman (2004) presented a seminal classification of character strengths that has led to a renaissance as a research topic of "human character"-a concept that has interested philosophers for millennia but that had long been abandoned in personality psychology in its fundamental search for personality characteristics that exist from social evaluation. In contrast to the lexical tradition in which evaluative (including moral) adjectives were deliberately, though only partially, removed from personality item pools, Peterson and Seligman's (2004) VIA framework is based on a comprehensive theoretical analysis of personal qualities that they deemed (morally) "valued in their own right" across cultures and that they thought promoted individual and 
collective well-being, or in short: the "good life." Owing to its firm roots in cross-cultural research, the VIA framework can provide a valuable complement to the Big Five or HEXACO models for individual differences research. It is therefore not surprising that researchers from different disciplines have embraced the VIA framework and produced a rapidly growing body of evidence on the precursors and outcomes of character strengths.

However, as our review showed, there are abiding and unresolved limitations with the most widely used VIA measurement instruments that threaten the validity of extant research on character strengths. In the present study, we therefore reported on a major research project through which we aimed to advance the assessment of human character by developing short, balanced-keyed, contentvalid and cross-culturally applicable scales for the 24 VIA character strengths based on the open-access IPIP.

Based on the findings presented, we can now answer the seven research questions that guided our project. (1) As we suspected, and as previous research has pointed out for VIA-IS items (Ng et al., 2017), the original 252 IPIP-VIA items do not allow for unidimensional assessment of each character strength. Moreover, as the often small loadings showed, many items have little in common with the core of their target strength, meaning that they lack validity. (2) Fortunately, however, the IPIP does comprise enough content-valid, cross-culturally applicable, and well-worded items so that balancedkeyed short scales comprising four items per strength can be constructed. The resulting scales largely preserve the substantive breadth of character strength definitions but are nonetheless approximately unidimensional. Remaining deviations from strict unidimensionality are likely due to ARS, which constitutes an additional, content-unrelated source of variance.

(3) The shortage of a measurement model generally applicable to the 24 character strengths is over. Single-factor CFA measurement models for each of the 24 character strength scales that control for acquiescent responding show good fit and mostly satisfactory factor loadings. For three scales-Hope, Leadership, and Spirituality/Religiousness-their complex content may require refinement in the long run to minimize correlated residuals that deviate from essential unidimensionality. (4) As to whether the IPIP-VIA-R short scales measure reliably, the answer is a qualified yes. For 
applied scale use, we recommend omega from adequately specified measurement models with ARScontrol, alternatively retest-reliability for manifest scale scores. While for individual diagnostics the scales might have to be amended with more (content-valid) items, the reliability figures were sufficient for the purpose of social surveys. Random measurement error does not bias the (unstandardized) regression coefficients when a scale is used as an outcome in such a survey (Lechner et al., 2021). When character strengths are used as predictors of life outcomes, measurement error can be controlled by using latent measurement models (such as the one presented in RQ3).

(5) Across two languages, all 24 scales performed at the level metric invariance (except for Gratitude where a sole item was found to be non-invariant). This pattern speaks to the same use of measurement units and psychological meaning of strength items, which is a requirement for crossgroup comparisons of factor variances, covariances, and validity correlations (or regression approaches). Moreover, all scales even reached (at least partial) scalar invariance. Scalar invariance is a requirement for comparing means of strength scales across countries. Scalar invariance is often a hard-to-reach goal in cross-cultural research. In view of this, the high degree of measurement invariance the IPIP-VIA-R scales reached is very encouraging and is testament to the success of the rigorous item selection and translation/adaptation process of our project, in which cross-cultural applicability was one of the guideposts from the outset.

(6) Analyses of nomological nets showed that the 24 character strength scales had theoretically plausible associations with both personality domains and facets and basic human values and supported both the convergent and discriminant validity of the character strengths. Analyses suggested that character strengths show some overlap with both personality traits and values, which makes sense because character strengths are morally valued personal qualities. Almost all character strengths reflected Openness-to-change and Self-Transcendence values as judged by their correlations with higher-order value orientations (Schwartz, 1994, 2003a), supporting the claim that these strengths are not primarily in the service of individual self-enhancement but communal in nature. Few character strengths were so strongly related to (sometimes even similarly named) personality facets that the constructs must be deemed identical; this is not surprising, given that character 
strengths partly use the same person-descriptive adjectives as the lexical tradition. At the same time, there were several strengths (e.g., Spirituality/Religiousness, Prudence) that had no conceptual counterpart in Big Five personality domains or their underlying facets at all, and most strengths had substantial unique portions of variance that they did not share with personality traits and basic human values. Thus, character strengths occupy their own position in the trait space spanned by personality traits and values.

(7) We found initial evidence in support of criterion validity. Specifically, the IPIP-VIA-R character strength scales predict life satisfaction and health. Even evidence for incremental validity beyond the Big Five, albeit small, emerged. The verdict is a bit more optimistic for the German than the English scales due to the quality of wordings achieved. Objective indicators are needed to corroborate these findings.

Our findings show that it is possible to measure character strengths as conceived in the VIA framework with IPIP. We are confident that IPIP-VIA-R currently represents the best solution for each character strength that can be obtained within the IPIP alone. The IPIP-VIA-R is a major advancement over previous VIA instruments in that it is explicitly designed with content-validity and cross-cultural applicability in mind, in line with the cross-cultural theoretical underpinnings of the VIA framework. Our findings suggest that the joint rational-empirical approach to deriving brief balanced IPIP-VIA-R scales was successful and yielded efficient, reliable, and valid strength measures.

\section{Limitations and Future Directions for Character Assessment}

Despite the improvements over other character inventories based on VIA, and especially the original IPIP-VIA, IPIP-VIA-R is not perfect. In this regard, our self-imposed constraint to use exclusively items from IPIP has some drawbacks. Although it was our pre-set goal to measure each character strengths with four items (similar to, for example, the BFI-2 facet scales; Soto \& John, 2017a), it was sometimes challenging to select even four items from IPIP-VIA that met all our criteria of content-validity, item wording, and cross-cultural applicability (see RQ2). Occasionally, we had to resort to IPIP items from other sections than IPIP-VIA to meet all demands. Although four items are sufficient for research purposes - which is what IPIP-VIA-R is designed for-the present IPIP-VIA-R 
inventory is not sufficiently reliable for individual diagnostics, and IPIP does not provide enough valid items to construct longer IPIP-VIA scales that would be suitable for individual character assessment. Moreover, the wording of some IPIP items is less than optimal. Items were invented, often by local project volunteers, mostly from the United States. Also, the IPIP limits the options for finding suitable items representing unique aspects of VIA character strengths, because content substantially related to the Big Five core is heavily represented, despite later attempts to shape the IPIP item set somewhat into the direction of content less related to the Big Five (Saucier \& Goldberg, 1998). Yet, by rationally evaluating item content according to strength relevance and wording clarity, we promoted focus and fidelity of each strength scale. Minimizing the influence of ARS, we also balanced bandwidth and fidelity as much as the IPIP framework allowed without inflating reliability by redundant or multifactorial item sets, but by focusing on the common core of each strength.

Note that we only investigated two Western countries and languages. Developing IPIP-VIA-R in parallel in two languages was a major advantage and proved fruitful because we considered crosscultural applicability and translatability already during item selection (see RQ2). Future translations of IPIP-VIA-R are needed for surveys that transcend cultural settings beyond the Indo-Germanic languages we investigated. We believe that the IPIP-VIA-R provides a better starting point for future translations than any prior VIA instrument and would recommend the German over the English version as a source because, as noted in RQ2, we slightly improved the wording of the German translation during the adaption process.

We hope that the IPIP-VIA-R short scales provide an apt measurement basis for future studies, enabling economic assessment in large-scale surveys and longitudinal follow-up. We reiterate desiderata from a decade ago (Noftle and colleagues, 2011). Future research needs to address open questions about the validity of the VIA framework and, to this end, pursue long-term real-world outcomes. Longitudinal studies that examine character development over extended periods of time are required. Research that tackles the validity of VIA character strengths, also with the help of longitudinal designs, has gained new momentum recently (e.g., Gander et al., 2020; Stahlmann \& Ruch, 2020; Wagner et al., 2020). 
Future investigations might also focus on comparative predictive capacity with other instruments assessing strengths. The IPIP advocates the idea of comparative-validity studies that compare original personality inventories for which IPIP scales have been developed with their IPIP counterpart (https://ipip.ori.org/newResearchOpportunities.htm). With IPIP-VIA-R strength scales being more in line with a cross-culturally comparable unidimensional measurement approach than any version preceding it, the exploration of the hierarchical structure among strength scales has become a worthy endeavor.

\section{Conclusion}

Based on a rigorous psychometric evaluation, we recommend the use of IPIP-VIA-R as a basis for future research on character strengths. We believe it works better than existing instrumentsand it is free. 


\section{Author Note}

\section{Corresponding Author}

Matthias Bluemke, GESIS, B6/4-5, 68159 Mannheim, Germany; e-mail: matthias.bluemke@gesis.org

\section{Competing Interests}

We have no competing interests to disclose.

\section{Authors' Contributions}

Conceptualization: MB (E), MP (E), GS (S), CL (E)

Data curation: $M B(E), M P(E), C L(S)$

Formal analysis: $M B(L), M P(E), C L(E)$

Investigation: $\mathrm{MB}(\mathrm{E}), \mathrm{MP}(\mathrm{L}), \mathrm{CL}(\mathrm{E})$

Methodology: MB (E), MP (E), GS (S), CL (L)

Project administration: $\mathrm{CL}(\mathrm{L})$

Resources: GS (S), CL (L)

Supervision: GS (E), CL (L)

Validation: $M B(E), M P(E), C L(S)$

Visualization: $\mathrm{MB}(\mathrm{L}), \mathrm{CL}(\mathrm{S})$

Writing-original draft: $M B(E), M P(E)$

Writing - review and editing: CL (L), GS (S)

\section{Availability of Data and Materials}

Supplemental Online Material (SOM) can be found at OSF (view-only link for review):

https://osf.io/3mfyw/?view only=529e048441414faa9b39a3ed6880123c

The SOM contains more extensive tables and documents on seven research questions (labeled RQ1 to RQ7 in the main manuscript), though it should be possible to evaluate the content of the main 
manuscript without the help of the SOM. Yet, all material is transparently accessible to those interested in further background information or to those who seek additional information about findings on samples other than the ones highlighted or presented exclusively in the manuscript. This is a list with the relevant files of the SOM:

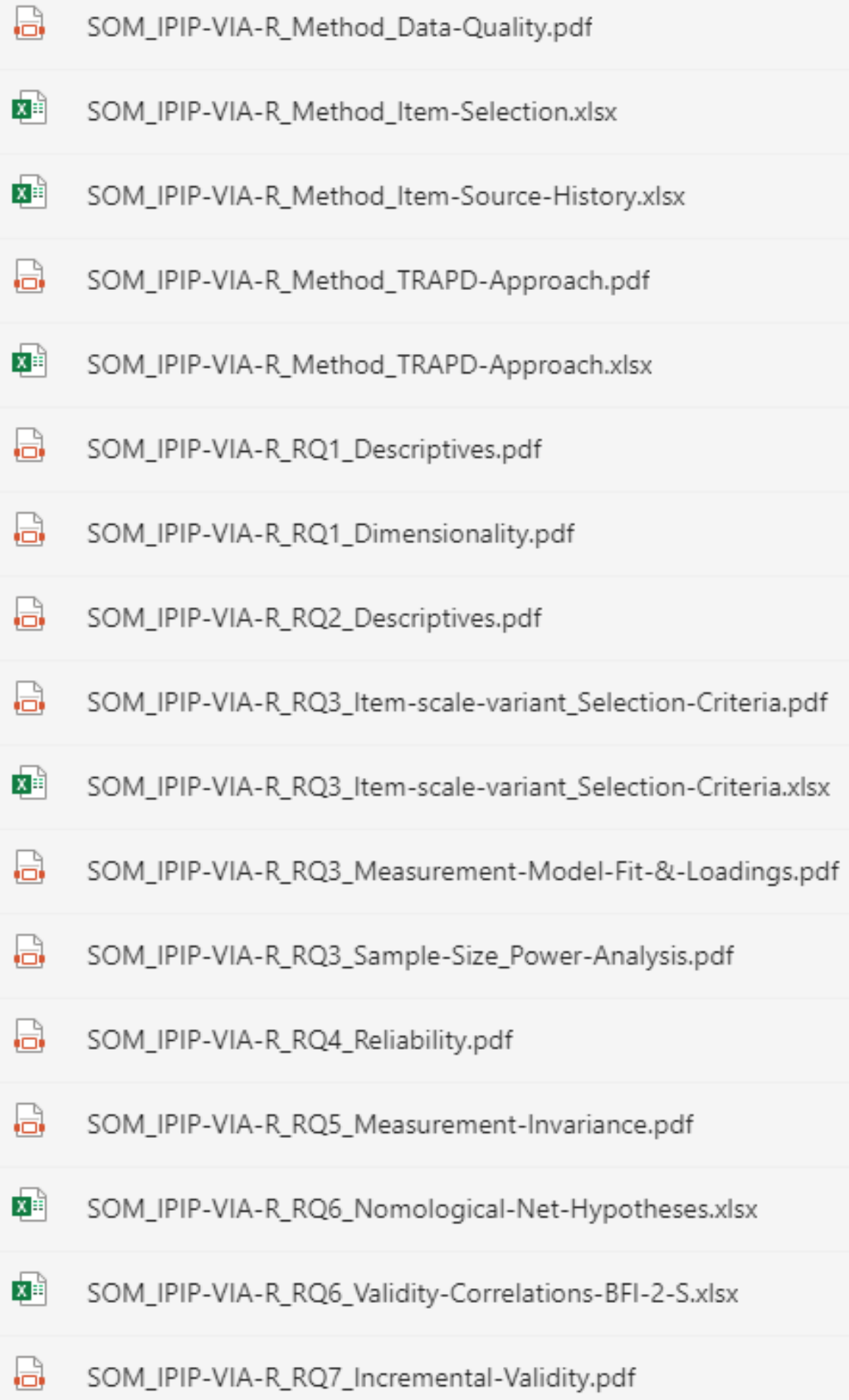




\section{Acknowledgments}

We would like to thank

- Dorothée Behr for serving as an expert translator, mediator and adjudicator;

- Klara Dentler for preparing psychometric properties of the IPIP-VIA-R scales (FACTOR);

- Katharina Groskurth for programming and conducting the web studies, data cleansing (SPSS), references (Endnote);

- Michael Grosz for providing an R-code template for extracting statistical indices (FACTOR);

- IPIP group for collecting and providing IPIP data (ESCS);

- Johanna Klein for preparing psychometric properties of the IPIP-VIA-R scale (FACTOR);

- Verena Kneissle for data preparation (SPSS);

- Thomas Knopf for statistical support for comparing validity coefficients (STATA);

- Julia Krasnoff for support for item review process and documentation of item translations;

- Miriam Pfister for references, data preparation, data cleansing, and documentation of results;

- Beatrice Rammstedt (GESIS/Department Survey Design and Methodology) for financial support (for participant remuneration and for guest researcher stays). 


\section{References}

Ackerman, C. E., Warren, M. A., \& Donaldson, S. I. (2018). Scaling the heights of positive psychology: A systematic review of measurement scales. International Journal of Well-Being, 8(2), 1-21. https://doi.org/10.5502/ijw.v8i2.734

Adelnia, A., \& Vahid Dastjerdi, H. (2011). Translation of idioms: A hard task for the translator. Theory and Practice in Language Studies, 1(7), 879-883. https://doi.org/10.4304/tpls.1.7.879-883

Aichholzer, J. (2014). Random intercept EFA of personality scales. Journal of Research in Personality, 53, 1-4. https://doi.org/10.1016/j.jrp.2014.07.001

Aichholzer, J. (2015). Controlling acquiescence bias in measurement invariance tests. Psihologija, 48(4), 409-429. https://doi.org/10.2298/PSI1504409A

Allalouf, A., Hambleton, R. K., \& Sireci, S. G. (1999). Identifying the causes of DIF in translated verbal items. Journal of Educational Measurement, 36, 185-198. https://doi.org/10.1111/j.17453984.1999.tb00553.x

Allport, G. W. (1927). Concepts of trait and personality. Psychological Bulletin, 24(5), 284-293. https://doi.org/10.1037/h0073629

American Psychiatric Association. (1994). Diagnostic and statistical manual of mental disorders (4 ${ }^{\text {th }}$ ed.). Washington, DC: American Psychiatric Association.

Ashton, M. C. (2005, January). How the IPIP benefits personality research and education. In L. R. Goldberg (Chair), The international personality item pool and the future of public-domain personality measures. Presidential symposium at the $6^{\text {th }}$ annual meeting of the Association for Research in Personality (ARP), New Orleans.

Ashton, M., \& Lee, K. (2007). Empirical, theoretical, and practical advantages of the HEXACO model of personality structure. Personality and Social Psychology Review, 11, 150 - 166. https://doi.org/10.1177/1088868306294907

Ashton, M. C., \& Lee, K. (2020). Objections to the HEXACO model of personality structure-and why those objections fail. European Journal of Personality, 34(4), 492-510.

https://doi.org/10.1002/per.2242 
Ashton, M. C., Lee, K., Perugini, M., Szarota, P., de Vries, R. E., Blas, L. D., Boies, K., \& De Raad, B. (2004). A six-factor structure of personality-descriptive adjectives: Solutions from psycholexical studies in seven languages. Journal of Personality and Social Psychology, 86(2), 356-366. https://doi.org/10.1037/0022-3514.86.2.356

Asparouhov, T., \& Muthén, B.O. (2018). SRMR in Mplus. http://www.statmodel.com/download/SRMR2.pdf

Bagozzi, R. P. (1993). Assessing construct validity in personality research: Applications to measures of self-esteem. Journal of Research in Personality, 27(1), 49-87. https://doi.org/10.1006/jrpe.1993.1005

Beauducel, A. (2011). Indeterminacy of factor score estimates in slightly misspecified confirmatory factor models. Journal of Modern Applied Statistical Methods, 10(2), 583-598. https://doi.org/10.22237/jmasm/1320120900

Beauducel, A., \& Leue, A. (2014). Testing the assumption of uncorrelated errors for short scales by means of structural equation modeling. Journal of Individual Differences, 34(4), 201-211. https://doi.org/10.1027/1614-0001/a000135

Béguin, C., \& Hulliger, B. (2004). Multivariate outlier detection in incomplete survey data: The epidemic algorithm and transformed rank correlations. Journal of the Royal Statistical Society, A 167 (Part 2.), 275-294. https://doi.org/10.1046/j.1467-985x.2003.00753.x

Behr, D., \& Zabal, A. (2020). Documenting survey translation. Mannheim, GESIS - Leibniz-Institute for the Social Sciences (GESIS - Survey Guidelines). https://doi.org/10.15465/gesis-sg_en_035 Bentler, P. M. (1990). Comparative fit indexes in structural models. Psychological Bulletin, 107, 238-246. https://doi.org/10.1037/0033-2909.107.2.238

Bentler, P. M. (2007). On tests and indices for evaluating structural models. Personality and Individual Differences, 42(5), 825-829. https://doi.org/10.1016/j.paid.2006.09.024

Bentler, P. M., \& Bonett, D. G. (1980). Significance tests and goodness of fit in the analysis of covariance structures. Psychological Bulletin, 88(3), 588-606. https://doi.org/10.1037/0033- 
Berry, J. W. (1969). On cross-cultural comparability. International Journal of Psychology, 4(2), 119128. https://doi.org/10.1080/00207596908247261

Berry, J. W. (1999). Emics and etics: A symbiotic conception. Culture and Psychology, 5(2), 165-171. https://doi.org/10.1177/1354067X9952004

Billiet, J., \& McClendon, M. J. (2000). Modeling acquiescence in measurement models for two balanced sets of items. Structural Equation Modeling, 7(4), 608-628. https://doi.org/10.1207/S15328007SEM0704_5

Bilsky, W., Janik, M., \& Schwartz, S. H. (2010). The structural organization of human values: Evidence from three rounds of the European Social Survey (ESS). Journal of Cross-Cultural Psychology, 42(5), 759-776. https://doi.org/10.1177/0022022110362757

Biswas-Diener, R. (2006). From the equator to the North Pole: A study of character strengths. Journal of Happiness Studies, 7(3), 293-310. https://doi.org/10.1007/s10902-005-3646-8

Boateng, G. O., Neilands, T. B., Frongillo, E. A., Melgar-Quiñonez, H. R., \& Young, S. L. (2018). Best practices for developing and validating scales for health, social, and behavioral research: A primer. Frontiers in Public Health, 6, 149. https://doi.org/10.3389/fpubh.2018.00149

Bond, T. G., \& Fox, C. M. (2015). Applying the Rasch model: Fundamental measurement in the human sciences (3rd ed.). Mahwah, NJ L. Erlbaum.

Brosseau-Liard, P. E., \& Savalei, V. (2014). Adjusting relative fit indices for nonnormality. Multivariate Behavioral Research, 49(5), 460-470. https://doi.org/10.1080/00273171.2014.933697

Brosseau-Liard, P. E., Savalei, V., \& Li, L. (2012). An investigation of the sample performance of two non-normality corrections for RMSEA. Multivariate Behavioral Research, 47(6), 904-930. https://doi.org/10.1080/00273171.2012.715252

Brown, T. A. (2015). Confirmatory factor analysis for applied research $\left(2^{\text {nd }}\right.$ ed.). New York, NY: Guilford.

Browne, M. W., \& Cudeck, R. (1993). Alternative ways of assessing model fit. In K. A. Bollen \& J. S. Long (Eds.), Testing structural equation models (pp. 136-162). Newbury Park, CA: Sage. 
Buja, A., \& Eyuboglu, N. (1992). Remarks on parallel analysis. Multivariate Behavioral Research, 27(4), 509-540. https://doi.org/10.1207/s15327906mbr2704_2

Byrne, B. M. (1998). Structural Equation Modeling with LISREL, PRELIS, and SIMPLIS: Basic concepts, applications, and programming. New York, NY: Psychology Press.

Byrne, B. M., Shavelson, R. J., \& Muthén, B. O. (1989). Testing for the equivalence of factor covariance and mean structures: The issue of partial measurement invariance. Psychological Bulletin, 105(3), 456-466. https://doi.org/10.1037/0033-2909.105.3.456

Cambré, B., Welkenhuysen-Gybels, J., \& Billiet, J. (2002). Is it content or style? An evaluation of two competitive measurement models applied to a balanced set of ethnocentrism items. International Journal of Comparative Sociology, 43, 1-20.

https://doi.org/10.1177/002071520204300101.

Cattell, R. B. (1986). The psychometric properties of tests: Consistency, validity, and efficiency. In R. B. Cattell \& R. C. Johnson (Eds.), Functional Psychological Testing (pp. 54-78). New York, NY: Brunner/Mazel.

Chen, F. F. (2007). Sensitivity of goodness of fit indexes to lack of measurement invariance. Structural Equation Modeling, 14(3), 464-504. https://doi.org/10.1080/10705510701301834

Chen, F. F. (2008). What happens if we compare chopsticks with forks? The impact of making inappropriate comparisons in cross-cultural research. Journal of Personality and Social Psychology, 95(5), 1005-1018. https://doi.org/10.1037/a0013193

Cheung, G. W., \& Rensvold, R. B. (2002). Evaluating goodness-of-fit indexes for testing measurement invariance. Structural Equation Modeling, 9(2), 233-255. https://doi.org/10.1207/s15328007sem0902_5

Cho, E., \& Kim, S. (2015). Cronbach's coefficient alpha: Well known but poorly understood. Organizational Research Methods, 18(2), 207-230. https://doi.org/10.1177/1094428114555994

Church, A. T., \& Lonner, W. J. (1998). The cross-cultural perspective in the study of personality: Rationale and current research. Journal of Cross-Cultural Psychology, 29(1), 32-62. https://doi.org/10.1177/0022022198291003 
Clark, L., \& Watson, D. (2019). Constructing validity: New developments in creating objective measuring instruments. Psychological Assessment, 31(12), 1412-1427. https://doi.org/10.1037/pas0000626

Comrey, A. L., \& Lee, H. B. (2013). A first course in factor analysis $\left(2^{\text {nd }}\right.$ ed.) [eBook]. New York, NY: Psychology Press.

Cortina, J. M. (1993). What is coefficient alpha? An examination of theory and applications. Journal of Applied Psychology, 78(1), 98-104. https://doi.org/10.1037/0021-9010.78.1.98

Costa, P. T., \& McCrae, R. R. (1992). Revised NEO Personality Inventory (NEO-PI-R) and NEO Five-Factor Inventory (NEO-FFI) Professional Manual. Odessa, FL: Psychological Assessment Resources.

Costa, P. T., Jr., \& McCrae, R. R. (2008). The Revised NEO Personality Inventory (NEO-PI-R). In G. J. Boyle, G. Matthews, \& D. H. Saklofske (Eds.), The SAGE Handbook of Personality Theory and Assessment (Vol. 2): Personality Measurement and Testing (pp. 179-198). Thousand Oaks, CA: Sage. https://doi.org/10.4135/9781849200479.n9

Cronbach, L. J., \& Shavelson, R. J. (2004). My current thoughts on coefficient alpha and successor procedures. Educational and Psychological Measurement, 64(3), 391-418. https://doi.org/10.1177/0013164404266386

Cronbach, L. J., \& Meehl, P. (1955). Construct validity in psychological tests. Psychological Bulletin, 52(4), 281-302. https://doi.org/10.1037/h0040957

Crocker, L. M., \& Algina, J. (1986). Introduction to classical and modern test theory. New York, NY: Holt, Rinehart and Winston.

Dahlsgaard, K., Peterson, C., \& Seligman, M. E. P. (2005). Shared virtue: The convergence of valued human strengths across culture and history. Review of General Psychology, 9, 203-213. https://doi.org/10.1037/1089-2680.9.3.203

Danner, D., Blasius, J., Breyer, B., Eifler, S., Menold, N., Paulhus, D., Rammstedt, D., Roberts, R., Schmitt, M., \& Ziegler, M. (2016). Current challenges, new developments, and future directions in scale construction. European Journal of Psychological Assessment, 32(3), 175-180. https://doi.org/10.1027/1015-5759/a000375 
Danner, D., Rammstedt, B., Bluemke, M., Lechner, C., Berres, S., Knopf, T., Soto, C., \& John, O. P. (2019). Das Big-Five Inventar 2: Validierung eines Persönlichkeitsinventars zur Erfassung von 5 Persönlichkeitsdomänen und 15 Facetten [The German Big Five Inventory 2: Measuring five personality domains and 15 facets]. Diagnostica, 65(3), 121-132. https://doi.org/10.1026/0012$1924 / \mathrm{a} 000218$

Davis, L. (1992). Instrument review: Getting the most from a panel of experts. Applied Nursing Research, 5(4), 194-197. https://doi.org/10.1016/s0897-1897(05)80008-4

De Beuckelaer, A., Weijters, B., \& Rutten, A. (2010). Using ad hoc measures for response styles: A cautionary note. Quality and Quantity, 44(4), 761-775. https://doi.org/10.1007/s11135-0099225-z

Denissen, J. J. A., Luhmann, M., Chung, J. M., \& Bleidorn, W. (2019). Transactions between life events and personality traits across the adult lifespan. Journal of Personality and Social Psychology, 116(4), 612-633. https://doi.org/10.1037/pspp0000196

DeYoung, C. G., Quilty, L. C., \& Peterson, J. B. (2007). Between facets and domains: 10 aspects of the Big Five. Journal of Personality and Social Psychology, 93(5), 880-896. https://doi.org/10.1037/0022-3514.93.5.880

Diamond, M. R., O'Brien-Malone, A., \& Woodworth, R. J. (2010). Scoring the VIA Survey of Character. Psychological Reports, 107, 833--836.

Diener, E. D., Emmons, R. A., Larsen, R. J., \& Griffin, S. (1985). The Satisfaction with Life Scale. Journal of Personality Assessment, 49(1), 71-75. https://doi.org/10.1207/s15327752jpa4901_13

Dunn, T. J., Baguley, T., \& Brunsden, V. (2014). From Alpha to Omega: A practical solution to the pervasive problem of internal consistency estimation. British Journal of Psychology, 105, 399-412. https://doi.org/10.1111/bjop.12046

du Plessis, G. A., \& de Bruin, G. P. (2015). Using Rasch modelling to examine the international personality item pool (IPIP) values in action (VIA) measure of character strengths. Journal of Psychology in Africa, 25(6), 512-521. https://doi.org/10.1080/14330237.2015.1124603 
Dziuban, C. D., \& Shirkey, E. C. (1974). When is a correlation matrix appropriate for factor analysis? Some decision rules. Psychological Bulletin, 81(6), 358-361. https://doi.org/10.1037/h0036316

Eisinga, R., te Grotenhuis, M., \& Pelzer, B. (2013). The reliability of a two-item scale: Pearson, Cronbach, or Spearman-Brown? International Journal of Public Health, 58(4), 637-642. https://doi.org/10.1007/s00038-012-0416-3

Fabrigar, L. R., Wegener, D. T., MacCallum, R. C., \& Strahan, E. J. (1999). Evaluating the use of exploratory factor analysis in psychological research. Psychological Methods, 4, 272-299. https://doi.org/10.1037/1082-989X.4.3.272

Fan, X., \& Sivo, S. A. (2009). Using $\Delta$ Goodness-of-Fit indexes in assessing mean structure invariance. Structural Equation Modeling, 16(1), 54-69. https://doi.org/10.1080/10705510802561311

Feingold, A. (1994). Gender differences in personality: A meta-analysis. Psychological Bulletin, 116(3), 429-456. https://doi.org/10.1037/0033-2909.116.3.429

Feraco, T., Casali, N., \& Meneghetti, C. (2021). Do strengths converge into virtues? An item-, virtue-, and scale-level analysis of the Italian Values in Action Inventory of Strengths-120. Journal of Personality Assessment. https://doi.org/10.1080/00223891.2021.1934481

Ferrando, P. J., \& Lorenzo-Seva, U. (2018). Assessing the quality and appropriateness of factor solutions and factor score estimates in exploratory item factor analysis. Educational and Psychological Measurement, 78, 762-780. https://doi.org/10.1177/0013164417719308

Fischer, R., \& Poortinga, Y. H. (2018). Addressing methodological challenges in culture-comparative research. Journal of Cross-Cultural Psychology, 49, 691-712.

https://doi.org/10.1177/0022022117738086

Fleiss, J. L. (1971). Measuring nominal scale agreement among many raters. Psychological Bulletin, 76(5), 378-382. https://doi.org/10.1037/h0031619

Fornell, C., \& Larcker, D. F. (1981). Evaluating structural equation models with unobservable variables and measurement error. Journal of Marketing Research, 18(1), 39-50. https://doi.org/10.2307/3151312 
Fowler, F. J., Jr, \& Cosenza, C. (2008). Writing effective questions. In E. D. de Leeuw, J. J. Hox, \& D. A. Dillman (Eds), International Handbook of Survey Methodology (pp. 136-160). London, UK: Psychological Press.

Freelon, D. (2010). ReCal: Intercoder reliability calculation as a web service. International Journal of Internet Science, 5(1), 20-33.

Galton, F. (1884/1971). The measurement of character. In L. D. Goodstein \& R. I. Lanyon (Eds.), Readings in personality assessment (pp. 4-10). New York, NY: Wiley. (Reprinted from Fortnightly Review (of London), 1884, 42, 179-185)

Gander, F., Hofmann, J., \& Ruch, W. (2020). Character strengths: Person-environment fit and relationships with job and life satisfaction. Frontiers in Psychology, 11, 1582.

https://doi.org/10.3389/fpsyg.2020.01582

Garrido, L. E., Abad, F. J., \& Ponsoda, V. (2011). Performance of Velicer's minimum average partial factor retention method with categorical variables. Educational and Psychological Measurement, 71(3), 551-570. https://doi.org/10.1177/0013164410389489

Gauckler, B., \& Körner, T. (2011). Measuring the employment status in the labour force survey and the German Census 2011: Insights from recent research at Destatis. Methoden, Daten, Analysen (mda), 5, 181-205. https://nbn-resolving.org/urn:nbn:de:0168-ssoar-28213

Gennerich, C., \& Huber, S. (2006). Value priorities and content of religiosity - new research perspectives. Archiv für Religionspsychologie/Archive for the Psychology of Religion, 28(1), 253-267. https://doi.org/10.1163/008467206777832508

Gerbing, D. W., \& Anderson, J. C. (1988). An updated paradigm for scale development incorporating unidimensionality and its assessment. Journal of Marketing Research, 25(2), 186-192. https://doi.org/10.2307/3172650

Gignac, G. E., \& Szodorai, E. T. (2016). Effect size guidelines for individual differences researchers. Personality and Individual Differences, 102, 74-78. https://doi.org/10.1016/j.paid.2016.06.069 
Goldberg, L. R. (1990). An alternative "description of personality": The Big-Five factor structure. Journal of Personality and Social Psychology, 59(6), 1216-1229. https://doi.org/10.1037/00223514.59.6.1216

Goldberg, L. R. (1999). A broad-bandwidth, public domain, personality inventory measuring the lower-level facets of several five-factor models. In I. Mervielde, I. Deary, F. De Fruyt \& F. Ostendorf (Eds.), Personality Psychology in Europe (Vol. 7, pp. 7-28). Tilburg, Netherlands: Tilburg University Press.

Goldberg, L. R. (2006). Doing it all bass-ackwards: The development of hierarchical factor structures from the top down. Journal of Research in Personality, 40(4), 347-358.

https://doi.org/10.1016/j.jrp.2006.01.001

Goldberg, L. R., Johnson, J. A., Eber, H.W., Hogan, R., Ashton, M. C., Cloninger, C. R., \& Gough, H. G. (2006). The international personality item pool and the future of public-domain personality measures. Journal of Research in Personality, 40, 84-96.

https://doi.org/10.1016/j.jrp.2005.08.007

Goldberg, L. R., \& Saucier, G. (2016). The Eugene-Springfield Community Sample: Information Available from the Research Participants (ORI Technical Report, Vol. 56, No. 1). Eugene, OR: Oregon Research Institute.

Gorsuch, R. L. (1983/2015). Factor Analysis [2 ${ }^{\text {nd }}$ ed./Classic Edition]. New York, NY: Routledge/Taylor \& Francis.

Graham, J. M. (2006). Congeneric and (essentially) tau-equivalent estimates of score reliability: What they are and how to use them. Educational and Psychological Measurement, 66(6), 930-944. https://doi.org/10.1177/0013164406288165

Green, B. F., Bock, R. D., Humphreys, L. G., Linn, R. L., \& Reckase, M. D. (1984). Technical guidelines for assessing computerized adaptive tests. Journal of Educational Measurement, 21(4), 347-360. https://doi.org/10.1111/j.1745-3984.1984.tb01039.x 
Green, S., Lissitz, R., \& Mulaik, S. (1977). Limitations of coefficient Alpha as an index of test unidimensionality. Educational and Psychological Measurement, 37, 827-838. https://doi.org/10.1177/001316447703700403

Greenleaf, E. A. (1992). Improving rating scale measures by detecting and correcting bias components in some response styles. Journal of Marketing Research, 29, 176-188. https://doi.org/10.1177/002224379202900203

Grice, J. W. (2001). Computing and evaluating factor scores. Psychological Methods, 6, 430-450. https://doi.org/10.1037/1082-989X.6.4.430

Guion, R. (1977). Content validity: The source of my discontent. Applied Psychological Measurement, 1, 1-10. https://doi.org/10.1177/014662167700100103

Guttman, L. (1945). A basis for analyzing test-retest reliability. Psychometrika, 10(4), 255-282. https://doi.org/10.1007/bf02288892

Hair, J. F., Black, W. C., Babin, B. J., Anderson, R. E., \& Tatham, R. L. (2006). Multivariate data analysis (6th ed.). Upper Saddle River, N.J.: Pearson Prentice Hall.

Hallgren, K. A. (2012). Computing inter-rater reliability for observational data: An overview and tutorial. Tutorials in Quantitative Methods for Psychology, 8(1), 23-34. https://doi.org/10.20982/tqmp.08.1.p023

Hallquist, M. N., \& Wiley, J. F. (2018). MplusAutomation: An R package for facilitating large-scale latent variable analyses in Mplus. Structural Equation Modeling, 25(4), 621-638. https://doi.org/10.1080/10705511.2017.1402334

Hancock, G. R., \& Mueller, R. O. (2001). Rethinking construct reliability within latent variable systems. In R. Cudeck, S. du Toit, \& D. Sørbom (Eds.), Structural Equation Modeling: Present and FutureA Festschrift in Honor of Karl Jöreskog (pp. 195-216). Lincolnwood, IL: Scientific Software International.

Harkness, J. (2003). Questionnaire translation. In J. Harkness, F. J. R. van de Vijver \& P. Ph. Mohler (Eds.), Cross-Cultural Survey Methods (pp. 35-56). Hoboken, NJ: Wiley. 
Hattie, J. (1985). Methodology review: Assessing unidimensionality of tests and items. Applied Psychological Measurement, 9(2), 139-164. https://doi.org/10.1177/014662168500900204

Heene, M., Hilbert, S., Draxler, C., Ziegler, M., \& Bühner, M. (2011). Masking misfit in confirmatory factor analysis by increasing unique variances: A cautionary note on the usefulness of cutoff values of fit indices. Psychological Methods, 16(3), 319-336. https://doi.org/10.1037/a0024917

Heene, M., Bollmann, S., \& Bühner, M. (2014). Much ado about nothing, or much to do about something? Effects of scale shortening on criterion validity and mean differences. Journal of Individual Differences, 35, 245-249. https://doi.org/10.1027/1614-0001/a000146

Hofstee, W. K., De Raad, B., \& Goldberg, L. R. (1992). Integration of the Big Five and circumplex approaches to trait structure. Journal of Personality and Social Psychology 63(1), 146-163. https://doi.org/10.1037/0022-3514.63.1.146

Hopwood, C. J., \& Donnellan, M. B. (2010). How should the internal structure of personality inventories be evaluated? Personality and Social Psychology Review, 14(3), 332-346. https://doi.org/10.1177/1088868310361240

Horn, J. L. (1965). A rationale and test for the number of factors in factor analysis. Psychometrika, 30(2), 179-185. https://doi.org/10.1007/bf02289447

Hu, L., \& Bentler, P. M. (1999). Cutoff criteria for fit indexes in covariance structure analysis: Conventional criteria versus new alternatives. Structural Equation Modeling, 6(1), 1-55. https://doi.org/10.1080/10705519909540118

John, O. P., Angleitner, A., \& Ostendorf, F. (1988). The lexical approach to personality: A historical review of trait taxonomic research. European Journal of Personality, 2(3), 171-203. https://doi.org/10.1002/per.2410020302 John, O. P., \& Robins, R. W. (1993). Determinants of interjudge agreement on personality traits: The Big Five domains, observability, evaluativeness, and the unique perspective of the self. Journal of Personality, 61(4), 521-551. https://doi.org/10.1111/j.1467-6494.1993.tb00781.x 
John, O. P., \& Soto, C. J. (2007). The importance of being valid: Reliability and the process of construct validation. In R. W. Robins, R. C. Fraley, \& R. F. Krueger, (Eds), Handbook of research methods in personality psychology (pp. 461-494). New York, NY: Guilford.

Kaiser, H. F., \& Rice, J. (1974). Little Jiffy, Mark IV. Educational and Psychological Measurement, 34(1), 111-117. https://doi.org/10.1177/001316447403400115

Kam, C. C. S., \& Meyer, J. P. (2015). How careless responding and acquiescence response bias can influence construct dimensionality: The case of job satisfaction. Organizational Research Methods, 18(3), 512-541. https://doi.org/10.1177/1094428115571894

Kandler, C., Zimmermann, J., \& McAdams, D. P. (2014). Core and surface characteristics for the description and theory of personality differences and development. European Journal of Personality, 28, 231-243. https://doi.org/10.1002/per.1952

Kemper, C. J., Trapp, S., Kathmann, N., Samuel, D. B., \& Ziegler, M. (2019). Short versus long scales in clinical assessment: Exploring the trade-off between resources saved and psychometric quality lost using two measures of obsessive-compulsive symptoms. Assessment, 26(5), 767-782. https://doi.org/10.1177/1073191118810057

Kenny, D. A., Kaniskan, B., \& McCoach, D. B. (2015). The performance of RMSEA in models with small degrees of freedom. Sociological Methods and Research, 44(3), 486-507. https://doi.org/10.1177/0049124114543236

Kenny, D. A., \& McCoach, D. B. (2003). Effect of the number of variables on measures of fit in structural equation modeling. Structural Equation Modeling, 10(3), 333-351. https://doi.org/10.1207/S15328007SEM1003_1

Kim, K. H. (2005). The relation among fit indexes, power, and sample size in structural equation modeling. Structural Equation Modeling, 12, 368-390. https://doi.org/10.1207/s15328007sem1203_2

Kline, R. B. (2011). Principles and practice of structural equation modeling (3rd ed.) New York, NY: Guilford. 
Koo, T. K., \& Li, M. Y. (2016). A guideline of selecting and reporting intraclass correlation coefficients for reliability research. Journal of Chiropractic Medicine, 15(2), 155-163. https://doi.org/10.1016/j.jcm.2016.02.012

Landis, J. R., \& Koch, G. G. (1977). The measurement of observer agreement for categorical data. Biometrics, 33(1), 159-174. https://doi.org/10.2307/2529310

Lavy, S., \& Benish-Weisman, M. (2021). Character strengths as "Values in Action": Linking character strengths with values theory - an exploratory study of the case of gratitude and self-transcendence. Frontiers in Psychology, 12, 576189. https://doi.org/10.3389/fpsyg.2021.576189

Lawshe, C. H. (1975). A quantitative approach to content validity. Personnel Psychology, 28, 563-575. https://doi.org/10.1111/j.1744-6570.1975.tb01393.x

Lechner, C. M., Bhaktha, N., Groskurth, K., \& Bluemke, M. (2021). Why ability point estimates can be pointless: A primer on using skill measures from large-scale assessments in secondary analyses. Measurement Instruments for the Social Sciences, 3, 2. https://doi.org/10.1186/s42409-02000020-5

Lechner, C. M., Partsch, M. V., Danner, D., \& Rammstedt, B. (2019). Individual, situational, and cultural correlates of acquiescent responding: Towards a unified conceptual framework. British Journal of Mathematical and Statistical Psychology, 72(3), 426-446. https://doi.org/10.1111/bmsp.12164

Lechner, C. M., \& Rammstedt, B. (2015). Cognitive ability, acquiescence, and the structure of personality in a sample of older adults. Psychological Assessment, 27(4), 1301-1311. https://doi.org/10.1037/pas0000151

Leiner, D. J. (2019). Too fast, too straight, too weird: Non-reactive indicators for meaningless data in internet surveys. Survey Research Methods, 13(3), 229-248. https://doi.org/10.18148/srm/2018.v13i3.7403

Lim, S., \& Jahng, S. (2019). Determining the number of factors using parallel analysis and its recent variants. Psychological Methods, 24(4), 452-467. https://doi.org/10.1037/met0000230 
Liu, D. (2012). Translation and culture: Translating idioms between English and Chinese from a cultural perspective. Theory and Practice in Language Studies, 2(11), 2357-2362. https://doi.org/10.4304/tpls.2.11.2357-2362

Lorenzo-Seva, U. (2013). How to report the percentage of explained common variance in exploratory factor analysis. Technical Report. Department of Psychology, Universitat Rovira i Virgili, Tarragona. http://psico.fcep.urv.es/utilitats/factor/documentation/Percentage_of_explained_common_variance.pdf

Lorenzo-Seva, U., \& Ferrando, P. J. (2006). FACTOR: A computer program to fit the exploratory factor analysis model. Behavioral Research Methods, 38, 88-91. https://doi.org/10.3758/BF03192753 Lorenzo-Seva, U., \& Ferrando, P. J. (2013). FACTOR 9.2 - A comprehensive program for fitting exploratory and semiconfirmatory factor analysis and IRT models. Applied Psychological Measurement, 37(6), 497-498. https://doi.org/10.1177/0146621613487794

MacDonald, D. A., Friedman, H. L., Brewczynski, J., Holland, D., Salagame, K.K.K., Mohan, K.K., Gubrij, Z. O., \& Cheong, H. W. (2015). Spirituality as a scientific construct: Testing its universality across cultures and languages. PLoS One, 10(3), e0117701. https://doi.org/ 10.1371/journal.pone.0117701

Marsh, H. W., \& Grayson, D. (1995). Latent variable models of multitrait-multimethod data. In R. H. Hoyle (Ed.), Structural equation modeling: Concepts, issues, and applications (pp. 177-198). Thousand Oaks, CA: Sage.

Marsh, H. W., Hau, K., \& Wen, Z. (2004). In search of golden rules: Comment on hypothesis-testing approaches to setting cutoff values for fit indexes and dangers in overgeneralizing Hu and Bentler's (1999) findings. Structural Equation Modeling, 11(3), 320-341. https://doi.org/10.1207/s15328007sem1103_2

Martínez-Martí, M. L., \& Ruch, W. (2017). Character strengths predict resilience over and above positive affect, self-efficacy, optimism, social support, self-esteem, and life satisfaction. Journal of Positive Psychology, 12, 110-119. https://doi.org/10.1080/17439760.2016.1163403 
McCrae, R. R., Herbst, J. H., \& Costa, P. T., Jr. (2001). Effects of acquiescence on personality factor structures. In R. Riemann, F. Ostendorf, \& F. Spinath (Eds.), Personality and temperament: Genetics, evolution, and structure (pp. 217-231). Berlin, Germany: Pabst Science.

McCrae, R. R., \& John, O. P. (1992). An introduction to the five-factor model and its applications. Journal of Personality, 60, 175-215. https://doi.org/10.1111/j.1467-6494.1992.tb00970.x

McCrae, R. R., Kurtz, J. E., Yamagata, S., \& Terracciano, A. (2011). Internal consistency, retest reliability, and their implications for personality scale validity. Personality and Social Psychology Review, 15(1), 28-50. https://doi.org/10.1177/1088868310366253

McDonald, R. P. (1999). Test theory: A unified treatment. Hillsdale, NJ: Lawrence Erlbaum Associates Publishers.

McGrath, R. E. (2014). Scale- and item-level factor analyses of the VIA Inventory of Strengths. Assessment, 21(1), 4-14. https://doi.org/10.1177/1073191112450612

McGrath, R. E. (2015). Integrating psychological and cultural perspectives on virtue: The hierarchical structure of character strengths. The Journal of Positive Psychology, 10(5), 407-424. https://doi.org/10.1080/17439760.2014.994222

McGrath, R. E. (2016). Measurement invariance in translations of the VIA inventory of strengths. European Journal of Psychological Assessment, 32(3), 187-194. https://doi.org/10.1027/1015$5759 / a 000248$

McGrath, R. E. (2017). The VIA assessment suite for adults: Development and evaluation [Technical report]. Cincinnati, OH: VIA Institute on Character.

McGrath, R. E. (2019). Technical report: The VIA Assessment Suite for Adults: Development and initial evaluation (rev ed.). VIA Institute on Character.

McGrath, R. E., Hall-Simmonds, A., \& Goldberg, L. R. (2020). Are measures of character and personality distinct? Evidence from observed-score and true-score analyses. Assessment, 27(1), 117-135. https://doi.org/10.1177/1073191117738047 
McGrath, R. E., \& Wallace, N. (2021). Cross-validation of the VIA Inventory of Strengths-Revised and its short forms. Journal of Personality Assessment, 103(1), 120-131. https://doi.org/10.1080/00223891.2019.1705465

McNeish, D. (2018). Thanks coefficient alpha, we'll take it from here. Psychological Methods, 23(3), 412-433. https://doi.org/10.1037/met0000144

Meade, A. W. \& Craig, S. B. (2012). Identifying careless responses in survey data. Psychological Methods, 17(3), 437-455. https://doi.org/10.1037/a0028085

Meredith, W. (1993). Measurement invariance, factor analysis and factorial invariance. Psychometrika, 58(4), 525-543. https://doi.org/10.1007/bf02294825

Meredith, W., \& Millsap, R. E. (1992). On the misuse of manifest variables in the detection of measurement bias. Psychometrika, 57(2), 289-311. https://doi.org/10.1007/bf02294510

Morizot, J., Ainsworth, A. T., \& Reise, S. P. (2007). Toward modern psychometrics: Application of item response theory models in personality research. In R. W. Robins, R. C. Fraley, \& R. F. Krueger (Eds.), Handbook of research methods in personality (pp. 407-423). New York, NY: Guilford.

Mõttus, R., Wood, D., Condon, D. M., Back, M. D., Baumert, A., Costantini, G., Epskamp, S., Greiff, S., Johnson, W., Lukaszewski, A., Murray, A., Revelle, W., Wright, A. G. C., Yarkoni, T., Ziegler, M., and Zimmermann, J. (2020). Descriptive, predictive and explanatory personality research: Different goals, different approaches, but a shared need to move beyond the Big Few traits. European Journal of Personality, 34(6), 1175-1201. https://doi.org/10.1002/per.2311

Muthén, B. O., du Toit, S.H.C., \& Spisic, D. (1997). Robust inference using weighted least squares and quadratic estimating equations in latent variable modeling with categorical and continuous outcomes. Unpublished technical report. College of Education, UCLA, Los Angeles, CA. https://www. statmodel.com/download/Article_075.pdf

Muthén, L. K., \& Muthén, B. O. (1998-2012). Mplus User's Guide (version 7, rev.3). Los Angeles, CA: Muthén \& Muthén. 
Nießen, D., Groskurth, K., Rammstedt, B., \& Lechner, C. M. (2020). An English-language adaptation of the General Life Satisfaction Short Scale (L-1). Zusammenstellung sozialwissenschaftlicher Items und Skalen (ZIS). https://doi.org/10.6102/zis284

Ng, V., Cao, M., Marsh, H. W., Tay, L., \& Seligman, M. E. P. (2017). The factor structure of the Values in Action Inventory of Strengths (VIA-IS): An item-level exploratory structural equation modeling (ESEM) bifactor analysis. Psychological Assessment, 29(8), 1053-1058.

https://doi.org/10.1037/pas0000396

Noftle, E. E., Schnitker, S. A., \& Robins, R. W. (2011). Character and personality: Connections between positive psychology and personality psychology. In K. M. Sheldon, T. B. Kashdan, \& M. F. Steger (Eds.), Designing positive psychology: Taking stock and moving forward (pp. 207-227). Oxford, UK: Oxford University Press.

Nunnally, J. C., \& Bernstein, I. H. (1994). Psychometric theory (3rd ed.). New York, NY: McGraw-Hill.

Ones, D. S., \& Viswesvaran, C. (1996). Bandwidth-fidelity dilemma in personality measurement for personnel selection. Journal of Organizational Behavior, 17(6), 609-626. https://doi.org/10.1002/(SICI)1099-1379(199611)17:6<609::AID-JOB1828>3.0.CO;2-K

Park, N. (2018). Classifying and measuring strengths of character. In C. R. Snyder, S. J. Lopez, L. M. Edwards, \& S. C. Marques (Eds.), Oxford Handbook of Positive Psychology (3rd ed.). Oxford University Press. https://doi.org/10.1093/oxfordhb/9780199396511.001.0001

Park, N., Barton, M., \& Pillay, J. (2017). Strengths of character and virtues: What we know and what we still want to learn. In M. Warren and D. Stewart (Eds.), Scientific Advances in Positive Psychology (pp. 73-101). Santa Barbara, CA: Praeger Publishers.

Park, N., \& Peterson, C. (2010). Does it matter where we live? The urban psychology of character strengths. American Psychologist, 65(6), 535-547. https://doi.org/10.1037/a0019621

Park, N., Peterson, C., \& Seligman, M. E. P. (2004). Strengths of Character and Well-Being. Journal of Social and Clinical Psychology, 23(5), 603-619. https://doi.org/10.1521/jscp.23.5.603.50748 
Park, N., Peterson, C., \& Seligman, M. E. P. (2006). Character strengths in fifty-four nations and the fifty US states. Journal of Positive Psychology, 1(3), 118-129. https://doi.org/10.1080/17439760600619567

Partsch, M. V., Bluemke, M., \& Lechner, C. M. (2021). Revisiting the hierarchical structure of the 24 VIA character strengths: Three global dimensions may suffice to capture their essence. European Journal of Personality. https://doi.org/10.1177/08902070211017760

Peterson, C. (2003). The Values-in-Action Structured Interview (VIA-SI). Cincinnati: The VIA Institute on Character.

Peterson, C., \& Park, N. (2009). Classifying and measuring strengths of character. In S. J. Lopez \& C. R. Snyder (Eds.), Oxford handbook of positive psychology (2nd ed., pp. 25-33). New York, NY: Oxford University Press.

Peterson, C., Park, N., \& Seligman, M. E. P. (2005). Assessment of character strengths. In G. P. Koocher, J. C. Norcross, \& S. S. Hill III. (Eds.), Psychologists' desk reference (2 ${ }^{\text {nd }}$ ed., pp. 93-98). New York, NY: Oxford University Press.

Peterson, C., \& Seligman, M. E. P. (2003). Character strengths before and after September 11. Psychological Science, 14(4), 381-384. https://doi.org/10.1111/1467-9280.24482

Peterson, C., \& Seligman, M. E. P. (2004). Character strengths and virtues: A handbook and classification. New York, NY: Oxford University Press and Washington, DC: American Psychological Association.

Piedmont, R. L. (2007). Cross-cultural generalizability of the Spiritual Transcendence Scale to the Philippines: Spirituality as a human universal. Mental Health, Religion and Culture, 10(2), 89-107. https://doi.org/10.1080/13694670500275494

Podsakoff, P. M., MacKenzie, S. B., \& Podsakoff, N. P. (2012). Sources of method bias in social science research and recommendations on how to control it. Annual Review of Psychology, 63(1), 539569. https://doi.org/10.1146/annurev-psych-120710-100452 
Polit, D. F., Beck, C. T., \& Owen, S. V. (2007). Is the CVI an acceptable indicator of content validity? Appraisal and recommendations. Research in Nursing and Health, 30, 459-467. https://doi.org/10.1002/nur.20199

Primi, R., De Fruyt, F., Santos, D., Antonoplis, S., \& John, O. P. (2020). True or false? Keying direction and acquiescence influence the validity of socio-emotional skills items in predicting high school achievement. International Journal of Testing, 20(2), 97-121. https://doi.org/10.1080/15305058.2019.1673398

Proyer, R. T., Gander, F., Wellenzohn, S., \& Ruch, W. (2013). What good are character strengths beyond subjective well-being? The contribution of the good character on self-reported health-oriented behavior, physical fitness, and the subjective health status. The Journal of Positive Psychology, 8(3), 222-232. https://doi.org/10.1080/17439760.2013.777767

Proyer, R. T., Gander, F., Wyss, T., \& Ruch, W. (2011). The Relation of Character Strengths to Past, Present, and Future Life Satisfaction among German-Speaking Women. Applied Psychology: Health and Well-Being, 3(3), 370-384. https://doi.org/10.1111/j.1758-0854.2011.01060.x

Putnick, D. L., \& Bornstein, M. H. (2016). Measurement invariance conventions and reporting: The state of the art and future directions for psychological research. Developmental Review, 41, 7190. https://doi.org/10.1016/j.dr.2016.06.004

Rammstedt, B., \& Beierlein, C. (2014). Can't we make it any shorter? The limits of personality assessment and ways to overcome them. Journal of Individual Differences, 35(4), 212-220. https://doi.org/10.1027/1614-0001/a000141

Rammstedt, B., \& Farmer, R. F. (2013). The impact of acquiescence on the evaluation of personality structure. Psychological Assessment, 25(4), 1137-1145. https://doi.org/10.1037/a0033323

Rammstedt, B., Lechner, C. M., \& Danner, D. (2020). Short forms do not fall short: A comparison of three (extra-)short forms of the Big Five [Advance online publication]. European Journal of Psychological Assessment. https://doi.org/10.1027/1015-5759/a000574

Raykov, T. (1997). Estimation of composite reliability for congeneric measures. Applied Psychological Measurement, 21(2), 173-184. https://doi.org/10.1177/01466216970212006 
Raykov, T. (1998). Coefficient alpha and composite reliability with interrelated nonhomogeneous items. Applied Psychological Measurement, 22(4), 375-385. https://doi.org/10.1177/014662169802200407

Raykov, T. (2001). Bias of coefficient alpha for fixed congeneric measures with correlated errors. Applied Psychological Measurement, 25(1), 69-76. https://doi.org/10.1177/01466216010251005

R Core Team (2018). R: A language and environment for statistical computing. R Foundation for Statistical Computing, Vienna, Austria. http://www.R-project.org/

Revelle, W. (2019). psych: Procedures for Psychological, Psychometric, and Personality Research [R package version 1.8.12]. Northwestern University, Evanston, Illinois. http://CRAN.R-project.org/package=psych

Rhemtulla, M., Brosseau-Liard, P. É., \& Savalei, V. (2012). When can categorical variables be treated as continuous? A comparison of robust continuous and categorical SEM estimation methods under suboptimal conditions. Psychological Methods, 17(3), 354-373. https://doi.org/10.1037/a0029315

Rodriguez, A., Reise, S. P., \& Haviland, M. G. (2016). Applying bifactor statistical indices in the evaluation of psychological measures. Journal of Personality Assessment, 98, 223-237. https://doi.org/10.1080/00223891.2015.1089249

Rose, N., Wagner, W., Mayer, A., \& Nagengast, B. (2019). Model-based manifest and latent composite scores in structural equation models. Collabra: Psychology, 5(1), 9. https://doi.org/10.1525/collabra.143

Rosenberg, S., Nelson, C., \& Vivekananthan, P. S. (1968). A multidimensional approach to the structure of personality impressions. Journal of Personality and Social Psychology, 9(4), 283-294. https://doi.org/10.1037/h0026086

Rosseel, Y. (2012). lavaan: An R package for structural equation modeling. Journal of Statistical Software, 48(2), 1-36. http://www.jstatsoft.org/v48/i02/

Ruch, W., Huber, A., Beermann, U., \& Proyer, R.T. (2007). Character strengths as predictors of the "good life" in Austria, Germany and Switzerland. In: Romanian Academy - "George Barit" 
Department of Social Research (Ed.), Studies and Researches in Social Sciences (pp. 123-131). Academia Română. https://doi.org/ 10.5167/uzh-3648

Ruch, W., Martínez-Martí, M. L., Proyer, R. T., \& Harzer, C. (2014). The Character Strengths Rating Form (CSRF): Development and initial assessment of a 24-Item rating scale to assess character strengths. Personality and Individual Differences, 68, 53-58.

https://doi.org/10.1016/j.paid.2014.03.042

Ruch, W., \& Proyer, R. T. (2015). Mapping strengths into virtues: the relation of the 24 VIA-strengths to six ubiquitous virtues. Frontiers in Psychology, 6, 460.

https://doi.org/10.3389/fpsyg.2015.00460

Rutkowski, L., \& Svetina, D. (2014). Assessing the hypothesis of measurement invariance in the context of large-scale international surveys. Educational and Psychological Measurement, 74(1), 3157.

Satorra, A. \& Bentler, P.M. (2010). Ensuring positiveness of the scaled difference chi-square test statistic. Psychometrika, 75, 243-248. https://doi.org/10.1007/s11336-009-9135-y

Saroglou, V. (2011). Believing, Bonding, Behaving, and Belonging: The big four religious dimensions and cultural variation. Journal of Cross-Cultural Psychology, 42(8), 1320-1340. https://doi.org/10.1177/0022022111412267

Saroglou, V., Delpierre, V., \& Dernelle, R. (2004). Values and religiosity: A meta-analysis of studies using Schwartz's model. Personality and Individual Differences, 37(4), 721-734. https://doi.org/10.1016/j.paid.2003.10.005

Saucier, G. (2009). Recurrent personality dimensions in inclusive lexical studies: Indications for a Big Six Structure. Journal of Personality, 77(5), 1577-1614. https://doi.org/10.1111/j.14676494.2009.00593.x

Saucier, G., \& Goldberg, L. R. (1996). The language of personality: Lexical perspectives on the five-factor model. In J. S. Wiggins (Ed.), The Five-Factor Model of Personality (pp. 21-50). New York, NY: Guilford. 
Saucier, G., \& Goldberg, L. R. (1998). What is beyond the Big Five? Journal of Personality, 66(4), 495524. https://doi.org/10.1111/1467-6494.00022

Saucier, G., \& lurino, K. (2020). High-dimensionality personality structure in the natural language: Further analysis of classic sets of English-language trait-adjectives. Journal of Personality and Social Psychology, 119, 1188-1219. https://doi.org/10.1037/pspp0000273

Saucier, G., \& Skrzypińska, K. (2006). Spiritual but not religious? Evidence for two independent dimensions. Journal of Personality, 74(5), 1257-1292. https://doi.org/10.1111/j.14676494.2006.00409.x

Schimmack, U., Böckenholt, U., \& Reisenzein, R. (2002). Response styles in affect ratings: Making a mountain out of a molehill. Journal of Personality Assessment, 78, 461-483.

Schmitt, N. (1996). Uses and abuses of coefficient alpha. Psychological Assessment, 8(4), 350-353. https://doi.org/10.1037/1040-3590.8.4.350

Schroeders, U., Wilhelm, O., \& Olaru, G. (2016). Meta-heuristics in short scale construction: Ant colony optimization and genetic algorithm. PLoS One, 11(11), e0167110. https://doi.org/10.1371/journal.pone.0167110

Schumacker, R. E., \& Lomax, R. G. (2004). A beginner's guide to structural equation modeling (2nd ed.). Mahwah, NJ: Lawrence Erlbaum Associates.

Schwartz, S. H. (1992). Universals in the content and structure of values: Theoretical advances and empirical tests in 20 countries. In M. P. Zanna (Ed.), Advances in Experimental Social Psychology (Vol. 25, pp. 1-65). San Diego, CA, US: Academic Press. https://doi.org/10.1016/S00652601(08)60281-6

Schwartz, S. H. (1994). Are there universal aspects in the structure and contents of human values? Journal of Social Issues, 50(4), 19-45. https://doi.org/10.1111/j.1540-4560.1994.tb01196.x Schwartz, S. H. (2003a). A proposal for measuring value orientations across nations. In: Questionnaire Development Report of the European Social Survey (Chapter 7). Retrieved Oct 8, 2019, from the European Social Survey Data website https://www.europeansocialsurvey.org/docs/methodology/core_ess_questionnaire/ESS_core_questionnaire_human_values.pdf 
Schwartz, S. H. (2003b). Computing scores for the 10 human values (European Social Survey). Retrieved Oct 8, 2019 from the European Social Survey Data website https://www.europeansocialsurvey.org/docs/methodology/ESS_computing_human_values_scale.pdf

Schwartz, S. H. (2006). A theory of cultural value orientations: Explication and applications. Comparative Sociology, 5, 137-182. https://doi.org/10.1163/156913306778667357

Schwartz, S. H. (2013). Human Values. Retrieved Oct 8, 2019, from the European Social Survey Data website https://essedunet.nsd.uib.no/cms/topics/1

Schwartz, S. H., \& Bilsky, W. (1987). Toward a universal psychological structure of human values. Journal of Personality and Social Psychology, 53(3), 550-562. https://doi.org/10.1037/00223514.53.3.550

Schwartz, S. H., \& Bilsky, W. (1990). Toward a theory of the universal content and structure of values: Extensions and cross-cultural replications. Journal of Personality and Social Psychology, 58(8), 878-891. https://doi.org/10.1037/0022-3514.58.5.878

Schwartz, S. H., \& Boehnke, K. (2004). Evaluating the structure of human values with confirmatory factor analysis. Journal of Research in Personality, 38(3), 230-255. https://doi.org/10.1016/s0092-6566(03)00069-2

Schwartz, S. H., Breyer, B., \& Danner, D. (2015). Human Values Scale (ESS). Zusammenstellung sozialwissenschaftlicher Items und Skalen. https://doi.org/10.6102/zis234

Schwartz, S. H., Cieciuch, J., Vecchione, M., Davidov, E., Fischer, R., Beierlein, C., Ramos, A., Verkasalo, M., Lönnqvist, J. E., Demirutku, K., Dirilen-Gumus, O., \& Konty, M. (2012). Refining the theory of basic individual values. Journal of Personality and Social Psychology, 103(4), 663-688. https://doi.org/10.1037/a0029393

Schwartz, S. H., \& Huismans, S. (1995). Value priorities and religiosity in four Western religions. Social Psychology Quarterly, 58(2), 88-107. https://doi.org/10.2307/2787148

Schwartz, S. H., Melech, G., Lehmann, A., Burgess, S., \& Harris, M. (2001). Extending the cross-cultural validity of the theory of basic human values with a different method of measurement. 
Journal of Cross Cultural Psychology, 32(5), 519-542.

https://doi.org/10.1177/0022022101032005001

Schwartz, S. H., \& Sagiv, L. (1995). Identifying culture-specifics in the content and structure of values. Journal of Cross-Cultural Psychology, 26(1), 92-116.

https://doi.org/10.1177/0022022195261007

Schwarz, G. (1978). Estimating the dimension of a model. Annals of Statistics, 6(2), 461-464. https://doi.org/10.1214/aos/1176344136

Seligman, M.E. (2002). Authentic happiness: Using the new positive psychology to realize your potential for lasting fulfillment. New York, NY: Free Press/Simon and Schuster.

Seligman, M. E. P. (2011). Flourish: A visionary new understanding of happiness and well-being. New York, NY: Free Press/Simon and Schuster.

Seligman, M. E. P., Steen, T. A., Park, N., \& Peterson, C. (2005). Positive psychology progress: Empirical validation of interventions. American Psychologist, 60(5), 410-421.

https://doi.org/10.1037/0003-066X.60.5.410

Shapiro, A., \& Ten Berge, J. M. (2002). Statistical inference of minimum rank factor analysis. Psychometrika, 67(1), 79-94. https://doi.org/10.1007/BF02294710

Shi, D., Lee, T., \& Maydeu-Olivares, A. (2019). Understanding the model size effect on SEM fit indices. Educational and Psychological Measurement, 79(2), 310-334.

https://doi.org/10.1177/0013164418783530

Sijtsma, K. (2009). On the use, the misuse, and the very limited usefulness of Cronbach's alpha. Psychometrika, 74(1), 107-120. https://doi.org/10.1007/s11336-008-9101-0

Sireci, S. G. (1998). The construct of content validity. Social Indicators Research, 45(1/3), 83-117. https://doi.org/10.1023/A:1006985528729

Soto, C. J., \& John, O. P. (2017a). The next Big Five Inventory (BFI-2): Developing and assessing a hierarchical model with 15 facets to enhance bandwidth, fidelity, and predictive power. Journal of Personality and Social Psychology, 113(1), 117-143. https://doi.org/10.1037/pspp0000096 
Soto, C. J., \& John, O. P. (2017b). Short and extra-short forms of the Big Five Inventory-2: The BFI-2-S and BFI-2-XS. Journal of Research in Personality, 68, 69-81. https://doi.org/10.1016/j.jrp.2017.02.004

Soto, C.J., \& John, O. P. (2019). Optimizing the length, width, and balance of a personality scale: How do internal characteristics affect external validity? Psychological Assessment, 31(4), 444-459. https://doi.org/10.1037/pas0000586

Specht, J., Egloff, B., \& Schmukle, S. C. (2011). Stability and change of personality across the life course: The impact of age and major life events on mean-level and rank-order stability of the Big Five. Journal of Personality and Social Psychology, 101(4), 862-882. https://doi.org/10.1037/a0024950

Stahlmann, A. G., \& Ruch, W. (2020). Scrutinizing the criteria for character strengths: Laypersons assert that every strength is positively morally valued, even in the absence of tangible outcomes. Frontiers in Psychology, 11, 591028. https://doi.org/10.3389/fpsyg.2020.591028

Steiger, J. H. (1990). Structural model evaluation and modification: An interval estimation approach. Multivariate Behavioral Research, 25(2), 173-180. https://doi.org/10.1207/s15327906mbr2502_4

Steinmetz, H. (2013). Analyzing observed composite differences across groups: Is partial measurement invariance enough? Methodology: European Journal of Research Methods for the Behavioral and Social Sciences, 9(1), 1-12. https://doi.org/10.1027/1614-2241/a000049

Stout, W. (1987). A nonparametric approach for assessing latent trait unidimensionality. Psychometrika, 52(4), 589-617. https://doi.org/10.1007/bf02294821

Tabachnick, B. G., \& Fidell, L. S. (2013). Using Multivariate Statistics (6th ed.). Boston, MA Pearson. Ten Berge, J. M. F. (1998). Some recent developments in factor analysis and the search for proper communalities. In A. Rizzi, M. Vichi, \& H.-H. Bock (Eds.), Advances in data science and classification (pp. 325-334). Heidelberg, Germany: Springer.

Ten Berge, J. M. F., \& Kiers, H. A. L. (1991). A numerical approach to the approximate and the exact minimum rank of a covariance matrix. Psychometrika, 56(2), 309-315. 
Tilden, V., Nelson, C., \& May, B. (1990). Use of qualitative methods to enhance content validity. Nursing Research, 39(3), 172-175. https://doi.org/10.1097/00006199-199005000-00015

Timmerman, M. E., \& Lorenzo-Seva, U. (2011). Dimensionality assessment of order polytomous items with parallel analysis. Psychological Methods, 16(2), 209-220. https://doi.org/10.1037/a0023353

Thalmayer, A. G, \& Saucier, G. (2014). The questionnaire Big Six in 26 nations: Developing cross-culturally applicable Big Six, Big Five and Big Two Inventories. European Journal of Personality, 28(5), 482-496. https://doi.org/10.1002/per.1969

Thalmayer, A. G., Saucier, G., \& Eigenhuis, A. (2011). Comparative validity of brief to medium-length Big Five and Big Six Personality Questionnaires. Psychological Assessment, 23(4), 995-1009. https://doi.org/10.1037/a0024165

Valentini, F. (2017). Influência e controle da aquiescência na análise fatorial. Revista Avaliação Psicológica, 16(2), 116-119. https://doi.org/10.15689/ap.2017.1602.ed

Vandenberg, R. J., \& Lance, C. E. (2000). A review and synthesis of the measurement invariance literature: Suggestions, practices, and recommendations for organizational research. Organizational Research Methods, 3(1), 4-69. https://doi.org/10.1177/109442810031002

Vazire, S. (2010). Who knows what about a person? The Self-Other Knowledge Asymmetry (SOKA) model. Journal of Personality and Social Psychology, 98(2), 281-300. https://doi.org/10.1037/a0017908

Velicer, W. F., Eaton, C. A., \& Fava, J. L. (2000). Construct explication through factor or component analysis: A review and evaluation of alternative procedures for determining the number of factors or components. In R. D. Goffin \& E. Helmes (Eds.), Problems and solutions in human assessment: Honoring Douglas N. Jackson at Seventy (pp. 41-71). New York, NY: Kluwer Academic/Plenum.

VIA Institute on Character (2020, June 22). VIA assessments. https://www.viacharacter.org/researchers/assessments 
Vylobkova, V., Heintz, S., Gander, F., Wagner, L., \& Ruch, W. (2021, May 26). Convergence and psychometric properties of character strengths measures: The VIA-IS and the VIA-IS-R. PsyArxiv. https://doi.org/10.31234/osf.io/8zvjb

Wänke, M. (2002). Conversational norms and the interpretation of vague quantifiers. Applied Cognitive Psychology, 16(3), 301-307. https://doi.org/10.1002/acp.787

Wagner, L., Holenstein, M., Wepf, H., \& Ruch, W. (2020). Character strengths are related to students' achievement, flow experiences, and enjoyment in teacher-centered learning, individual, and group work beyond cognitive ability. Frontiers in Psychology, 11, 1324.

https://doi.org/10.3389/fpsyg.2020.01324

Watkins, P. C. (2016). Positive Psychology 101. New York, NY: Springer. https://doi.org/110.1891/9780826126986

Weber, M., Ruch, W., Littman-Ovadia, H., Lavy, S., \& Gai, O. (2013). Relationships among higher-order strengths factors, subjective well-being, and general self-efficacy - The case of Israeli adolescents. Personality and Individual Differences, 55(3), 322-327.

https://doi.org/10.1016/j.paid.2013.03.006

Weijters, B., Geuens, M., \& Schillewaert, N. (2010). The stability of individual response styles. Psychological Methods, 15(1), 96-110. https://doi.org/10.1037/a0018721

Widaman, K. (1993). Common factor analysis versus principal component analysis: Differential bias in representing model parameters? Multivariate Behavioral Research, 28(3), 263-311. https://doi.org/10.1207/s15327906mbr2803_1

Wu, A. D., Li, Z., \& Zumbo, B. D. (2007). Decoding the meaning of factorial invariance and updating the practice of multigroup confirmatory factor analysis: A demonstration with TIMSS data. Practical Assessment, Research and Evaluation, 12(3), 1-26. https://doi.org/10.7275/mhqa-cd89 Ziegler, M. (2014). Stop and state your intentions! Let's not forget the ABC of test construction. European Journal of Psychological Assessment, 30(4), 239-242. https://doi.org/10.1027/1015$5759 / \mathrm{a} 000228$ 
Ziegler, M., \& Bensch, D. (2013). Lost in translation: Thoughts regarding the translation of existing psychological measures into other languages. European Journal of Psychological Assessment, 29(2), 81-83. https://doi.org/ 10.1027/1015-5759/a000167

Ziegler, M., Kemper, C. J., \& Kruyen, P. (2014). Short scales - Five misunderstandings and ways to overcome them. Journal of Individual Differences, 35(4), 185-189.

https://doi.org/10.1027/1614-0001/a000148 
HUMAN CHARACTER IN THE IPIP

Tables 
Table 1

VIA Classification of Character Strengths and Associated Virtue Clusters

\begin{tabular}{|c|c|c|}
\hline IPIP-VIA Scale Label & Definition of Strength & Virtue Cluster \\
\hline Appreciation of Beauty \& Excellence [APP] & Recognizing and valuing beauty in the physical environment, skill/talent, or virtues & Transcendence \\
\hline (Capacity for) Love [CAP] & Ability to express warmth and receive love from others, valuing reciprocal caring and sharing & Humanity \\
\hline Citizenship/Teamwork [CIT] & Supporting a group to which one is loyal and contributing to its cause by collaborating and cooperating effectively & Justice \\
\hline Curiosity [CUR] & Desire to explore the world and seek out exciting experiences and novel ideas & Wisdom \\
\hline Equity [EQU] & Treating people equally, with respect and in a fair and unbiased manner & Justice \\
\hline Forgiveness [FOR] & Readily accepting the shortcomings of others and showing mercy & Temperance \\
\hline Gratitude [GRA] & Recognizing good things in life, feeling appreciation, and expressing thanks & Transcendence \\
\hline Hope [HOP] & Seeing a positive future and developing a way to achieve desired events and outcomes & Transcendence \\
\hline Humor [HUM] & Enjoying laughter and bringing smiles to others by highlighting the funny or lighter side of life & Transcendence \\
\hline Industry/Perseverance [IND] & Steadfastness in pursuing goals even in the face of obstacles, [taking pleasure in] accomplishing tasks & Courage \\
\hline Integrity/Honesty [INT] & Being truthful to others and to oneself, trustworthy, and acting with moral integrity & Courage \\
\hline Judgment [JUD] & Weighing all available evidence fairly and being willing to change one's opinion accordingly & Wisdom \\
\hline Kindness $[\mathrm{KIN}]$ & Being concerned about others, helping generously without expecting reciprocity & Humanity \\
\hline Leadership [LEA] & Organizing collective success of a group and fostering good working relationships among members & Justice \\
\hline (Love of) Learning [LOV] & [Having joy when] expanding existing knowledge and learning new skills & Wisdom \\
\hline Modesty [MOD] & Being modest (though realistic) about one's abilities and weaknesses, valuing contributions by others & Temperance \\
\hline Originality/Creativity [ORI] & Having original ideas, coming up with innovative and productive ways to do things & Wisdom \\
\hline Perspective [PER] & Being able to see the big picture and in a position to give good advice on essential questions in life & Wisdom \\
\hline Prudence $[\mathrm{PRU}]$ & Being smart and careful about choices in the interest of avoiding undue risks and preventing regrets & Temperance \\
\hline Self-Regulation [SEL] & Regulating emotions, thoughts, and behaviors to live up to stringent (self-imposed) standards of self-control & Temperance \\
\hline Social Intelligence $[\mathrm{SOC}]$ & Understanding own and other's feelings and motives, knowing how to adapt socially to situations & Humanity \\
\hline Spirituality/Religiousness [SPI] & Seeing a higher purpose and meaning in life, feeling connected to the sacred or believing in transcendent powers & Transcendence \\
\hline Valor/Bravery [VAL] & Conquering fear and standing up for one's convictions in defiance of well-known risks & Courage \\
\hline Zest/Vitality [ZES] & Approaching activities with physical and mental vitality, feeling alive and energetic & Courage \\
\hline
\end{tabular}

Note. IPIP-VIA = Scale labels; definitions adapted from Peterson \& Seligman (2004) and Watkins (2016); Virtue Cluster = Strength association with one of six VIA virtue clusters. 


\section{Table 2a}

Facets Aligned with the Hierarchical Structure Yielding the Big Five Domains (sensu BFI-2)

\begin{tabular}{|c|c|c|}
\hline Trait Label & Definition of Facet & Domain-Facet Abbreviation \\
\hline \multicolumn{3}{|l|}{ Extraversion } \\
\hline Sociability & Desire to socially approach and engage with others & E-s \\
\hline Assertiveness & Willingness to express personal opinions and goals in social situations & $\mathrm{E}-\mathrm{a}$ \\
\hline Energy Level & Positive affect (especially positively aroused states: enthusiasm and excitement) and physical activity level & E-e \\
\hline \multicolumn{3}{|l|}{ Agreeableness } \\
\hline Compassion & Active emotional concern for others' well-being & A-c \\
\hline Respectfulness & Treating others with regard for personal preferences and rights, inhibiting antagonistic/ aggressive impulses & A-r \\
\hline Trust & Holding positive generalized beliefs about others & A-t \\
\hline \multicolumn{3}{|l|}{ Conscientiousness } \\
\hline Organization & Preference for order and structure & C-o \\
\hline Productiveness & Work ethic and persistence while pursuing goals & $C-p$ \\
\hline Responsibility & Commitment to meeting duties and obligations & C-r \\
\hline \multicolumn{3}{|l|}{ Negative Emotionality } \\
\hline Anxiety & Tendency to experience anxiety and fear & $\mathrm{N}-\mathrm{a}$ \\
\hline Depression & Tendency toward depression and sadness & $N-d$ \\
\hline Emotional Volatility & Volatile mood swings & $\mathrm{N}-\mathrm{e}$ \\
\hline \multicolumn{3}{|l|}{ Oppen-Mindedness } \\
\hline Intellectual Curiosity & Intellectual interests and enjoyment of thinking & O-i \\
\hline Aesthetic Sensitivity & Broader alternative primarily defined by intellectual and artistic interests (+ other characteristics) & $0-\mathrm{a}$ \\
\hline Creative Imagination & Creativity and originality & O-c \\
\hline
\end{tabular}

Note. Table based on Soto \& John (2017a). Trait Label = Big Five domain (bold) or facet name (Negative Emotionality is historically known as Neuroticism or, reverse-coded Emotional Stability, and Open-Mindedness as Openness to [cognitive] experience); Domain = Big Five domain associated with a facet. The first facet for each domain is factorpure, each next pair presents complementary facets (Open-Mindedness facets do not strictly conform to this rule). Underlining reflects letters used for acronyms for Big Five domain and facet names. 


\section{Table 2b}

Definitions of Basic Human Values and Associated Value Clusters

\begin{tabular}{|c|c|c|}
\hline Value Label & Definition of Value & Value Cluster \\
\hline Power & $\begin{array}{l}\text { Social status and prestige, control or dominance over people and resources (authority, social power, wealth, } \\
\text { preserving my public image) }\end{array}$ & Self-Ennhancement \\
\hline Achievement & $\begin{array}{l}\text { Personal success through demonstrating competence according to social standards (ambitious, successful, } \\
\text { capable, influential) }\end{array}$ & Self-Énhancement \\
\hline Hedonism & Pleasure or sensuous gratification for oneself (pleasure, enjoying life, self-indulgent) & Openness to $\underline{\text { Change }}$ \\
\hline Stimulation & Excitement, novelty, and challenge in life (daring, a varied life, an exciting life) & Openness to $\underline{\text { Change }}$ \\
\hline Self-Direction & $\begin{array}{l}\text { Independent thought and action-choosing, creating, exploring (creativity, freedom, independent, choosing } \\
\text { own goals, curious) }\end{array}$ & Openness to $\underline{\text { Change }}$ \\
\hline Universalism & $\begin{array}{l}\text { Understanding, appreciation, tolerance, and protection for the welfare of all people and for nature (equality, } \\
\text { social justice, wisdom, broadminded, protecting the environment, unity with nature, a world of beauty) }\end{array}$ & Self-Iranscendence \\
\hline Benevolence & $\begin{array}{l}\text { Preservation and enhancement of the welfare of people with whom one is in frequent personal contact } \\
\text { (helpful, honest, forgiving, loyal, responsible) }\end{array}$ & Self-Transcendence \\
\hline Tradition & $\begin{array}{l}\text { Respect, commitment, and acceptance of the customs and ideas that traditional culture or religion provide } \\
\text { (devout, respect for tradition, humble, moderate) }\end{array}$ & Conservation \\
\hline Conformity & $\begin{array}{l}\text { Restraint of actions, inclinations, and impulses likely to upset or harm others and violate social expectations } \\
\text { or norms (self-discipline, politeness, honoring parents and elders, obedience) }\end{array}$ & Conservation \\
\hline Security & $\begin{array}{l}\text { Safety, harmony, and stability of society, of relationships, and of self (family security, national security, social } \\
\text { order, clean, reciprocation of favors) }\end{array}$ & Conservation \\
\hline
\end{tabular}

Note. Table adapted from Schwartz \& Boehnke (2004, Table 2). Value label = Basic human value sensu Schwartz; Value Cluster = Higher-order dimension pole. Underlining reflects acronyms for value cluster names. 
Table 3

IPIP-VIA Item and Scale Adaptations During Revision and TRAPD Approach

\begin{tabular}{|c|c|c|c|}
\hline IPIP-VIA Scale Adaptations & Occurrences at Item Level & Occurrences at Scale Level & $\%$ Scales Affected \\
\hline \multicolumn{4}{|l|}{ Item Numbers } \\
\hline Considered IPIP-VIA items & 252 & 24 & 100.0 \\
\hline Dropped IPIP-VIA items before administration & 156 & 24 & 100.0 \\
\hline Identified alternative (non-VIA) IPIP items & 126 & 23 & 95.8 \\
\hline Tested (non-VIA) IPIP items & 7 & 7 & 29.2 \\
\hline Preferred (non-VIA) IPIP items & 6 & 6 & 25.0 \\
\hline Tested item variants ( $A$ vs. $B$ ) in UK \& DE & 5 & 5 & 20.8 \\
\hline Preferred item variants $B$ over $A$ in UK \& DE & 3 & 3 & 12.5 \\
\hline Tested additional translation variants ( $A$ vs. $B$ ) in $D E$ & 5 & 4 & 16.7 \\
\hline Preferred additional translation variants $B$ over $A$ in $D E$ & 5 & 4 & 16.7 \\
\hline \multicolumn{4}{|l|}{$\underline{\text { Item Alterations }}$} \\
\hline Level 0: Translation straightforward & 34 & 22 & 32.1 \\
\hline Level 1: Translation linguistically complex & 38 & 22 & 35.8 \\
\hline Level 2: Adaptation culture-related & 0 & 0 & 0.0 \\
\hline Level 3: Adaptation measurement-related & 12 & 10 & 11.3 \\
\hline Level 4: Adaptation construct-related & 22 & 12 & 20.8 \\
\hline Other item alterations & 0 & 0 & 0.0 \\
\hline \multicolumn{4}{|l|}{$\underline{\text { Response Scale Alterations }}$} \\
\hline Changed number of response categories & 0 & 0 & 0.0 \\
\hline Changed scale anchors (response labels) & 96 & 24 & 100.0 \\
\hline Other Adaptations & 0 & 0 & 0.0 \\
\hline
\end{tabular}

Note: Percentages do not sum to $100 \%$ due to multiple adaptations of the scales. 
Table 4

Socio-demographic Descriptives of Samples 1-5

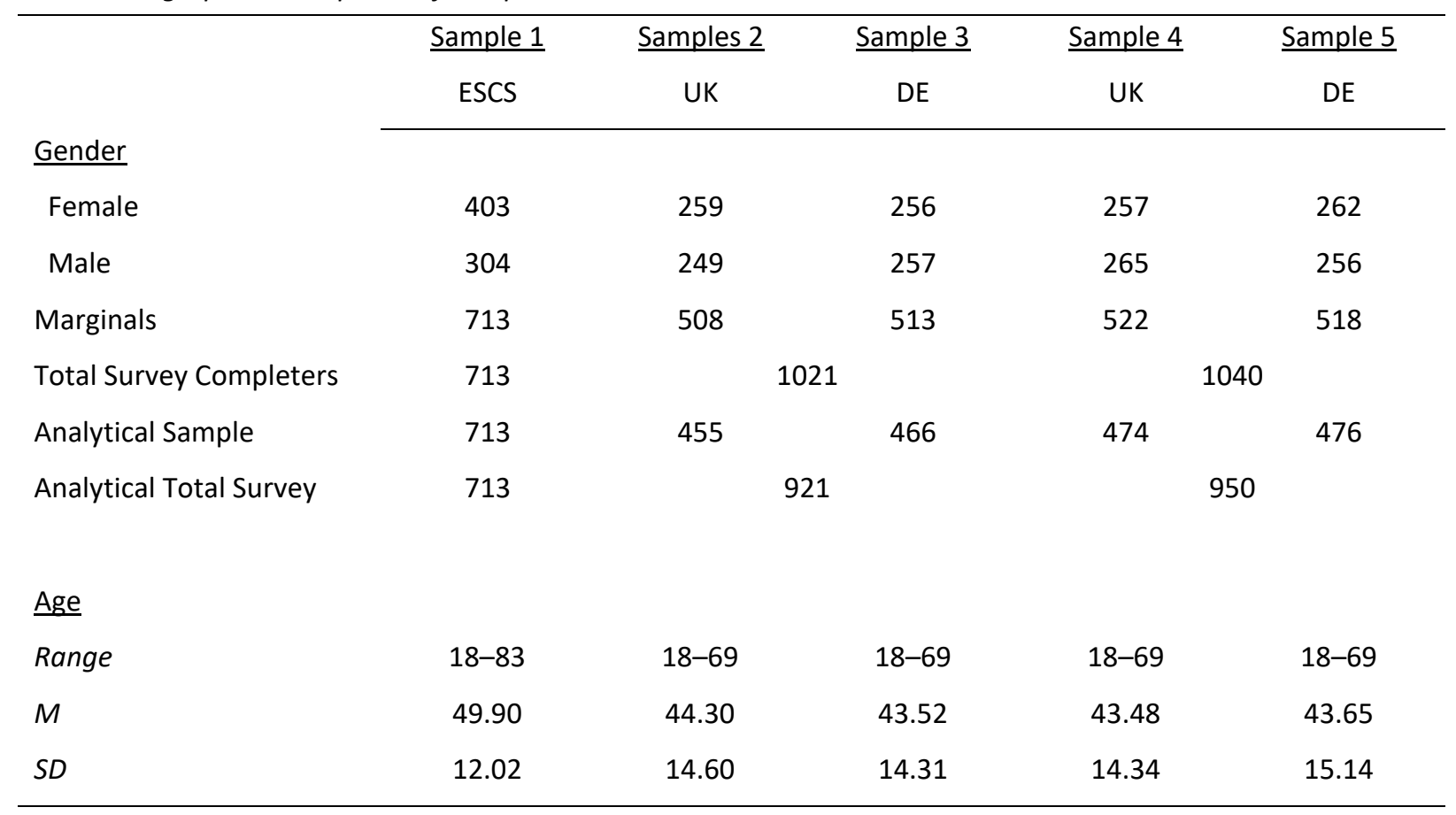

Note. Samples 1-5 = available survey completers only. ESCS = Eugene-Springfield Community Sample (six gen-

der values missing); UK = United Kingdom; DE = Germany; Analytical Sample = after filtering out participants for poor data quality (see text). Samples 2 and 3 (IPIP-VIA-R Version A) comprised 7 missing data points, Samples 4 and 5 (IPIP-VIA-R Version B) comprised 2 missing data points. 
Table 5

Cutoff Criteria for Factor Analytical Procedures in Scale Development (Subject to Professional Judgment)

\begin{tabular}{l}
\hline Index (Short- Index Definition and Relevance \\
hand) \\
to Short Scales \\
(IPIP-VIA-R)
\end{tabular}

Suitability of Factor Analysis (EFA, PCA)

KMO Kaiser-Meyer-Olkin criterion of overall sampling adequacy: suitability of a data matrix prior to any factoring procedures

Unidimensionality

ECV Explained Common Variance: variance explained by first (common) Minimum Rank Factor Analysis factor divided by the total common variance, i.e., the dominance of the first factor over other factors (or relative general-factor strength index)

MIREAL Mean Item Residual Absolute Loading: average of item loadings on secondary factor

Factor Reliability \& Construct Replicability

FDI Factor Determinacy Index: correlation between factor scores and intended factor (congeneric latent variable)

$\mathrm{H} / \mathrm{gHI} \quad \mathrm{H}$-Index: proportion of variance explained by congeneric latent variable indicators (Hancock \& Mueller, 2001), also termed maximal reliability (internal consistency for an optimally weighted scaling procedure)

$\mathrm{gHI}$ is the generalized $\mathrm{H}$-Index applicable to categorical data, also in the presence of secondary (even oblique) factors; it may refer to
KMO < .50 ("unacceptable") - Kaiser \& Rice (1974)

$\mathrm{KMO} \geq .60-$ Dziuban \& Shirkey (1974)

$\mathrm{KMO}=.60$ ("mediocre") - Lorenzo-Seva \& Ferrando (2013)

$\mathrm{KMO} \geq .60-$ Tabachnick \& Fidell (2013)

ECV $\geq .70-.85$ ("essentially unidimensional") - Ferrando \& Lorenzo-Seva (2018), following Green et al. (1984) and Rodriguez et al. (2016)

MIREAL $\leq .30$ ("rough initial reference" for essential unidimensionality) - Ferrando \& Lorenzo-Seva (2018), following Grice (2001)

Strength of loading indicating a factor: .32 ("poor"), .45 ("fair"), .55 ("good"), .63 ("very good") or .71 ("excellent") - Tabachnick \& Fidell (2013), following Comrey \& Lee (1992)

FDI $\geq .80$ ("adequate") - Gorsuch (1983)

FDI $\geq .90$ ("recommended") - Brown (2015), Beauducel (2011)

$\mathrm{H} \geq .70$ ("reasonable") - Hancock \& Mueller (2001)

$\mathrm{H} \geq .80$ ("well-defined latent variable") - Rodriguez et al. (2016)

gH latent $\geq .80$ ("reasonable") - Ferrando \& Lorenzo-Seva (2018)
$\mathrm{FDI} \geq .80$

$\mathrm{KMO} \geq .60$

MIREAL $\leq .40$

gHl $_{\text {observed }} \geq .65$ 
latent response variables or observed variables (Ferrando \& Lorenzo-Seva, 2018); the expected gHI values are lower for observed than for latent response variables (the latter are merely hypothetical)

\section{Scale Reliability}

AVE

Average Variance Extracted: average ratio of item variance explained by the respective construct (and identified secondary sources of variability), as a conservative approach to the overall validity of a measurement model (also referred to as "convergent validity" from the perspective of the indicators)

$\omega($ or $\mathrm{CR})$

Omega: proportion of variance in the unit-weighted scale score attributable to the common factor (but not group-factors or item-specific factors) hence a measure of general factor saturation (McDonald, 1999), also termed Composite Reliability (Fornell \& Larcker, 1981) or Raykov's (1997) rho; unlike Cronbach's $\alpha, \omega$ does not depend on the assumption of essential tau-equivalence and is unbiased if multidimensional items are properly specified

Test-retest reliability: rank-order consistency (not: repeatability or agreement of successive measurements)

\section{Model Fit}

$\chi^{2} \mathrm{SB}$

Chi-square statistic: Satorra-Bentler scaled for using robust ML (MLR), with $d f$ being the expected value

$\chi^{2}$ sв / $d f \quad$ Normed chi-square: Ratio of chi-square to degrees of freedom

CFI Comparative Fit Index: incremental measure of fit in comparison to null model (Bentler, 1990)

RMSEA Root Mean Square Error of Approximation: measure of approximate fit based on the discrepancy per degree of freedom (Steiger, 1990)
AVE $\geq .50$ - Fornell \& Larker (1981)

$\mathrm{AVE} \leq .50$ if:

AVE $<.50$ ("acceptable") if composite reliability is high enough:

- CR $\geq .60$ - Fornell \& Larcker (1981)

- CR $\geq .70$ - Hair et al. (2006).

Reliability norms for $\omega \approx \alpha$

$\alpha \geq .90$ ("excellent"), .80 ("good"), .70 ("acceptable"), .60 ("ques-

tionable"), or .50 ("poor") - Nunnally \& Bernstein (1994)

$\alpha \approx .70-$ Schmitt (1996) denied that $\alpha$ must always exceed .70

$r_{\mathrm{tt}}:$ cutoff is "circumstantial" - Crocker \& Algina (1986)

$r_{\mathrm{tt}} \geq .80$ (assuming character strengths are stable traits)

nonsignificant

$\chi^{2}$ sв $/ d f \leq 2-3-$ Kline (2011)

$\chi^{2} \mathrm{SB} / d f \leq 5-$ Schumacker \& Lomax (2004)

CFI $\geq .95-\mathrm{Hu} \&$ Bentler (1999)

CFI $<.90$ ("poor fit") - Bentler \& Bonett (1980)

RMSEA $\leq .06-$ Hu \& Bentler (1999)

RMSEA $\leq .10$ (“mediocre") - Browne \& Cudeck (1993) $r_{\mathrm{tt}}>.70$

$\mathrm{CR}(=\omega) \geq .60$

$\omega \geq .70$

nonsignificant

$\chi^{2} \mathrm{sB} / d f \leq 10$

$\mathrm{CFI} \geq .90$

RMSEA $\leq .10$ 
SRMR

Standardized Root Mean Square Residual: discrepancy between the standardized sample covariance matrix and the model covariance matrix (Hu \& Bentler, 1999); alternatively: discrepancy between the observed and estimated correlations, standardized means, and variances (Asparouhov \& Muthén, 2018)

$\mathrm{BIC}$
SRMR $\leq .05$ (Byrne, 1998)

SRMR $\leq .08$ (Hu \& Bentler, 1999)
$\mathrm{SRMR} \leq .08$

smaller comparisons

Note. Instead of using Mplus's CFI and RMSEA (or scaled versions) for evaluating the initial measurement models, we relied on the nonnormality-robust, sample-appropriate indices that reflect true population characteristics: CFI robust and RMSEA robust (Brosseau-Liard \& Savalei, 2014; Brosseau-Liard, Savalei, \& Li, 2012), which at the time of the study, were available only from R/lavaan. RMSEArobust will usually be higher than RMSEAscaled or RMSEA, whereas CFIrobust can be higher or lower than CFI Iscaled or CFI (Savalei, 2018). For invariance testing though, we used Mplus's $\mathrm{CFI}_{\text {scaled }}$ and RMSEA scaled for, because the prominent cut-off heuristics for inspecting model differences to determine nonequivalence

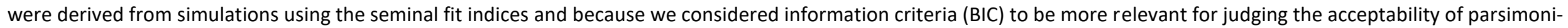
ous measurement invariance models anyway. 
Table 6

IPIP-VIA Dimensionality in Full (Sample 1) and Abbreviated IPIP-VIA Strength Scales (Variant A for Samples 2 and 3; Variants A/B for Samples 4 and 5)

\begin{tabular}{|c|c|c|c|c|c|c|c|c|c|c|c|c|c|c|c|}
\hline \multirow[t]{3}{*}{ Scale } & \multicolumn{3}{|c|}{ Full Scale: Sample 1} & \multicolumn{6}{|c|}{ Abbreviated Scale Variant A: Samples 2 and 3} & \multicolumn{6}{|c|}{ Abbreviated Scale Variant A/B: Samples 4 and 5} \\
\hline & \multirow{2}{*}{$\begin{array}{c}\text { MAP-Test } \\
\text { ESCS }\end{array}$} & \multirow{2}{*}{$\begin{array}{c}\text { PA-PCA } \\
\text { ESCS } \\
\end{array}$} & \multirow{2}{*}{$\begin{array}{c}\text { PA-PAF } \\
\text { ESCS }\end{array}$} & \multicolumn{2}{|c|}{ PA-MRFA } & \multicolumn{2}{|c|}{ ECV } & \multicolumn{2}{|c|}{ MIREAL } & \multicolumn{2}{|c|}{ PA-MRFA } & \multicolumn{2}{|c|}{ ECV } & \multicolumn{2}{|c|}{ MIREAL } \\
\hline & & & & UK & $\mathrm{DE}$ & UK & $\mathrm{DE}$ & UK & $\mathrm{DE}$ & UK & $\mathrm{DE}$ & UK & $\mathrm{DE}$ & UK & $\mathrm{DE}$ \\
\hline APP & 1 & 3 & 3 & 0 & 0 & .53 & .81 & .48 & .19 & $0 / 0$ & $0 / 1$ & $.54 / .63$ & $.83 / .74$ & $.46 / .42$ & $.18 / .29$ \\
\hline CAP & 1 & 1 & 2 & 0 & 1 & .71 & .81 & .32 & .24 & 1 & 1 & .73 & .82 & .33 & .27 \\
\hline CIT & 1 & 2 & 2 & 1 & 0 & .70 & .65 & .41 & .33 & 2 & $0 / 1$ & .70 & $.63 / .68$ & .38 & $.41 / .34$ \\
\hline CUR & 1 & 2 & 2 & 1 & 1 & .74 & .76 & .36 & .34 & 1 & 1 & .73 & .76 & .34 & .33 \\
\hline EQU & 1 & 1 & 3 & 1 & 0 & .74 & .82 & .35 & .20 & 1 & $1 / 1$ & .77 & $.79 / .76$ & .34 & $.26 / .30$ \\
\hline FOR & 1 & 1 & 3 & 1 & 1 & .69 & .77 & .37 & .30 & 0 & $1 / 1$ & .67 & $.71 / .84$ & .37 & $.37 / .24$ \\
\hline GRA & 1 & 1 & 3 & 0 & 1 & .68 & .73 & .40 & .32 & 1 & 1 & .69 & .79 & .41 & .26 \\
\hline HOP & 1 & 2 & 2 & 1 & 0 & .69 & .67 & .41 & .38 & 0 & 1 & .60 & .69 & .48 & .40 \\
\hline HUM & 1 & 1 & 4 & 1 & 1 & .72 & .82 & .34 & .31 & 0 & 1 & .68 & .81 & .40 & .29 \\
\hline IND & 1 & 1 & 3 & 1 & 1 & .78 & .85 & .32 & .28 & 1 & 1 & .76 & .94 & .35 & .15 \\
\hline INT & 1 & 2 & 3 & 1 & 1 & .77 & .81 & .34 & .27 & 1 & 1 & .73 & .88 & .39 & .23 \\
\hline JUD & 1 & 2 & 3 & 1 & 0 & .70 & .67 & .35 & .34 & 0 & 1 & .66 & .72 & .41 & .31 \\
\hline KIN & 1 & 1 & 3 & 1 & 1 & .67 & .83 & .37 & .25 & 2 & 1 & .69 & .70 & .39 & .33 \\
\hline LEA & 1 & 2 & 3 & 1 & 1 & .75 & .79 & .35 & .30 & 0 & 1 & .63 & .75 & .40 & .33 \\
\hline LOV & 2 & 2 & 2 & 0 & 1 & .63 & .75 & .34 & .31 & $0 / 1$ & $1 / 1$ & $.63 / .78$ & $.71 / .89$ & $.34 / .31$ & $.33 / .17$ \\
\hline MOD & 1 & 2 & 4 & 0 & 1 & .64 & .81 & .37 & .24 & 0 & 0 & .62 & .70 & .43 & .31 \\
\hline ORI & 1 & 1 & 2 & 1 & 1 & .75 & .86 & .37 & .23 & 1 & 1 & .69 & .81 & .42 & .28 \\
\hline PER & 1 & 2 & 4 & 1 & 1 & .70 & .72 & .35 & .34 & 0 & 1 & .67 & .70 & .40 & .35 \\
\hline PRU & 1 & 3 & 5 & 0 & 1 & .61 & .72 & .42 & .35 & $0 / 1$ & $1 / 0$ & $.59 / .73$ & $.73 / .71$ & $.41 / .30$ & $.32 / .27$ \\
\hline SEL & 1 & 2 & 4 & 0 & 1 & .60 & .71 & .38 & .26 & 0 & $0 / 0$ & .59 & $.61 / .65$ & .40 & $.34 / .32$ \\
\hline SOC & 1 & 2 & 4 & 0 & 1 & .66 & .76 & .40 & .30 & 1 & 0 & .67 & .69 & .43 & .36 \\
\hline SPI & 1 & 1 & 3 & 0 & 0 & .60 & .67 & .42 & .44 & $0 / 0$ & $1 / 0$ & $.66 / .64$ & $.73 / .64$ & $.38 / .39$ & $.37 / .35$ \\
\hline VAL & 1 & 2 & 4 & 0 & 1 & .66 & .90 & .38 & .18 & 0 & 1 & .66 & .86 & .40 & .20 \\
\hline ZES & 1 & 1 & 3 & 1 & 1 & .64 & .77 & .37 & .33 & $0 / 1$ & $1 / 1$ & $.68 / .67$ & $.77 / .75$ & $.40 / .39$ & $.33 / .35$ \\
\hline
\end{tabular}

Note: MAP-Test = Velicer's Minimum Average Partial Test, PA-PCA: Parallel Analysis based on Principal Component Analysis; PA-PAF: Parallel Analysis based on Principal Axis Factor Analysis; PA-MRFA: Parallel Analysis based on Minimum Rank Factor Analysis; ECV: Common Variance explained by first factor; MIREAL: Mean Item Residual Average Loading. 
Table 7

CFA Model Fit \& Factor Loadings: IPIP-VIA-R - Final IPIP-VIA-R Scales (Samples 4 and 5)

\begin{tabular}{|c|c|c|c|c|c|c|c|c|c|c|c|c|c|c|c|c|c|c|}
\hline & \multicolumn{2}{|c|}{$\chi^{2} \mathrm{sB}(d f=5)$} & \multicolumn{2}{|c|}{ P-Value } & \multicolumn{2}{|c|}{$\mathrm{CFI}$} & \multicolumn{2}{|c|}{ RMSEA } & \multicolumn{2}{|c|}{ SRMR } & \multicolumn{2}{|c|}{$\left|\lambda_{1}\right|$} & \multicolumn{2}{|c|}{$\left|\lambda_{2}\right|$} & \multicolumn{2}{|c|}{$\left|\lambda_{3}\right|$} & \multicolumn{2}{|c|}{$\left|\lambda_{4}\right|$} \\
\hline & UK & $\mathrm{DE}$ & UK & $\mathrm{DE}$ & UK & $\mathrm{DE}$ & UK & DE & UK & $\mathrm{DE}$ & UK & $\mathrm{DE}$ & UK & $\mathrm{DE}$ & UK & $\mathrm{DE}$ & UK & $\mathrm{DE}$ \\
\hline $\mathrm{APP}_{\mathrm{B}}$ & 8.54 & 6.00 & .13 & .31 & .99 & 1.00 & .04 & .02 & .04 & .04 & .37 & .53 & .54 & .53 & .64 & .63 & .56 & .45 \\
\hline CAP & 12.55 & 24.52 & .03 & .00 & .98 & .94 & .06 & .09 & .04 & .06 & .42 & .57 & .46 & .36 & .60 & .67 & .77 & .64 \\
\hline $\mathrm{CIT}_{\mathrm{B}}$ & 9.49 & 25.22 & .09 & .00 & .99 & .90 & .05 & .11 & .04 & .11 & .53 & .58 & .45 & .46 & .78 & .53 & .41 & .45 \\
\hline CUR & 5.24 & 10.30 & .39 & .07 & 1.00 & .99 & .01 & .06 & .04 & .05 & .42 & .53 & .66 & .72 & .51 & .54 & .56 & .78 \\
\hline $\mathrm{EQU}_{\mathrm{B}}$ & 6.60 & 8.30 & .25 & .14 & 1.00 & .99 & .03 & .05 & .03 & .05 & .66 & .73 & .41 & .43 & .77 & .60 & .47 & .62 \\
\hline $\mathrm{FOR}_{\mathrm{B}}$ & 12.47 & 15.73 & .03 & .01 & .99 & .98 & .06 & .07 & .05 & .07 & .37 & .55 & .68 & .75 & .41 & .41 & .67 & .84 \\
\hline GRA & 7.83 & 26.71 & .17 & .00 & 1.00 & .93 & .04 & .10 & .04 & .10 & .70 & .45 & .65 & .61 & .61 & .55 & .41 & .62 \\
\hline HOP & 53.67 & 41.92 & .00 & .00 & .95 & .93 & .12 & .12 & .05 & .06 & .72 & .71 & .52 & .50 & .57 & .55 & .48 & .63 \\
\hline HUM & 10.00 & 5.60 & .08 & .35 & .99 & 1.00 & .05 & .02 & .03 & .03 & .41 & .64 & .69 & .72 & .61 & .72 & .59 & .57 \\
\hline IND & 3.71 & 16.79 & .59 & .00 & 1.00 & .97 & .00 & .08 & .02 & .06 & .41 & .53 & .63 & .75 & .73 & .72 & .63 & .60 \\
\hline INT & 9.67 & 13.84 & .09 & .02 & .99 & .97 & .05 & .08 & .05 & .10 & .65 & .66 & .68 & .69 & .51 & .47 & .59 & .57 \\
\hline JUD & 11.87 & 11.97 & .04 & .04 & .99 & .98 & .06 & .05 & .04 & .06 & .48 & .39 & .60 & .55 & .62 & .61 & .42 & .45 \\
\hline KIN & 11.56 & 16.55 & .04 & .01 & .99 & .95 & .05 & .08 & .04 & .08 & .62 & .58 & .57 & .53 & .61 & .59 & .43 & .36 \\
\hline LEA & 25.60 & 27.76 & .00 & .00 & .97 & .95 & .09 & .09 & .05 & .04 & .53 & .57 & .67 & .72 & .59 & .56 & .41 & .55 \\
\hline $\mathrm{LOV}_{\mathrm{B}}$ & 12.49 & 6.62 & .03 & .25 & .99 & 1.00 & .06 & .03 & .05 & .03 & .70 & .45 & .30 & .33 & .56 & .79 & .67 & .76 \\
\hline MOD & 28.38 & 14.58 & .00 & .01 & .95 & .95 & .10 & .07 & .05 & .05 & .42 & .37 & .56 & .67 & .57 & .58 & .49 & .38 \\
\hline ORI & 27.88 & 6.84 & .00 & .23 & .97 & 1.00 & .09 & .03 & .05 & .03 & .44 & .60 & .62 & .61 & .65 & .74 & .58 & .59 \\
\hline PER & 5.94 & 7.17 & .31 & .21 & 1.00 & .99 & .02 & .03 & .04 & .04 & .64 & .49 & .50 & .66 & .48 & .49 & .55 & .59 \\
\hline $\mathrm{PRU}_{\mathrm{A}}$ & 9.73 & 22.16 & .08 & .00 & .99 & .96 & .04 & .09 & .04 & .07 & .60 & .72 & .31 & .33 & .48 & .65 & .46 & .59 \\
\hline $\mathrm{SEL}_{B}$ & 8.96 & 13.12 & .11 & .02 & .99 & .97 & .04 & .05 & .04 & .05 & .31 & .35 & .25 & .45 & .70 & .55 & .61 & .42 \\
\hline SOC & 15.93 & 5.94 & .01 & .31 & .98 & 1.00 & .07 & .02 & .05 & .03 & .51 & .31 & .61 & .55 & .64 & .72 & .61 & .53 \\
\hline $\mathrm{SPI}_{\mathrm{A}}$ & 82.58 & 52.97 & .00 & .00 & .84 & .89 & .17 & .16 & .08 & .07 & .39 & .79 & .61 & .44 & .71 & .38 & .37 & .80 \\
\hline VAL & 2.60 & 20.40 & .76 & .00 & 1.00 & .96 & .00 & .09 & .03 & .07 & .43 & .64 & .43 & .42 & .61 & .75 & .61 & .52 \\
\hline ZES $_{B}$ & 8.51 & 17.70 & .13 & .00 & 1.00 & .97 & .04 & .08 & .03 & .07 & .67 & .76 & .37 & .45 & .76 & .75 & .55 & .57 \\
\hline Mean & - & - & .14 & .08 & .98 & .96 & .06 & .07 & .04 & .06 & .52 & .56 & .53 & .55 & .61 & .61 & .54 & .58 \\
\hline
\end{tabular}

Note. $N=950$ (474 and 476 in UK and DE, respectively). The presence of a scale name index indicates the item/scale variant tested. CFI = (robust) Comparative Fit Index; RMSEA = (robust) Root Mean Square Error of Approximation; SRMR = Standardized Root Mean Square Residual; $\left|\lambda_{1}\right| . . .\left|\lambda_{4}\right|=$ factor loadings (standardized regression weights), with loading sign reversed for negatively keyed items. 


\section{Table 8}

Original English and German Adaptations of IPIP-VIA-R Items [including (revised) Scale Labels]

\begin{tabular}{|c|c|c|c|}
\hline Pos. & Key & English Items (IPIP Variable No.) & German Items \\
\hline & & Appreciation of Beauty and Excellence [APP] & Sinn für das Schöne und Exzellenz [APP] \\
\hline 4 & - & Am rarely aware of the natural beauty in the environment. (v208) & Ich mache mir die Schönheit der Natur nur selten bewusst. \\
\hline 28 & + & Experience deep emotions when I see beautiful things. (v102) & Wenn ich etwas Schönes sehe, bewegt mich das sehr. \\
\hline 52 & - & Fail to notice beauty until other comment on it. (v139) & Ich bemerke Schönheit erst dann, wenn andere darauf aufmerksam machen. \\
\hline \multirow[t]{2}{*}{76} & + & Feel it's important to live in a world of beauty. (v52) & Ich spüre, dass es wichtig ist, in einer Welt voller Schönheit zu leben. \\
\hline & & (Capacity for) Love [CAP] - rev: Love [LOV] & Liebesfähigkeit [CAP] - rev: Fähigkeit zu lieben und geliebt zu werden [LOV] \\
\hline 3 & - & Do not easily share my feelings with others. (v13) & Es fällt mir schwer, anderen meine Gefühle zu zeigen. \\
\hline 27 & + & $\begin{array}{l}\text { Know that there are people in my life who care as much for me as for } \\
\text { themselves. (v55) }\end{array}$ & $\begin{array}{l}\text { Ich bin mir gewiss, dass es Menschen in meinem Leben gibt, denen mein Wohl } \\
\text { genauso wichtig ist wie ihr eigenes. }\end{array}$ \\
\hline 51 & + & Can express love to someone else. (v234) & Ich bin fähig, meine Zuneigung anderen gegenüber auszudrücken. \\
\hline \multirow[t]{2}{*}{75} & - & Have difficulty accepting love from anyone. (v340) & Es fällt mir grundsätzlich schwer, Liebe anzunehmen. \\
\hline & & Citizenship [CIT] - rev: Teamwork [TEA] & Soziale Verantwortlichkeit [CIT] - rev: Teamfähigkeit [TEA] \\
\hline 6 & - & Am not good at working with a group. (v16) & Ich kann einfach nicht gut mit anderen zusammenarbeiten. \\
\hline 30 & + & Am an extremely loyal person. (v92) & Ich bin sehr loyal, verlässlich. \\
\hline 54 & + & Support my teammates or fellow group members. (v236) & Ich setze mich für mein Team oder eine größere Gemeinschaft ein. \\
\hline \multirow[t]{2}{*}{78} & - & Prefer to do everything alone. (v73) & Ich mache lieber alles alleine. \\
\hline & & Curiosity [CUR] & Neugier [CUR] \\
\hline 7 & - & Am not all that curious about the world. (v115) & Das Meiste in dieser Welt weckt nicht gerade meine Neugier. \\
\hline 31 & + & Am excited by many different activities. (v151) & Ich kann mich für viele verschiedene Aktivitäten begeistern. \\
\hline 55 & + & Can find something of interest in any situation. (v268) & Ich kann in jeder Situation etwas finden, was mein Interesse weckt. \\
\hline 79 & - & Have few interests. (v342) & Es gibt nur wenige Dinge, die mich wirklich interessieren. \\
\hline
\end{tabular}

Equity [EQU]

$12+\quad$ Treat all people equally. (v94)

Ich behandle alle Menschen gleich fair. 


$\begin{array}{lll}36 & - & \text { Take advantage of others. (v109) } \\ 60 & + & \text { Believe that everyone's rights are equally important. (v120) } \\ 84 & - & \text { Treat others differently if I don't like them. (v241) }\end{array}$

\section{Forgiveness [FOR]}

\begin{tabular}{lll}
5 & + & Try to respond with understanding when someone treats me badly. (v269) \\
29 & - & Hold grudges. (v72) \\
53 & + & Allow others to make a fresh start. (v149) \\
77 & - & Find it hard to forgive others. (X210) \\
\hline
\end{tabular}

\section{Gratitude [GRA]}

$\begin{array}{lll}10 & + & \text { Express my thanks to those who care about me. (v26) } \\ 34 & + & \text { Am an extremely grateful person. (v175) } \\ 58 & - & \text { Feel no gratitude to others. (Q58) } \\ 82 & - & \text { Find few things in my life to be grateful for. (v248) }\end{array}$

\section{Hope [HOP]}

$\begin{array}{lll}21 & - & \text { Expect the worst. (v36) } \\ 45 & + & \text { Can find the positive in what seems negative to others. (v65) } \\ 69 \quad+\quad & \text { Remain hopeful despite challenges. (v106) } \\ 93 \quad-\quad \begin{array}{l}\text { Often think about the possibility of negative outcomes that are not likely } \\ \text { to occur. (D47) }\end{array}\end{array}$

\begin{tabular}{ccl}
\hline & & \multicolumn{1}{c}{ Humor [HUM] } \\
19 & - & Am not known for my sense of humor. (v45) \\
43 & + & Use laughter to brighten the days of others. (v111) \\
67 & - & Am not fun to be with. (v76) \\
91 & + & Keep my sense of humor even in gloomy situations. (v216) \\
\hline
\end{tabular}

Ich tue immer wieder mal Dinge auf Kosten anderer.

Mir ist es wichtig, dass alle Menschen die gleichen Rechte haben.

Wenn ich Leute nicht mag, behandele ich sie schlechter.

\section{Vergebungsbereitschaft [FOR]}

Ich bin nicht nachtragend, wenn mich jemand schlecht behandelt.

Es dauert lange, bis ich verzeihen kann.

Ich gebe anderen immer die Möglichkeit für einen Neuanfang.

Ich kann anderen nicht so leicht vergeben.

\section{Dankbarkeit [GRA]}

Menschen, die sich um mein Wohl sorgen, zeige ich stets meine Dankbarkeit. Ich bin von Dankbarkeit erfüllt.

Anderen gegenüber kann ich echt keine Dankbarkeit empfinden.

Ich finde in meinem Leben nur wenige Dinge, für die ich dankbar sein könnte.

\section{Hoffnung [HOP]}

Ich neige dazu, mit dem Schlimmsten zu rechnen.

Ich kann Gutes selbst dort finden, wo andere nur Schlechtes sehen.

Selbst bei Herausforderungen bleibe ich hoffnungsvoll.

Ich male mir häufig schlimme Dinge aus, die wahrscheinlich nie passieren werden.

\section{Humor [HUM]}

Ich bin nicht gerade für meinen Sinn für Humor bekannt.

Ich heitere andere durch Lachen auf.

Ich bin keine besonders unterhaltsame Person.

Selbst in trostlosen Situationen behalte ich meinen Sinn für Humor.

Ich bleibe an Aufgaben solange dran, bis ich sie erledigt habe.

Ich fange viele Dinge an, bringe sie aber nicht zu Ende. 
$56+\quad$ Finish things despite obstacles in the way. (v126)

$80 \quad-\quad$ Give up easily. (v200)

Integrity/Honesty [INT]

$15+$ Am trusted to keep secrets. (v10)

$39+\quad$ Keep my promises. (v14)

63 - Lie to get myself out of trouble. (v143)

$87 \quad-\quad$ Cheat on people who have trusted me. (Q22)

\section{Judgment [JUD]}

13 - Don't tend to think things through critically. (v118)

37 - Don't think about different possibilities when making decisions. (v25)

$61+$ Weigh the pros and the cons. (v257)

$85+$ Am valued by my friends for my good judgment. (v333)

Kindness [KIN]

9 - $\quad$ Get impatient when others talk to me about their problems. (v6)

$33+\quad$ Am never too busy to help a friend. (v24)

$57+\quad$ Go out of my way to cheer up people who appear down. (v88)

$81 \quad-\quad$ Am only kind to others if they have been kind to me. (v195)

\begin{tabular}{lll}
\hline & & \\
16 & - & Have difficulty getting others to work together. (v54) \\
40 & + & Am good at helping people work well together. (v96) \\
64 & - & Am not good at taking charge of a group. (v105) \\
88 & + & Am told that I am a strong but fair leader. (v329)
\end{tabular}

Ich bringe Dinge zu Ende, auch wenn ich dafür Hindernisse überwinden muss. Oft gebe ich zu schnell auf.

\section{Integrität/Ehrlichkeit [INT]}

Geheimnisse, die man mir anvertraut, behalte ich für mich.

Ich halte meine Versprechen.

Wenn ich mir dadurch Ärger ersparen kann, nehme ich es mit der Wahrheit nicht so genau.

Ich missbrauche das Vertrauen anderer.

\section{Urteilsvermögen [JUD]}

Ich neige nicht dazu, Dinge zu durchdenken und kritisch zu hinterfragen.

Ich denke nicht groß über mögliche Alternativen nach, wenn ich Entscheidungen treffe.

Ich wäge immer ab, was für eine Sache spricht und was dagegen.

Meine Freunde schätzen mich für mein gutes Urteilsvermögen.

Ich werde schnell ungeduldig, wenn andere mir von ihren Problemen erzählen.

Um einem Freund zu helfen, finde ich immer irgendwie Zeit.

Ich scheue keine Mühen, um Menschen aufzumuntern, die niedergeschlagen wirken.

Ich bin nur dann gut zu anderen, wenn sie auch gut zu mir sind.

Führungsvermögen [LEA]
Ich bin nicht gut darin, andere zur Zusammenarbeit zu motivieren.

Ich bin gut darin, andere zur erfolgreichen Zusammenarbeit anzuleiten.

Ich bin nicht gut darin, in einer Gruppe die Führung zu übernehmen.

Andere nehmen mich zwar als sehr bestimmende, aber gerechte Führungsperson wahr. 


\begin{tabular}{|c|c|c|c|}
\hline & & (Love of) Learning [LOV] - rev: Learning [LER] & [Liebe zum] Lernen [LOV] - rev: Wissensdrang [LER] \\
\hline 17 & - & Don't like to learn new things. (v38) & Es macht mir keinen Spaß, Neues zu lernen. \\
\hline 41 & - & Don't read nonfiction books for fun. (v332) & Sachbücher lese ich nur, wenn ich muss. \\
\hline 65 & + & Am a true life-long learner. (140) & Ich bin jemand, der wirklich sein ganzes Leben hinzulernen will. \\
\hline \multirow[t]{2}{*}{89} & + & Am thrilled when I learn something new. (v75) & Ich bin begeistert, wann immer es etwas Neues zu lernen gibt. \\
\hline & & Modesty [MOD] & Bescheidenheit [MOD] \\
\hline 11 & - & Like to stand out in a crowd. (v68) & Ich mag es, aus der Masse hervorzustechen. \\
\hline 35 & + & Don't brag about my accomplishments. (v145) & Ich prahle nie mit dem, was ich erreicht habe. \\
\hline 59 & - & Like to talk about myself. (v214) & Ich spreche gerne über mich selbst. \\
\hline \multirow[t]{2}{*}{83} & + & Would never be described as arrogant. (v321) & Niemand würde mich als arrogant bezeichnen. \\
\hline & & Originality/Creativity [ORI] & Originalität/Kreativität [ORI] \\
\hline 1 & - & Am not considered to have new and different ideas. (v189) & Ich gelte nicht gerade als einfallsreich und originell. \\
\hline 25 & + & Come up with new ways to do things. (v155) & Ich komme auf gute Ideen, wie man etwas auch ganz anders machen kann. \\
\hline 49 & + & Am an original thinker. (v226) & Ich bin ein kreativer Kopf. \\
\hline \multirow[t]{2}{*}{73} & - & Have no special urge to do something original. (v335) & Ich habe keinen besonderen Drang, etwas Originelles zu machen. \\
\hline & & Perspective [PER] & Weitsicht [PER] \\
\hline 22 & - & Am not good at figuring out what really matters. (v192) & Ich bin nicht gut darin zu erkennen, worauf es wirklich ankommt im Leben. \\
\hline 46 & + & Have a mature view on life. (v225) & Ich habe eine sehr reife Sichtweise auf das Leben. \\
\hline 70 & - & Am rarely consulted for advice by others. (v262) & Andere suchen nur selten meinen Rat. \\
\hline \multirow[t]{2}{*}{94} & + & Am considered to be a wise person. (v339) & Ich gelte als klug und weise. \\
\hline & & Prudence [PRU] & Umsicht [PRU] \\
\hline 23 & + & Believe it is always better to be safe than sorry. (v62) & Ich lebe nach dem Motto "Vorsicht ist besser als Nachsicht". \\
\hline 47 & - & Act before thinking through the consequences. (v207) & Ich handle ohne Rücksicht auf die Konsequenzen. \\
\hline 71 & - & Like taking risks. (v277) & Ich gehe gerne Risiken ein. \\
\hline \multirow[t]{2}{*}{95} & + & Make careful choices. (v311) & Bei Entscheidungen gehe ich gerne auf Nummer sicher. \\
\hline & & Self-Control [SEL] - rev: Self-Regulation [REG] & Selbstkontrolle [SEL] - rev: Selbstregulation [REG] \\
\hline 20 & + & Am a highly disciplined person. (v98) & Ich habe eine sehr gute Selbstbeherrschung. \\
\hline
\end{tabular}


$44+\quad$ Forego things that are bad for me in the long run even if they make me feel good in the short run. (v170)

68

- $\quad$ Let myself be taken over by urges to spend or eat too much. (v224)

$92 \quad$ - $\quad$ Give in to my urges. (v329)

Social/Personal Intelligence [SOC] - rev: Social Intelligence [SIQ]

14 - Don't know how to handle myself in a new social situation. (v43)

38 - Have trouble guessing how others will react. (P405)

$62+\quad$ Am good at sensing what others are feeling. (v225)

$86+\quad$ Know what to say to make people feel good. (v295)

Spirituality/Religiousness [SPI]

$\begin{array}{lll}24 & + & \text { Am a spiritual person. (v46) } \\ 48 & - & \text { Feel that life has no meaning. (Q215) } \\ 72 & + & \text { Believe that each person has a purpose in life. (v280) } \\ 96 & - & \text { Do not believe in a universal power or a God. (v282) }\end{array}$

\section{Valor/Bravery [VAL]}

$2+$ Have taken frequent stands in the face of strong opposition. (v11)

26 - Do not stand up for my beliefs. (v53)

$50+\quad$ Don't hesitate to express an unpopular opinion. (v82)

$74 \quad$ _ $\quad$ Don't speak my mind freely when there might be negative results. (v130)

\section{Zest/Vitality [ZES]}

$+\quad$ Awaken with a sense of excitement about the day's possibilities. (v326)

- Am described as grumpy. (v32)

$+\quad$ Look forward to each new day. (v182)

- Don't have much energy.
Ich verzichte auf Dinge, die mir langfristig schaden, auch wenn sie sich kurzfristig gut anfühlen.

Manchmal erliege ich der Versuchung, zu viel Geld auszugeben oder zu viel zu essen.

Meinem Verlangen gebe ich - so gut wie immer - nach.

Soziale Intelligenz [SOC] - rev: Soziale Intelligenz [SIQ]

Ich finde mich nicht gut in zwischenmenschlichen Situationen zurecht, die mir neu und unvertraut sind.

Ich bin nicht gut darin, die Reaktionen anderer Leute vorherzusehen.

Ich habe ein feines Gespür dafür, was in anderen Menschen vorgeht.

Ich weiß, was ich sagen muss, damit andere Menschen sich gut fühlen.

Ich bin ein spiritueller oder gläubiger Mensch.

Ich glaube, dass das Leben keinen tieferen Sinn hat.

Ich glaube, dass jeder Mensch eine Bestimmung im Leben hat.

Ich glaube nicht an einen Gott oder eine höhere Macht.

\section{Mut/Tapferkeit [VAL]}

Ich nehme häufig eine klare Haltung ein, auch gegen starken Widerstand.

Ich stehe nicht für meine Überzeugungen ein.

Ich schrecke nicht davor zurück, eine unbeliebte Meinung zu vertreten.

Wenn es negative Folgen haben könnte, meine Meinung zu äußern, dann halte ich sie lieber zurück.

Elan/Tatendrang [ZES]

Ich erwache mit Vorfreude auf das, was mir der Tag bringt.

Ich werde oft als mürrisch, schlecht gelaunt beschrieben.

Ich freue mich auf jeden neuen Tag.

Ich habe nur wenig Energie. 
Note. Pos. $=$ Position of item in IPIP-VIA-R questionnaire; Key = keying direction or agreement/disagreement with an item, scored 1 to $5(+)$ or 5 to $1(-)$; rev. $=$ revised scale labels (to reflect the core definition of each strength and the content of each balanced item set better) together with revised abbreviations for IPIP-VIA-R scales, which may differ from the seminal IPIP-VIA scale names and abbreviations to avoid confusion (e.g., LOV has previously referred to Love of Learning, and not Capacity for Love, which has been addressed as CAP etc.). 
Table 9

IPIP-VIA-R Reliability: Final Scales (Samples 4 and 5)

\begin{tabular}{|c|c|c|c|c|c|c|c|c|c|c|c|c|c|c|c|c|}
\hline & \multicolumn{2}{|c|}{$\mathrm{AIC}$} & \multicolumn{2}{|c|}{ AVE } & \multicolumn{2}{|c|}{ FDI } & \multicolumn{2}{|c|}{$\mathrm{gHI}$} & \multicolumn{2}{|c|}{ Alpha } & \multicolumn{2}{|c|}{ Omega } & \multicolumn{2}{|c|}{ RetestmanIFEST } & \multicolumn{2}{|c|}{ RetestLATENT } \\
\hline & UK & $\mathrm{DE}$ & UK & $\mathrm{DE}$ & UK & DE & UK & $\mathrm{DE}$ & UK & $\mathrm{DE}$ & UK & $\mathrm{DE}$ & UK & $\mathrm{DE}$ & UK & DE \\
\hline $\mathrm{APP}_{\mathrm{B}}$ & .23 & .27 & .40 & .34 & .93 & .85 & .79 & .67 & .54 & .58 & .75 & .69 & .64 & .74 & .77 & .90 \\
\hline CAP & .29 & .29 & .41 & .38 & .89 & .88 & .74 & .73 & .61 & .62 & .75 & .73 & .69 & .77 & .86 & .94 \\
\hline $\mathrm{ClT}_{\mathrm{B}}$ & .25 & .23 & .41 & .32 & .98 & .85 & .83 & .66 & .57 & .54 & .74 & .68 & .66 & .63 & .82 & .94 \\
\hline CUR & .24 & .39 & .43 & .50 & .88 & .94 & .72 & .82 & .56 & .72 & .75 & .82 & .64 & .80 & .80 & .93 \\
\hline $\mathrm{EQU}_{\mathrm{B}}$ & .30 & .33 & .47 & .42 & .91 & .91 & .76 & .77 & .63 & .67 & .80 & .78 & .58 & .71 & .73 & .83 \\
\hline $\mathrm{FOR}_{\mathrm{B}}$ & .24 & .37 & .45 & .52 & .95 & .94 & .81 & .84 & .56 & .71 & .75 & .83 & .54 & .75 & .71 & .84 \\
\hline GRA & .31 & .28 & .48 & .38 & 1.00 & .87 & .83 & .72 & .63 & .61 & .84 & .74 & .65 & .67 & .73 & .81 \\
\hline HOP & .28 & .32 & .48 & .45 & .92 & .91 & .79 & .78 & .60 & .65 & .80 & .79 & .72 & .84 & .91 & 1.00 \\
\hline HUM & .28 & .42 & .45 & .50 & .93 & .91 & .80 & .79 & .61 & .74 & .79 & .83 & .67 & .82 & .85 & .92 \\
\hline IND & .32 & .40 & .43 & .48 & .91 & .90 & .78 & .76 & .63 & .73 & .81 & .82 & .53 & .77 & .61 & .89 \\
\hline INT & .34 & .34 & .47 & .39 & .92 & .89 & .73 & .69 & .67 & .65 & .83 & .80 & .68 & .77 & .80 & .95 \\
\hline JUD & .24 & .22 & .41 & .31 & .88 & .83 & .72 & .66 & .55 & .51 & .75 & .67 & .59 & .58 & .77 & .82 \\
\hline KIN & .27 & .23 & .43 & .33 & .89 & .84 & .74 & .67 & .59 & .54 & .77 & .69 & .66 & .75 & .88 & .95 \\
\hline LEA & .25 & .34 & .45 & .40 & .91 & .90 & .77 & .77 & .57 & .67 & .76 & .76 & .64 & .75 & .85 & .94 \\
\hline $\mathrm{LOV}_{\mathrm{B}}$ & .26 & .31 & .42 & .39 & .95 & .94 & .80 & .83 & .55 & .62 & .80 & .81 & .53 & .71 & .73 & .82 \\
\hline MOD & .23 & .24 & .35 & .30 & .89 & .86 & .74 & .69 & .54 & .55 & .72 & .66 & .76 & .76 & .92 & .88 \\
\hline ORI & .29 & .38 & .45 & .47 & .90 & .90 & .78 & .77 & .62 & .71 & .79 & .80 & .72 & .79 & .96 & .95 \\
\hline PER & .25 & .28 & .44 & .41 & .87 & .86 & .71 & .70 & .56 & .60 & .74 & .74 & .73 & .70 & .94 & .88 \\
\hline $\mathrm{PRU}_{\mathrm{A}}$ & .17 & .30 & .35 & .43 & .90 & .93 & .74 & .80 & .45 & .63 & .63 & .77 & .76 & .76 & 1.00 & .90 \\
\hline $\mathrm{SEL}_{B}$ & .15 & .17 & .42 & .27 & 1.00 & .80 & .89 & .60 & .42 & .45 & .68 & .57 & .53 & .64 & .61 & .95 \\
\hline SOC & .31 & .25 & .47 & .36 & .93 & 1.00 & .80 & .87 & .63 & .56 & .81 & .72 & .69 & .66 & .84 & .80 \\
\hline $\mathrm{SPI}_{\mathrm{A}}$ & .26 & .37 & .34 & .48 & .91 & .95 & .77 & .83 & .57 & .70 & .73 & .81 & .77 & .83 & .99 & 1.00 \\
\hline VAL & .22 & .32 & .42 & .39 & .88 & .89 & .74 & .73 & .54 & .63 & .73 & .77 & .75 & .73 & .90 & .96 \\
\hline$Z_{E S}$ & .29 & .38 & .48 & .48 & 1.00 & 1.00 & .89 & .87 & .62 & .71 & .81 & .83 & .72 & .82 & .88 & .94 \\
\hline Mean & .26 & .31 & .43 & .40 & .92 & .90 & .78 & .75 & .58 & .63 & .76 & .75 & .66 & .74 & .83 & .90 \\
\hline
\end{tabular}

Note. $N=950$ (474 and 476 in UK and DE, respectively), retest- $n=224(120+124$, respectively). The presence of a scale name index indicates the item/scale variant tested (in the UK, for CIT, EQU, FOR, SEL the A-versions were used). AIC = Average Inter-item Correlation; AVE = Average Variance Extracted; FDI = Factor Determinacy Index; gHI = generalized $\mathrm{H}$-Index based on observed ordered categorical responses; Alpha = Cronbach's Alpha (internal consistency); Omega = Composite reliability for the common factor; Retest-Manifest = Test-retest reliability of strength scale score ( 3 wks. apart); Retest-Latent = Correlation of latent strength variables between test and retest ( 3 wks. apart) [estimates $>1$ were limited to 1.00 for computation of means]. The row with means represents simple mathematical averages (without Fisher's $r$-to-Z' transformation). 


\section{Table 10}

IPIP-VIA-R Measurement Invariance: UK vs Germany (Samples 4 and 5)

\begin{tabular}{|c|c|c|c|c|c|c|c|c|c|c|c|}
\hline Model $^{\mathrm{a}}$ & $X^{2}(d f)$ & $p$ & $\mathrm{CFI}$ & RMSEA & SRMR & $\mathrm{BIC}$ & $\Delta \mathrm{CFI}$ & $\triangle \mathrm{RMSEA}$ & $\triangle \mathrm{SRMR}$ & $\Delta \mathrm{BIC}$ & $\mathrm{MI}{ }^{b}, \mathrm{c}, \mathrm{d}$ \\
\hline APP - configural & $14.43(10)$ & .154 & .994 & .031 & .037 & 10977 & & & & & YES \\
\hline APP - metric & $21.50(13)$ & .064 & .988 & .037 & .051 & 10964 & -.006 & .006 & .014 & -13 & YES \\
\hline APP - scalar & $84.68(16)$ & .000 & .899 & .095 & .081 & 11010 & -.089 & .058 & .030 & 46 & NO \\
\hline APP - $\left(v_{2}\right.$ free $)$ & $22.95(15)$ & .085 & .988 & .033 & .054 & 10952 & .000 & -.004 & .003 & -12 & partial scalar \\
\hline CAP - configural & $37.92(10)$ & .000 & .959 & .077 & .050 & 11356 & & & & & YES \\
\hline CAP - metric & $43.27(13)$ & .000 & .956 & .070 & .063 & 11342 & -.003 & -.007 & .013 & -14 & YES \\
\hline CAP - scalar & $74.03(16)$ & .000 & .915 & .087 & .082 & 11352 & -.041 & .017 & .019 & 10 & NO \\
\hline CAP - $\left(v_{1}, v_{3}\right.$ free $)$ & $43.78(14)$ & .000 & .956 & .067 & .062 & 11336 & .000 & -.003 & -.001 & -6 & partial scalar \\
\hline CIT - configural & $37.12(10)$ & .000 & .957 & .076 & .080 & 10625 & & & & & YES \\
\hline $\mathrm{CIT}$ - metric & $42.12(13)$ & .000 & .954 & .069 & .092 & 10610 & -.003 & -.007 & .012 & -15 & YES \\
\hline CIT - scalar & $77.91(16)$ & .000 & .902 & .090 & .102 & 10630 & -.052 & .021 & .010 & 20 & NO \\
\hline CIT - $\left(v_{1}, v_{2}\right.$ free $)$ & $42.86(14)$ & .000 & .954 & .066 & .095 & 10603 & .000 & -.003 & .003 & -7 & partial scalar \\
\hline CUR - configural & $15.78(10)$ & .106 & .994 & .035 & .042 & 10490 & & & & & YES \\
\hline CUR - metric & $20.08(13)$ & .093 & .992 & .034 & .055 & 10474 & -.002 & -.001 & .013 & -16 & YES \\
\hline CUR - scalar & $70.52(16)$ & .000 & .939 & .085 & .066 & 10511 & -.053 & .051 & .011 & 37 & NO \\
\hline CUR - ( $v_{1}$ free) & $36.27(15)$ & .002 & .976 & .055 & .055 & 10478 & -.016 & .021 & .000 & 4 & ((partial scalar)) \\
\hline EQU - configural & $15.02(10)$ & .131 & .993 & .033 & .042 & 10401 & & & & & YES \\
\hline EQU - metric & $31.21(13)$ & .003 & .974 & .054 & .070 & 10400 & -.019 & .021 & .028 & -1 & (YES) \\
\hline EQU - scalar & $61.35(16)$ & .000 & .935 & .077 & .088 & 10416 & -.039 & .023 & .018 & 16 & NO \\
\hline EQU - ( $v_{1}$ free $)$ & $37.50(15)$ & .001 & .986 & .056 & .073 & 10393 & .012 & .002 & .003 & -7 & partial scalar \\
\hline FOR - config & $28.41(10)$ & .002 & .978 & .062 & .058 & 10567 & & & & & YES \\
\hline FOR - metric & $38.28(13)$ & .000 & .970 & .064 & .071 & 10557 & -.008 & .002 & .013 & -10 & YES \\
\hline FOR - scalar & $43.84(16)$ & .000 & .967 & .061 & .072 & 10542 & -.003 & -.003 & .001 & -15 & scalar \\
\hline
\end{tabular}




\begin{tabular}{|c|c|c|c|c|c|c|c|c|c|c|c|}
\hline Model $^{\mathrm{a}}$ & $\mathrm{X}^{2}(d f)$ & $p$ & $\mathrm{CFI}$ & RMSEA & SRMR & $\mathrm{BIC}$ & $\Delta \mathrm{CFI}$ & $\triangle \mathrm{RMSEA}$ & $\triangle \mathrm{SRMR}$ & $\Delta \mathrm{BIC}$ & $\mathrm{MI}^{\mathrm{b}, \mathrm{c}, \mathrm{d}}$ \\
\hline GRA - configural & $34.29(10)$ & .000 & .969 & .072 & .074 & 10429 & & & & & YES \\
\hline GRA - metric & $63.05(13)$ & .000 & .936 & .090 & .117 & 10441 & -.033 & .018 & .043 & 12 & NO \\
\hline GRA - scalar & $104.7(16)$ & .000 & .887 & .108 & .130 & 10465 & -.049 & .018 & .013 & 24 & NO \\
\hline GRA - ( $\lambda_{1}$ free $)$ & $42.09(12)$ & .000 & .962 & .073 & .083 & 10424 & -.007 & .001 & .009 & -5 & partial metric \\
\hline GRA - $\left(\lambda_{1}, v_{2}\right.$ free $)$ & $45.20(14)$ & .000 & .960 & .068 & .084 & 10413 & -.002 & -.005 & .001 & -17 & partial scalar \\
\hline HOP*- configural & 24.82 (8) & .002 & .983 & .067 & .046 & 10778 & & & & & YES \\
\hline HOP*-metric & $33.58(11)$ & .000 & .977 & .066 & .057 & 10767 & -.006 & -.001 & .011 & -11 & YES \\
\hline HOP*-scalar & 93.15 (14) & .000 & .919 & .109 & .081 & 10804 & -.058 & .043 & .024 & 37 & NO \\
\hline HOP*- ( $v_{1}$ free $)$ & $34.58(13)$ & .001 & .978 & .059 & .059 & 10754 & .001 & -.007 & .002 & -13 & partial scalar \\
\hline HUM - configural & $15.66(10)$ & .110 & .994 & .035 & .031 & 10821 & & & & & YES \\
\hline HUM - metric & $27.02(13)$ & .012 & .986 & .048 & .051 & 10812 & -.008 & .013 & .020 & -9 & YES \\
\hline HUM - scalar & $51.79(16)$ & .000 & .963 & .069 & .062 & 10817 & -.023 & .021 & .011 & 5 & $(($ scalar $))$ \\
\hline IND - configural & $22.04(10)$ & .015 & .984 & .050 & .044 & 10341 & & & & & YES \\
\hline IND - metric & $33.55(13)$ & .001 & .973 & .058 & .063 & 10334 & -.011 & .008 & .019 & -7 & YES \\
\hline IND - scalar & $48.10(16)$ & .000 & .958 & .065 & .072 & 10332 & -.015 & .007 & .009 & -2 & scalar \\
\hline INT - configural & $23.74(10)$ & .008 & .979 & .054 & .081 & 9665 & & & & & YES \\
\hline INT - metric & $25.06(13)$ & .023 & .981 & .044 & .085 & 9645 & .002 & -.010 & .004 & -20 & YES \\
\hline INT - scalar & $43.64(16)$ & .000 & .957 & .060 & .092 & 9650 & -.024 & .016 & .007 & 5 & $(($ scalar $))$ \\
\hline JUD - configural & $23.84(10)$ & .008 & .980 & .054 & .051 & 10506 & & & & & YES \\
\hline JUD - metric & $23.46(13)$ & .036 & .985 & .041 & .052 & 10486 & .005 & -.013 & .001 & -20 & YES \\
\hline JUD - scalar & $29.46(16)$ & .021 & .981 & .042 & .051 & 10472 & -.004 & .001 & -.001 & -14 & scalar \\
\hline KIN - configural & $29.34(10)$ & .001 & .973 & .064 & .062 & 10700 & & & & & YES \\
\hline $\mathrm{KIN}$ - metric & 33.65 (13) & .001 & .971 & .058 & .071 & 10684 & -.002 & -.006 & .009 & -16 & YES \\
\hline KIN - scalar & $68.91(16)$ & .000 & .926 & .083 & .085 & 10700 & -.045 & .025 & .014 & 16 & NO \\
\hline KIN - $\left(v_{1}, v_{2}\right.$ free $)$ & $35.42(14)$ & .001 & .970 & .057 & .077 & 10678 & -.001 & -.001 & .006 & -6 & partial scalar \\
\hline
\end{tabular}

(continues) 


\begin{tabular}{|c|c|c|c|c|c|c|c|c|c|c|c|}
\hline Model $^{\mathrm{a}}$ & $\mathrm{X}^{2}(d f)$ & $p$ & $\mathrm{CFI}$ & RMSEA & SRMR & $\mathrm{BIC}$ & $\Delta \mathrm{CFI}$ & $\triangle \mathrm{RMSEA}$ & $\triangle \mathrm{SRMR}$ & $\Delta \mathrm{BIC}$ & $\mathrm{Ml}{ }^{\mathrm{b}}, \mathrm{c}, \mathrm{d}$ \\
\hline LEA*- configural & 14.82 (8) & .063 & .992 & .042 & .037 & 10949 & & & & & YES \\
\hline LEA*- metric & $19.66(11)$ & .050 & .990 & .041 & .040 & 10934 & -.002 & -.001 & .003 & -15 & YES \\
\hline LEA*-scalar & $32.26(14)$ & .004 & .978 & .052 & .046 & 10927 & -.012 & .011 & .006 & -7 & scalar \\
\hline LOV - configural & $19.06(10)$ & .040 & .991 & .044 & .041 & 10980 & & & & & YES \\
\hline LOV - metric & $40.73(13)$ & .000 & .971 & .067 & .044 & 10982 & -.020 & .023 & .003 & 2 & (metric) \\
\hline LOV - scalar & $95.17(16)$ & .000 & .918 & .102 & .053 & 11016 & -.053 & .035 & .009 & 34 & NO \\
\hline LOV - $\left(v_{4}, v_{2}\right.$ free $)$ & $44.07(14)$ & .000 & .969 & .067 & .046 & 10978 & -.002 & .000 & .002 & -4 & partial scalar \\
\hline MOD - configural & $40.45(10)$ & .000 & .944 & .080 & .050 & 11625 & & & & & YES \\
\hline MOD - metric & $46.24(13)$ & .000 & .938 & .073 & .058 & 11611 & -.006 & -.007 & .008 & -14 & YES \\
\hline MOD - scalar & $56.15(16)$ & .000 & .926 & .073 & .061 & 11599 & -.012 & .000 & .003 & -12 & scalar \\
\hline ORI - configural & $33.27(10)$ & .000 & .975 & .070 & .044 & 10695 & & & & & YES \\
\hline ORI - metric & $41.76(13)$ & .000 & .969 & .068 & .062 & 10685 & -.006 & -.002 & .018 & -10 & YES \\
\hline ORI - scalar & $104.87(16)$ & .000 & .903 & .108 & .092 & 10728 & -.066 & .040 & .030 & 43 & NO \\
\hline ORI - $\left(v_{2}, v_{4}\right.$ free $)$ & $41.66(14)$ & .000 & .970 & .064 & .062 & 10678 & .001 & -.004 & .000 & -7 & partial scalar \\
\hline PER - configural & $13.09(10)$ & .218 & .996 & .026 & .039 & 10237 & & & & & YES \\
\hline PER - metric & $19.21(13)$ & .117 & .993 & .032 & .039 & 10224 & -.003 & .006 & .000 & -13 & YES \\
\hline PER - scalar & $35.57(16)$ & .003 & .978 & .051 & .039 & 10221 & -.015 & .019 & .000 & -3 & (scalar) \\
\hline PRU - configural & $32.37(10)$ & .000 & .970 & .069 & .056 & 10861 & & & & & YES \\
\hline PRU - metric & $33.52(13)$ & .001 & .972 & .058 & .056 & 10842 & .002 & -.011 & .000 & -19 & YES \\
\hline PRU - scalar & $94.39(16)$ & .000 & .893 & .102 & .081 & 10885 & -.079 & .044 & .025 & 43 & NO \\
\hline PRU - ( $v_{2}$ free $)$ & $41.02(15)$ & .000 & .965 & .060 & .059 & 10837 & -.007 & .002 & .003 & -5 & partial scalar \\
\hline SEL - configural & $21.86(10)$ & .016 & .982 & .050 & .042 & 11066 & & & & & YES \\
\hline SEL - metric & $34.15(13)$ & .001 & .968 & .059 & .047 & 11059 & -.014 & .009 & .005 & -7 & YES \\
\hline SEL - scalar & $102.8(16)$ & .000 & .869 & .107 & .070 & 11097 & -.099 & .048 & .023 & 38 & NO \\
\hline$S E L-\left(v_{3}, v_{2}\right.$ free $)$ & $37.76(14)$ & .001 & .964 & .060 & .052 & 11056 & -.004 & .001 & .005 & -3 & partial scalar \\
\hline
\end{tabular}

(continues) 


\begin{tabular}{|c|c|c|c|c|c|c|c|c|c|c|c|}
\hline Model $^{\mathrm{a}}$ & $X^{2}(d f)$ & $p$ & $\mathrm{CFI}$ & RMSEA & SRMR & $\mathrm{BIC}$ & $\Delta \mathrm{CFI}$ & $\triangle \mathrm{RMSEA}$ & $\triangle \mathrm{SRMR}$ & $\Delta \mathrm{BIC}$ & $\mathrm{MI}{ }^{\mathrm{b}}, \mathrm{c}, \mathrm{d}$ \\
\hline SOC - configural & $22.35(10)$ & .013 & .985 & .051 & .040 & 10497 & & & & & YES \\
\hline SOC - metric & $33.02(13)$ & .002 & .976 & .057 & .045 & 10488 & -.009 & .006 & .005 & -9 & YES \\
\hline SOC - scalar & $35.58(16)$ & .003 & .977 & .051 & .048 & 10470 & .001 & -.006 & .003 & -18 & scalar \\
\hline SPI*- configural & 12.42 & .134 & .995 & .034 & .032 & 12245 & & & & & YES \\
\hline SPI*-metric & $19.26(11)$ & .057 & .990 & .040 & .039 & 12231 & -.005 & .006 & .007 & -14 & YES \\
\hline SPI*-scalar & $37.58(14)$ & .001 & .971 & .060 & .048 & 12229 & -.019 & .020 & .009 & -2 & (scalar) \\
\hline VAL - configural & $24.16(10)$ & .007 & .980 & .055 & .055 & 10729 & & & & & YES \\
\hline VAL - metric & $29.75(13)$ & .005 & .977 & .052 & .048 & 10715 & -.003 & -.003 & -.007 & -14 & YES \\
\hline VAL - scalar & $67.47(16)$ & .000 & .928 & .082 & .081 & 10733 & -.049 & .030 & .033 & 18 & NO \\
\hline VAL - ( $v_{1}$ free $)$ & $38.96(15)$ & .001 & .967 & .058 & .064 & 10710 & -.010 & .006 & .015 & -5 & partial scalar \\
\hline ZES - configural & $27.63(10)$ & .002 & .982 & .061 & .053 & 10735 & & & & & YES \\
\hline ZES - metric & $31.34(13)$ & .003 & .982 & .055 & .055 & 10718 & .000 & -.006 & .002 & -17 & YES \\
\hline ZES - scalar & $36.17(16)$ & .003 & .980 & .052 & .051 & 10702 & -.002 & -.003 & -.004 & -16 & scalar \\
\hline
\end{tabular}

Note. ${ }^{a}$ Greek letters reflect freed parameters in partial invariance models (e.g., $\lambda_{1} / \mathrm{V}_{2}$ refer to free loading/intercept, respectively, of the indexed items \#1 and \#2); models with asterisk contain one pair of correlated residuals: HOP*, LEA*, SPI*; ${ }^{b} \mathrm{MI}=$ Measurement invariance level attainment: Configural invariance achieved for all scales; ${ }^{\mathrm{c}} \mathrm{Metric}$ invariance achieved for all scales except: GRA (EQU \& LOV permissible according to BIC, but set in parentheses), partial metric invariance achieved for GRA; d Scalar invariance achieved for eleven scales: FOR, JUD, LEA*, MOD, IND, PER, SOC, SPI*, ZES (HUM \& INT tentatively permissible according to BIC, but set in double parentheses), partial scalar invariance achieved for APP, CAP, CIT, EQU, GRA, HOP, KIN, LOV, ORI, PRU, SEL, VAL (CUR tentatively permissible according to BIC, but set in double parentheses). Delta-fit values for partial invariance models are derived against the last accepted (full or partial) invariance model. 
Table 11

Associations of Character Strength Scales: IPIP-VIA-R Inter-Correlations (Samples 4 and 5)

\begin{tabular}{|c|c|c|c|c|c|c|c|c|c|c|c|c|c|c|c|c|c|c|c|c|c|c|c|c|}
\hline & APP & CAP & $\mathrm{CIT}$ & CUR $^{a}$ & EQU & FOR & GRA & HOP & HUM & IND & INT & JUD & KIN & LEA & LOV & MOD & ORI & PER & PRU & SEL & SOC & SPI & VAL & $\mathrm{ZES}^{\mathrm{a}}$ \\
\hline$\overline{\text { APP }}$ & - & .45 & .34 & .49 & .41 & .22 & .52 & .34 & .39 & .34 & .43 & .38 & .40 & .29 & .46 & .15 & .42 & .45 & .22 & .30 & .41 & .34 & .24 & .41 \\
\hline CAP & .42 & - & .49 & .42 & .35 & .34 & .53 & .39 & .49 & .35 & .31 & .30 & .42 & .30 & .32 & .11 & .39 & .40 & .12 & .18 & .45 & .24 & .26 & .55 \\
\hline CIT & .42 & .57 & - & .46 & .39 & .34 & .45 & .31 & .45 & .41 & .41 & .32 & .52 & .40 & .32 & .13 & .32 & .42 & .13 & .18 & .39 & .17 & .31 & .46 \\
\hline CUR $^{a}$ & .53 & .49 & .57 & - & .32 & .31 & .47 & .45 & .51 & .41 & .34 & .32 & .51 & .45 & .62 & -.10 & .57 & .52 & -.02 & .22 & .44 & .28 & .31 & .59 \\
\hline EQU & .44 & .34 & .47 & .46 & - & .36 & .48 & .34 & .23 & .39 & .50 & .28 & .48 & .17 & .30 & .32 & .17 & .30 & .26 & .33 & .28 & .24 & .15 & .37 \\
\hline FOR & .23 & .36 & .42 & .37 & .50 & - & .39 & .43 & .23 & .13 & .17 & .02 & .41 & .17 & .24 & .04 & .13 & .21 & -.03 & .17 & .20 & .13 & .07 & .44 \\
\hline GRA & .57 & .56 & .60 & .53 & .58 & .41 & - & .39 & .44 & .40 & .48 & .35 & .54 & .24 & .40 & .14 & .32 & .48 & .20 & .23 & .41 & .36 & .24 & .50 \\
\hline HOP & .27 & .39 & .44 & .47 & .33 & .44 & .38 & - & .43 & .41 & .26 & .20 & .38 & .40 & .39 & .03 & .37 & .47 & -.14 & .34 & .41 & .24 & .28 & .61 \\
\hline HUM & .40 & .46 & .56 & .55 & .42 & .32 & .50 & .44 & - & .38 & .28 & .33 & .46 & .50 & .43 & -.05 & .57 & .51 & -.14 & .12 & .52 & .19 & .42 & .57 \\
\hline IND & .32 & .39 & .48 & .48 & .42 & .32 & .47 & .43 & .41 & - & .50 & .43 & .38 & .38 & .41 & .18 & .35 & .51 & .20 & .37 & .35 & .11 & .44 & .49 \\
\hline INT & .46 & .33 & .52 & .41 & .54 & .30 & .60 & .29 & .40 & .55 & - & .42 & .47 & .18 & .30 & .35 & .23 & .40 & .31 & .35 & .27 & .16 & .35 & .31 \\
\hline JUD & .48 & .33 & .45 & .50 & .45 & .31 & .51 & .31 & .41 & .48 & .51 & - & .34 & .34 & .37 & .08 & .37 & .50 & .30 & .19 & .48 & .16 & .34 & .24 \\
\hline KIN & .45 & .47 & .61 & .54 & .60 & .46 & .65 & .42 & .48 & .48 & .58 & .48 & - & .38 & .40 & .22 & .36 & .42 & .10 & .23 & .45 & .27 & .33 & .46 \\
\hline LEA & .31 & .43 & .56 & .48 & .23 & .28 & .38 & .45 & .50 & .45 & .33 & .41 & .40 & - & .44 & -.19 & .51 & .49 & -.16 & .16 & .45 & .20 & .39 & .41 \\
\hline LOV & .48 & .33 & .46 & .58 & .50 & .34 & .48 & .25 & .42 & .39 & .40 & .52 & .44 & .35 & - & -.04 & .46 & .44 & .02 & .17 & .38 & .20 & .28 & .44 \\
\hline MOD & .25 & .09 & .19 & .04 & .27 & .06 & .34 & .02 & .14 & .23 & .41 & .25 & .28 & .03 & .12 & - & -.16 & -.03 & .34 & .18 & -.08 & .02 & -.04 & .05 \\
\hline ORI & .40 & .36 & .43 & .61 & .33 & .29 & .35 & .37 & .43 & .41 & .33 & .48 & .37 & .51 & .51 & -.12 & - & .54 & -.14 & .15 & .49 & .22 & .37 & .38 \\
\hline PER & .44 & .46 & .49 & .56 & .41 & .30 & .58 & .43 & .48 & .51 & .51 & .62 & .53 & .55 & .45 & .17 & .49 & - & .07 & .32 & .55 & .25 & .37 & .49 \\
\hline PRU & .28 & .10 & .14 & .07 & .26 & .04 & .24 & -.09 & .07 & .20 & .35 & .37 & .19 & .01 & .12 & .34 & -.03 & .24 & - & .19 & .04 & .06 & -.07 & -.04 \\
\hline SEL & .20 & .13 & .21 & .26 & .24 & .31 & .23 & .32 & .16 & .37 & .40 & .34 & .24 & .23 & .17 & .21 & .23 & .34 & .31 & - & .24 & .15 & .12 & .28 \\
\hline SOC & .44 & .55 & .57 & .57 & .40 & .37 & .52 & .51 & .57 & .49 & .39 & .57 & .51 & .64 & .41 & .07 & .51 & .61 & .11 & .28 & - & .27 & .32 & .42 \\
\hline SPI & .25 & .35 & .36 & .35 & .28 & .38 & .33 & .36 & .28 & .31 & .26 & .19 & .30 & .27 & .27 & .01 & .28 & .27 & .06 & .22 & .39 & - & .05 & .29 \\
\hline VAL & .26 & .30 & .31 & .39 & .27 & .19 & .33 & .32 & .38 & .36 & .27 & .38 & .27 & .45 & .35 & -.10 & .51 & .47 & -.04 & .10 & .41 & .19 & - & .24 \\
\hline $\mathrm{ZES}^{\mathrm{a}}$ & .28 & .45 & .47 & .56 & .35 & .41 & .39 & .61 & .46 & .48 & .33 & .32 & .41 & .43 & .32 & -.03 & .40 & .39 & .02 & .37 & .51 & .42 & .27 & - \\
\hline
\end{tabular}

Note: $N=950$ (474 in UK; 476 in DE); ${ }^{a} N=475$ (in DE); UK = below diagonal; DE = above diagonal. For UK and DE, $p<.05$ if $|r|>.09 ; p<.01$ if $|r|>.12, p<.001$ if $|r|>.16$. 


\section{Table 12}

IPIP-VIA-R Nomological Net: Cross-Country Comparison of Correlations of Character Strengths with Big Five Domains and Human Value Clusters (Samples 4 and 5)

\begin{tabular}{|c|c|c|c|c|c|c|c|c|c|c|c|c|c|c|c|c|c|c|}
\hline \multirow[b]{2}{*}{ Scale } & \multicolumn{2}{|c|}{$\mathrm{E}$} & \multicolumn{2}{|c|}{$A$} & \multicolumn{2}{|c|}{$C$} & \multicolumn{2}{|r|}{$\mathrm{N}$} & \multicolumn{2}{|r|}{0} & \multicolumn{2}{|c|}{ S-T } & \multicolumn{2}{|c|}{ S-E } & \multicolumn{2}{|c|}{$\mathrm{O}-\mathrm{C}$} & \multicolumn{2}{|c|}{ CON } \\
\hline & UK & $\mathrm{DE}$ & UK & DE & UK & $\mathrm{DE}$ & UK & $\mathrm{DE}$ & UK & DE & UK & DE & UK & $\mathrm{DE}$ & UK & DE & UK & $\mathrm{DE}$ \\
\hline APP & .14 & .25 & .40 & .41 & .31 & .35 & -.12 & -.29 & .51 & .48 & .38 & .34 & -.31 & -.26 & .03 & .00 & -.06 & -.08 \\
\hline CAP & .36 & .35 & .47 &. .45 & .35 & .35 & -.31 & -.38 & .31 & .29 & .25 & .24 & -.22 & -.23 & .17 & .08 & -.18 & -.10 \\
\hline $\mathrm{CIT}$ & .38 & .36 & .54 & .45 & .45 & .33 & -.31 & -.37 & .37 & .25 & .35 & .29 & -.27 & -.16 & .08 & .01 & -.12 & -.12 \\
\hline CUR & .43 & $.50^{\mathrm{a}}$ & .42 & $.41^{\mathrm{a}}$ & .36 & $.33^{a}$ & -.38 & $-.45^{\mathrm{a}}$ & .52 & $.57^{\mathrm{a}}$ & .23 & $.22^{\mathrm{c}}$ & -.24 & $-.09^{b}$ & .32 & $.20^{c}$ & -.27 & $-.31^{d}$ \\
\hline EQU & .12 & .12 & .63 & .61 & .35 & .41 & -.19 & -.31 & .41 & .24 & .60 & .50 & -.44 & -.39 & -.03 & -.14 & -.11 & .06 \\
\hline FOR & .23 & .21 & .57 &.$\underline{.42}$ & .34 & .13 & -.34 & -.41 & .34 & .15 & .38 & .23 & -.29 & -.14 & .05 & .03 & -.11 & -.11 \\
\hline GRA & .17 & .24 & .61 & .54 & .42 & .37 & -.24 & -.33 & .36 & .32 & .48 & .35 & -.43 & -.28 & .00 & -.04 & -.02 & -.03 \\
\hline HOP & .46 & .43 & .44 & .38 & .37 & .32 & -.58 & -.73 & .30 & .39 & .17 & .17 & -.24 & -.16 & .27 & .19 & -.17 & -.19 \\
\hline HUM & .40 & .58 & .43 & .38 & .34 & .25 & -.34 & -.46 & .35 & .45 & .25 & .10 & -.27 & -.09 & .27 & .31 & -.21 & -.32 \\
\hline IND & .27 & .38 & .45 & .32 & .59 & .69 & -.37 & -.53 & .31 & .32 & .21 & .13 & -.28 & -.13 & .07 & .00 & -.01 & -.01 \\
\hline INT & .07 & .16 & .56 & .48 & .54 & .55 & -.25 & -.28 & .28 & .21 & .40 & .37 & -.46 & -.39 & -.09 & -.07 & .12 & .08 \\
\hline JUD & .14 & .19 & .43 & .26 & .48 & .37 & -.26 & -.23 & .43 & .34 & .29 & .24 & -.32 & -.13 & .00 & -.13 & .02 & .02 \\
\hline KIN & .20 & .32 & .64 & .61 & .43 & .38 & -.24 & -.39 & .36 & .35 & .50 & .46 & -.46 & -.38 & .06 & .03 & -.08 & -.09 \\
\hline LEA & .60 & .66 & .30 & .22 & .39 & .31 & -.42 & -.45 & .34 & .46 & .07 & .03 & -.08 & .08 & .23 & .21 & -.18 & -.33 \\
\hline LOV & .19 & .41 & .39 & .34 & .31 & .27 & -.10 & -.35 & .52 & .56 & .36 & .21 & -.25 & -.02 & .12 & .11 & -.20 & -.28 \\
\hline MOD & -.30 & -.30 & .36 & .30 & .29 & .25 & .00 & -.07 & -.02 & -.22 & .37 & .31 & -.44 & -.52 & -.36 & -.24 & .38 & .43 \\
\hline ORI & .42 & .52 & .27 & .24 & .28 & .24 & -.27 & -.32 & .62 & .67 & .11 & .06 & -.06 & .00 & .38 & .33 & -.38 & -.37 \\
\hline PER & .33 & .46 & .43 & .38 & .48 & .45 & -.41 & -.52 & .39 & .52 & .26 & .20 & -.31 & -.07 & .11 & .07 & -.04 & -.18 \\
\hline
\end{tabular}




\begin{tabular}{|c|c|c|c|c|c|c|c|c|c|c|c|c|c|c|c|c|c|c|}
\hline \multirow[b]{2}{*}{ Scale } & \multicolumn{2}{|c|}{$\mathrm{E}$} & \multicolumn{2}{|c|}{ A } & \multicolumn{2}{|c|}{ C } & \multicolumn{2}{|c|}{$\mathrm{N}$} & \multicolumn{2}{|r|}{0} & \multicolumn{2}{|c|}{ S-T } & \multicolumn{2}{|c|}{ S-E } & \multicolumn{2}{|c|}{$\mathrm{O}-\mathrm{C}$} & \multicolumn{2}{|c|}{ CON } \\
\hline & UK & $\mathrm{DE}$ & UK & $\mathrm{DE}$ & UK & $\mathrm{DE}$ & UK & $\mathrm{DE}$ & UK & $\mathrm{DE}$ & UK & $\mathrm{DE}$ & UK & $\mathrm{DE}$ & UK & DE & UK & $\mathrm{DE}$ \\
\hline PRU & -.25 & -.30 & .23 & .21 & .33 & .33 & .02 & .11 & .05 & -.12 & .17 & .21 & -.26 & -.20 & -.42 & -.52 & .45 & .51 \\
\hline SEL & .17 & . 15 & .34 & .27 & .52 & .43 & -.43 & -.38 & .17 & .17 & .16 & .20 & -.30 & -.26 & -.06 & -.07 & .18 & .12 \\
\hline SOC & .48 & .43 & .45 & .39 & .47 & .32 & -.39 & -.36 & .40 & .49 & .21 & .25 & -.24 & -.11 & .20 & .13 & -.14 & -.25 \\
\hline SPI & .25 & .21 & .36 & .29 & .21 & .19 & -.25 & -.17 & .25 & .29 & .20 & .20 & -.26 & -.16 & .04 & -.11 & .05 & .06 \\
\hline VAL & .37 & .41 & .15 & .11 & .20 & .27 & -.24 & -.28 & .36 & .32 & .18 & .14 & -.13 & -.12 & .28 & .23 & -.29 & -.26 \\
\hline ZES & .56 & $.54^{\mathrm{a}}$ & .47 & $.48^{\mathrm{a}}$ & .47 & $.44^{\mathrm{a}}$ & -.58 & $-.69^{\mathrm{a}}$ & .33 & $.37^{\mathrm{a}}$ & .11 & $.17^{c}$ & -.19 & $-.18^{b}$ & .30 & $.18^{\mathrm{c}}$ & -.19 & $-.17^{\mathrm{d}}$ \\
\hline$N$ & 474 & 476 & 474 & 476 & 474 & 476 & 474 & 476 & 474 & 476 & 438 & 440 & 444 & 448 & 442 & 440 & 423 & 431 \\
\hline
\end{tabular}

Note. ${ }^{\mathrm{a}} \mathrm{N}=475,{ }^{\mathrm{b}} \mathrm{N}=447,{ }^{\mathrm{c}} \mathrm{N}=439,{ }^{\mathrm{d}} \mathrm{N}=430 ; \mathrm{UK}=$ United Kingdom, DE = Germany; Scale = IPIP-VIA-R strength; Big Five Domains: E = Extraversion, A = Agreeableness, C = Conscientiousness, $\mathrm{N}=$ Neg. Emotionality (Neuroticism), $\mathrm{O}=$ Open-Mindedness; Schwartz Value Higher-Order Dimensions (centered): S-T = Self-Transcendence, S-E= Self-Enhancement, $\mathrm{O}-\mathrm{C}=$ Openness-to-Change, $\mathrm{CON}=$ Conservation, with Schwartz value cluster means based on ipsatively centered item scores. For all coefficients, $p<.05$ if $|r|>.09 ; p<.01$ if $|r|$ $>.12, p<.001$ if $|r|>.16$, except $r_{\text {PRU }} \sim$ ODE and $r_{\text {PRU }}$ - - T|UK $(p<.01)$. Underlining refers to the hypothesized strongest (absolute) correlation per country with respect to two frameworks (Big Five and Schwartz human values); bold type font reflects the empirically highest coefficients per country within each framework; underlined bold font indicates a confirmed hypothesis (accuracy of comparisons: only to the level of digits as shown). 
Table 13a

IPIP-VIA-R Construct Validity: Multiple Regression on Big Five Domains and Facets (Sample 4: UK)

\begin{tabular}{|c|c|c|c|c|c|c|c|c|c|c|c|}
\hline \multicolumn{6}{|c|}{ Big Five Domains } & \multicolumn{6}{|c|}{ Big Five Facets } \\
\hline Scale & Domain & $r$ & $r^{+}$ & $r^{\ddagger}$ & $R_{\text {adj }}$ & Scale & Facet & $r$ & $r^{\dagger}$ & $r^{\ddagger}$ & $R_{\text {adj }}$ \\
\hline KIN & $A$ & .64 & .75 & .91 & .67 & ORI & O-c & .66 & .87 & $1.04 \bullet$ & .71 \\
\hline EQU & $A$ & .63 & .74 & .88 & .67 & IND & $C-p$ & .64 & .87 & $1.04 \bullet$ & .68 \\
\hline ORI & $\mathrm{O}$ & .62 & .73 & .87 & .67 & ZES & $N-d$ & .61 & .74 & .88 & .77 \\
\hline GRA & $A$ & .61 & .72 & .84 & .64 & INT & $C-r$ & .60 & $1.02 \bullet$ & $1.19 \bullet$ & .67 \\
\hline LEA & $\mathrm{E}$ & .60 & .72 & .87 & .66 & GRA & $A-C$ & .58 & .87 & $1.02 \bullet$ & .68 \\
\hline IND & C & .59 & .68 & .81 & .63 & EQU & $A-r$ & .56 & .83 & .97 & .67 \\
\hline ZES & $\mathrm{N}$ & .58 & .63 & .75 & .73 & LEA & E-a & .56 & .75 & .90 & .71 \\
\hline HOP & $\mathrm{N}$ & .58 & .62 & .74 & .67 & KIN & $A-C$ & .55 & .83 & $1.00 \bullet$ & .68 \\
\hline FOR & $A$ & .57 & .67 & .81 & .61 & HOP & $N-d$ & .55 & .67 & .79 & .69 \\
\hline INT & $A$ & .56 & .65 & .76 & .64 & FOR & $A-t$ & .54 & .78 & .95 & .62 \\
\hline CIT & $A$ & .54 & .64 & .79 & .65 & JUD & C-r & .50 & .85 & $1.05 \bullet$ & .60 \\
\hline CUR & 0 & .52 & .62 & .75 & .65 & CUR & O-c & .49 & .64 & .79 & .67 \\
\hline LOV & 0 & .52 & .61 & .75 & .58 & CIT & $A-C$ & .48 & .72 & .89 & .67 \\
\hline SEL & $C$ & .52 & .59 & .76 & .56 & PER & $C-r$ & .48 & .81 & .98 & .64 \\
\hline APP & 0 & .51 & .60 & .75 & .57 & CAP & $A-C$ & .47 & .71 & .86 & .64 \\
\hline SOC & $\mathrm{E}$ & .48 & .58 & .68 & .66 & SOC & C-r & .45 & .77 & .91 & .69 \\
\hline JUD & C & .48 & .55 & .68 & .59 & APP & $0-i$ & .45 & .72 & .90 & .59 \\
\hline PER & $C$ & .48 & .55 & .66 & .60 & LOV & $0-i$ & .44 & .71 & .87 & .59 \\
\hline CAP & $A$ & .47 & .55 & .67 & .56 & MOD & A-r & .43 & .64 & .82 & .57 \\
\hline HUM & $A$ & .43 & .50 & .59 & .56 & SEL & $\mathrm{N}-\mathrm{e}$ & .43 & .49 & .64 & .59 \\
\hline VAL & $E$ & .37 & .45 & .56 & .45 & VAL & E-a & .41 & .54 & .67 & .54 \\
\hline SPI & A & .36 & .43 & .55 & .42 & HUM & O-c & .40 & .53 & .63 & .59 \\
\hline MOD & $A$ & .36 & .42 & .54 & .54 & PRU & C-r & .37 & .63 & .84 & .54 \\
\hline PRU & C & .33 & .38 & .51 & .49 & SPI & $A-C$ & .32 & .49 & .62 & .45 \\
\hline Mean & & .51 & .60 & .73 & .60 & Mean & & .50 & .73 & .87 & .64 \\
\hline
\end{tabular}

Notes. $N=474$. Scale = IPIP-VIA-R strength; $r=$ max. (uncorrected) bivariate absolute correlation with BFI-2-S domain or facet (in descending order); $r^{\dagger}=$ single disattenuation for unreliability in BFI-2-S domain (Cronbach's Alpha for six items) or BFI-2-S facet (Spearman-Brown-correction for two-item correlation; see Eisinga et al., 2013); $r^{\ddagger}=$ double disattenuation for unreliability in BFI-2-S and strength scale (Omega estimate). $R_{\text {adj }}=$ multiple-R (square root of $R^{2}$ adj) for full models regressing strength on five BFI-2-S domains or 15 BFI-2-S facets); • overadjustment (estimates $>1$ were limited to 1.00 for computation of means). 
Table 13b

IPIP-VIA-R Construct Validity: Multiple-Regression on Big Five Domains and Facets (Sample 5: DE)

\begin{tabular}{|c|c|c|c|c|c|c|c|c|c|c|c|}
\hline \multicolumn{6}{|c|}{ Big Five Domains } & \multicolumn{6}{|c|}{ Big Five Facets } \\
\hline Scale & Domain & $r$ & $r^{\dagger}$ & $r^{\ddagger}$ & $R_{\text {adj }}$ & Scale & Facet & $r$ & $r^{\dagger}$ & $r^{\ddagger}$ & $R_{\mathrm{ad}}$ \\
\hline HOP & $\mathrm{N}$ & .73 & .80 & .94 & .75 & ORI & O-c & .75 & .91 & $1.05 \bullet$ & .77 \\
\hline ZES $^{a}$ & $\mathrm{~N}$ & .69 & .76 & .88 & .76 & IND & $C-p$ & .70 & .93 & $1.06 \bullet$ & .77 \\
\hline IND & $\mathrm{C}$ & .69 & .77 & .89 & .74 & ZES $^{a}$ & $N-d$ & .69 & .82 & .94 & .80 \\
\hline ORI & 0 & .67 & .78 & .90 & .70 & HOP & $\mathrm{N}-\mathrm{a}$ & .67 & .94 & $1.10 \bullet$ & .76 \\
\hline LEA & $E$ & .66 & .78 & .93 & .69 & LEA & $E-a$ & .63 & .77 & .92 & .71 \\
\hline EQU & $A$ & .61 & .73 & .87 & .64 & INT & C-r & .61 & .87 & $1.05 \bullet$ & .65 \\
\hline KIN & $A$ & .61 & .72 & .93 & .65 & $\mathrm{KIN}$ & A-c & .60 & .92 & $1.18 \bullet$ & .67 \\
\hline HUM & $\mathrm{E}$ & .58 & .68 & .78 & .65 & EQU & A-r & .57 & .80 & .96 & .65 \\
\hline CUR $^{a}$ & 0 & .57 & .66 & .76 & .67 & CUR $^{a}$ & E-e & .56 & .74 & .84 & .69 \\
\hline LOV & 0 & .56 & .66 & .80 & .61 & PER & O-c & .54 & .66 & .80 & .69 \\
\hline INT & $C$ & .55 & .62 & .74 & .61 & GRA & $A-C$ & .53 & .82 & $1.00 \bullet$ & .62 \\
\hline GRA & $A$ & .54 & .64 & .79 & .58 & HUM & O-c & .53 & .64 & .72 & .69 \\
\hline PER & $N$ & .52 & .57 & .70 & .67 & CAP & $A-C$ & .48 & .74 & .91 & .61 \\
\hline SOC & 0 & .49 & .58 & .73 & .59 & $\mathrm{CIT}$ & $A-C$ & .48 & .73 & .95 & .56 \\
\hline APP & 0 & .48 & .56 & .71 & .58 & SOC & $A-C$ & .47 & .72 & .91 & .62 \\
\hline CAP & $A$ & .45 & .54 & .66 & .53 & LOV & O-c & .46 & .56 & .68 & .62 \\
\hline CIT & $A$ & .45 & .53 & .69 & .52 & APP & $A-c$ & .42 & .65 & .82 & .61 \\
\hline SEL & $C$ & .43 & .48 & .66 & .47 & SEL & $C-p$ & .40 & .53 & .74 & .51 \\
\hline FOR & $A$ & .42 & .50 & .57 & .52 & JUD & C-r & .40 & .57 & .76 & .53 \\
\hline VAL & $E$ & .41 & .48 & .58 & .45 & FOR & $A-t$ & .40 & .75 & .86 & .53 \\
\hline JUD & C & .37 & .41 & .54 & .45 & MOD & $\mathrm{E}-\mathrm{a}$ & .36 & .45 & .59 & .57 \\
\hline PRU & C & .33 & .37 & .44 & .56 & VAL & E-a & .36 & .44 & .53 & .46 \\
\hline MOD & $E$ & .30 & .36 & .47 & .55 & PRU & C-o & .33 & .38 & .45 & .61 \\
\hline SPI & $A$ & .29 & .35 & .40 & .36 & SPI & $A-C$ & .32 & .49 & .57 & .37 \\
\hline Mean & & .52 & .60 & .72 & .60 & Mean & & .51 & .70 & .83 & .63 \\
\hline
\end{tabular}

Notes. $N=476 ;{ }^{a} N=475$. Scale = IPIP-VIA-R strength scale; $r=\max$. (uncorrected) absolute bivariate correlation with BFI-2-S domain or facet (in descending order); $r^{+}=$single disattenuation for unreliability in BFI-2-S domain (Cronbach's Alpha for six items) or BFI-2-S facet (Spearman-Brown-correction for two-item correlation; see Eisinga et al., 2013); $r^{\ddagger}=$ double disattenuation for unreliability in BFI-2-S and strength scale (using omega estimate for the latter). $R_{\text {adj }}=$ multiple-R (square root of $R^{2}{ }^{2 d j}$ ) when regressing strength on five BFI-2-S domains or $15 \mathrm{BFI}-2-\mathrm{S}$ facets); • overadjustment (estimates $>1$ were limited to 1.00 for computation of means). 


\section{Table 14}

IPIP-VIA-R: Gender Associations and Criterion Validity of Character Strengths (Samples 4 and 5)

\begin{tabular}{|c|c|c|c|c|c|c|}
\hline \multirow[b]{2}{*}{ Scale } & \multicolumn{2}{|c|}{ Gender } & \multicolumn{2}{|c|}{ Life Sat. } & \multicolumn{2}{|c|}{ Health } \\
\hline & UK & $\mathrm{DE}$ & UK & $\mathrm{DE}$ & UK & $\mathrm{DE}$ \\
\hline APP & .15 & .16 & .10 & .29 & .05 & .22 \\
\hline CAP & .11 & .13 & .38 & .42 & .16 & .22 \\
\hline CIT & .16 & .01 & .24 & .31 & .08 & .19 \\
\hline CUR & .05 & $.07^{a}$ & .32 & $.37^{a}$ & .14 & $.31^{\mathrm{a}}$ \\
\hline EQU & .15 & .12 & .15 & .22 & .02 & .07 \\
\hline FOR & .04 & -.07 & .29 & .27 & .09 & .17 \\
\hline GRA & .20 & .13 & .24 & .40 & .03 & .24 \\
\hline HOP & -.01 & -.07 & .45 & .46 & .14 & .24 \\
\hline HUM & .08 & -.04 & .29 & .34 & .11 & .22 \\
\hline IND & .05 & .02 & .35 & .40 & .16 & .18 \\
\hline INT & .15 & .13 & .13 & .13 & .02 & -.02 \\
\hline JUD & .00 & .04 & .15 & .16 & -.02 & .13 \\
\hline KIN & .19 & .14 & .19 & .25 & .03 & .13 \\
\hline LEA & -.01 & -.03 & .26 & .32 & .07 & .25 \\
\hline LOV & .12 & .02 & .15 & .30 & .05 & .27 \\
\hline MOD & .10 & .05 & -.12 & -.05 & -.10 & -.18 \\
\hline ORI & -.07 & .03 & .19 & .23 & .06 & .21 \\
\hline PER & .02 & .06 & .25 & .37 & .09 & .27 \\
\hline PRU & .05 & .18 & -.03 & -.05 & -.08 & -.13 \\
\hline SEL & -.12 & .02 & .19 & .14 & .13 & .13 \\
\hline SOC & .13 & .08 & .29 & .27 & .16 & .19 \\
\hline SPI & .16 & .20 & .35 & .22 & .03 & .12 \\
\hline VAL & -.09 & -.04 & .16 & .15 & .00 & .14 \\
\hline ZES & .03 & $-.02^{\mathrm{a}}$ & .54 & $.59^{\mathrm{a}}$ & .33 & $.36^{\mathrm{a}}$ \\
\hline Mean & .07 & .06 & .23 & .27 & .07 & .17 \\
\hline$N$ & 474 & 476 & 474 & 476 & 474 & 476 \\
\hline
\end{tabular}

Notes. ${ }^{a} N=475$, UK = United Kingdom, DE = Germany; Scale = IPIP-VIA-R strength, Life Sat. = Life Satisfaction; for all coefficients, $p<.05$ if $|r|>.09 ; p<.01$ if $|r|>.12, p<.001$ if $|r|>.16$; bold font type: $r \geq .20$ (at least medium effect size). 
Table 15

IPIP-VIA-R: Incremental Criterion Validity of Character Strengths beyond Big Five Domains (Samples 4 and 5)

\begin{tabular}{|c|c|c|c|c|c|c|c|c|c|c|c|c|}
\hline \multirow{4}{*}{$\begin{array}{l}\text { M0: BFI } \\
\text { M1: +Scale }\end{array}$} & \multicolumn{6}{|c|}{ Life Satisfaction } & \multicolumn{6}{|c|}{ Health } \\
\hline & \multirow{2}{*}{\multicolumn{3}{|c|}{$\begin{array}{c}\text { UK }(N=474) \\
R_{\text {adj }}^{2}=.31(p<.001)\end{array}$}} & \multirow{2}{*}{\multicolumn{3}{|c|}{$\begin{array}{c}\mathrm{DE}\left(N=476^{\mathrm{a}}\right) \\
2_{\text {adj }}=.32(p<.001)\end{array}$}} & \multirow{2}{*}{\multicolumn{3}{|c|}{$\begin{array}{c}\text { UK }(N=474) \\
{ }_{\text {adj }}=.16(p<.001)\end{array}$}} & \multirow{2}{*}{\multicolumn{3}{|c|}{$\begin{array}{c}\mathrm{DE}\left(N=476^{\mathrm{a}}\right) \\
R_{\text {adj }}^{2}=.14(p<.001)\end{array}$}} \\
\hline & & & & & & & & & & & & \\
\hline & $r^{2}$ & $\Delta R^{2}{ }_{\text {adj }}$ & $\beta$ & $r^{2}$ & $\Delta R^{2}{ }_{\text {adj }}$ & $\beta$ & $r^{2}$ & $\Delta R^{2}$ adj & $\beta$ & $r^{2}$ & $\Delta R^{2}{ }_{\text {adj }}$ & $\beta$ \\
\hline APP & .01 & .00 & -.03 & .08 & .01 & .15 & .00 & .00 & .03 & .05 & .01 & .14 \\
\hline CAP & .15 & .02 & .20 & .17 & .04 & .24 & .03 & .00 & .05 & .05 & .01 & .14 \\
\hline CIT & .06 & .00 & -.04 & .10 & .01 & .10 & .01 & .00 & -.10 & .04 & .01 & .10 \\
\hline CUR & .10 & .00 & .07 & .14 & .01 & .14 & .02 & .00 & -.03 & .10 & .02 & .18 \\
\hline EQU & .02 & .00 & -.06 & .05 & .00 & .06 & .00 & .00 & -.01 & .01 & .00 & .02 \\
\hline FOR & .09 & .00 & .07 & .07 & .00 & .06 & .01 & .00 & .00 & .03 & .00 & .08 \\
\hline GRA & .06 & .00 & .05 & .16 & .06 & .29 & .00 & .00 & -.04 & .06 & .03 & .21 \\
\hline HOP & .21 & .01 & .16 & .21 & .00 & .11 & .02 & .01 & -.13 & .06 & .00 & -.04 \\
\hline HUM & .08 & .00 & .03 & .12 & .00 & .07 & .01 & .00 & -.05 & .05 & .00 & .02 \\
\hline IND & .12 & .01 & .15 & .16 & .01 & .13 & .02 & .00 & .02 & .03 & .00 & .03 \\
\hline INT & .02 & .01 & -.12 & .02 & .00 & -.08 & .00 & .00 & -.06 & .00 & .01 & -.12 \\
\hline JUD & .02 & .00 & -.06 & .02 & .00 & .01 & .00 & .01 & -.15 & .02 & .00 & .05 \\
\hline KIN & .04 & .00 & -.05 & .06 & .00 & .01 & .00 & .00 & -.06 & .02 & .00 & .02 \\
\hline LEA & .07 & .00 & -.10 & .10 & .00 & .01 & .01 & .04 & -.29 & .06 & .00 & .04 \\
\hline LOV & .02 & .00 & .04 & .09 & .01 & .11 & .00 & .00 & .00 & .07 & .01 & .14 \\
\hline MOD & .01 & .02 & -.17 & .00 & .00 & -.08 & .01 & .00 & -.02 & .03 & .01 & -.14 \\
\hline ORI & .04 & .00 & -.03 & .05 & .00 & .00 & .00 & .01 & -.15 & .05 & .00 & .00 \\
\hline PER & .06 & .00 & -.05 & .13 & .00 & .07 & .01 & .00 & -.10 & .07 & .00 & .11 \\
\hline PRU & .00 & .00 & -.03 & .00 & .00 & .03 & .01 & .00 & -.02 & .02 & .00 & -.01 \\
\hline SEL & .04 & .00 & -.09 & .02 & .01 & -.11 & .02 & .00 & .00 & .02 & .00 & .05 \\
\hline SOC & .08 & .00 & -.03 & .07 & .00 & .04 & .03 & .00 & -.05 & .04 & .00 & .03 \\
\hline SPI & .12 & .03 & .19 & .05 & .01 & .12 & .00 & .00 & -.06 & .02 & .00 & .06 \\
\hline VAL & .03 & .00 & -.02 & .02 & .00 & -.07 & .00 & .02 & -.17 & .02 & .00 & -.01 \\
\hline ZES & .29 & .05 & .32 & .35 & .07 & .40 & .11 & .02 & .20 & .13 & .03 & .29 \\
\hline
\end{tabular}

Notes. ${ }^{\mathrm{a}} \mathrm{N}=475$ for CUR and ZES; UK = United Kingdom, DE = Germany, Scale = IPIP-VIA-R strength; M0: Baseline model for each criterion and each country only comprising the scale scores of the five Big Five domains as predictors, $R^{2}$ adj $=$ multiple $R$-squared (adjusted) of each baseline model; M1: Model additionally including scale score of IPIP-VIA-R strength as predictor, $r^{2}=$ squared bivariate correlation of IPIP-VIA-R strength with criterion, $\Delta R^{2}$ adj $=$ difference in multiple- $R$ squared (adjusted) between $\mathrm{M} 0$ and $\mathrm{M} 1, \beta=$ standardized regression coefficient of IPIP-VIA-R strength; bold font type: $p<.05$. 
HUMAN CHARACTER IN THE IPIP

\section{Figures}



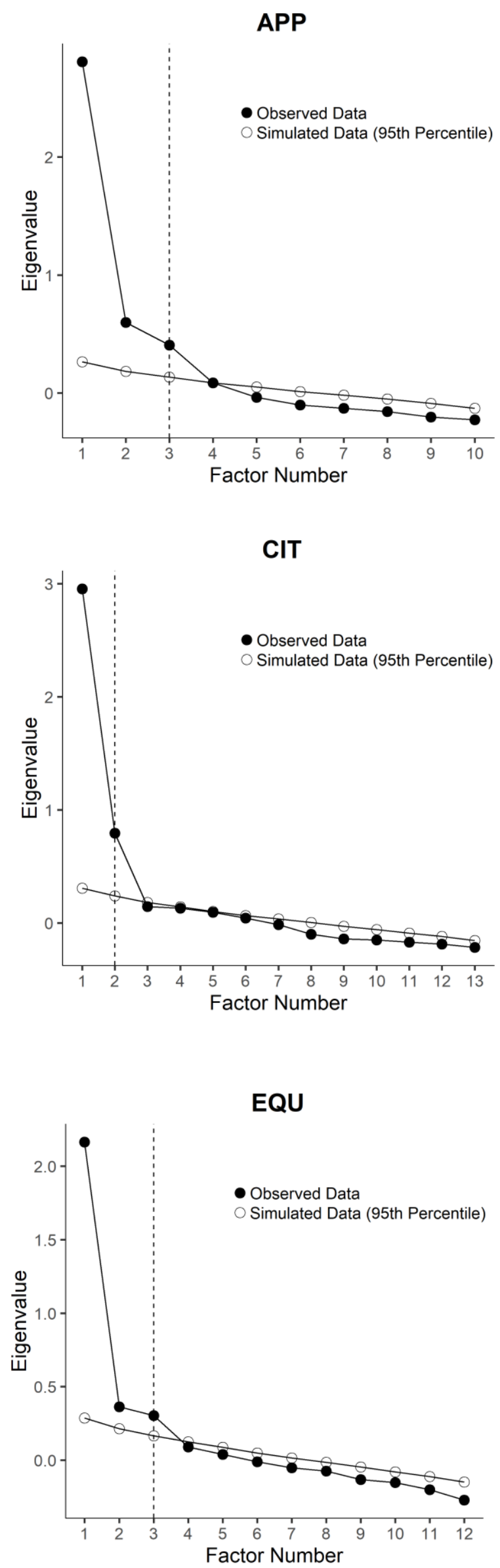

CAP

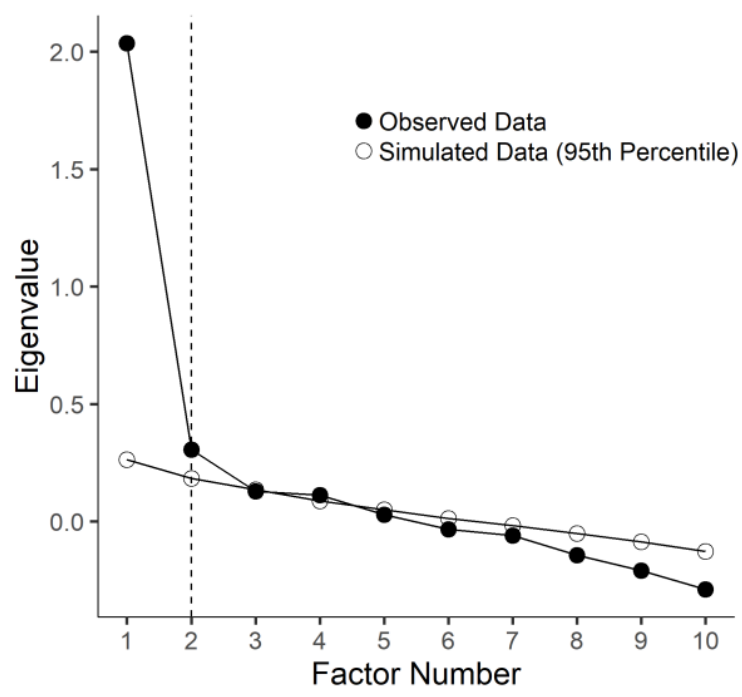

CUR

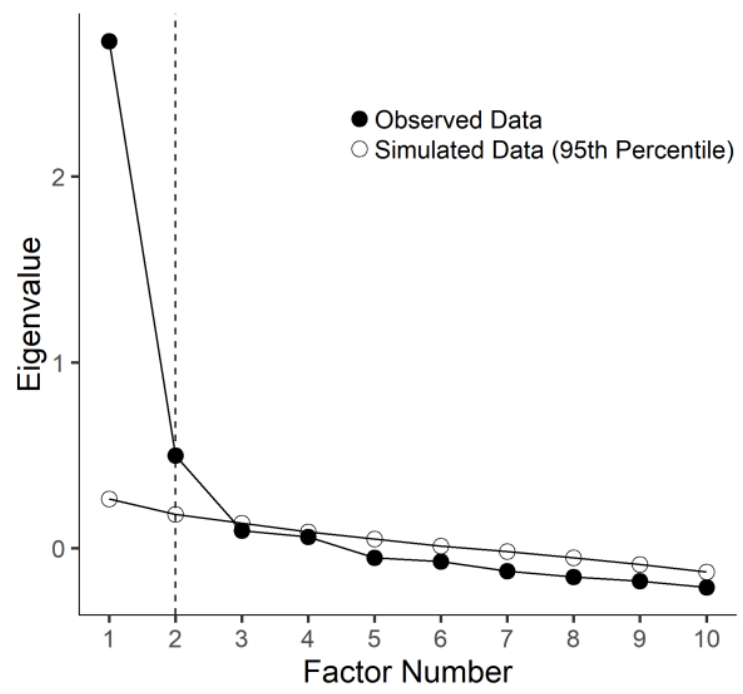

FOR

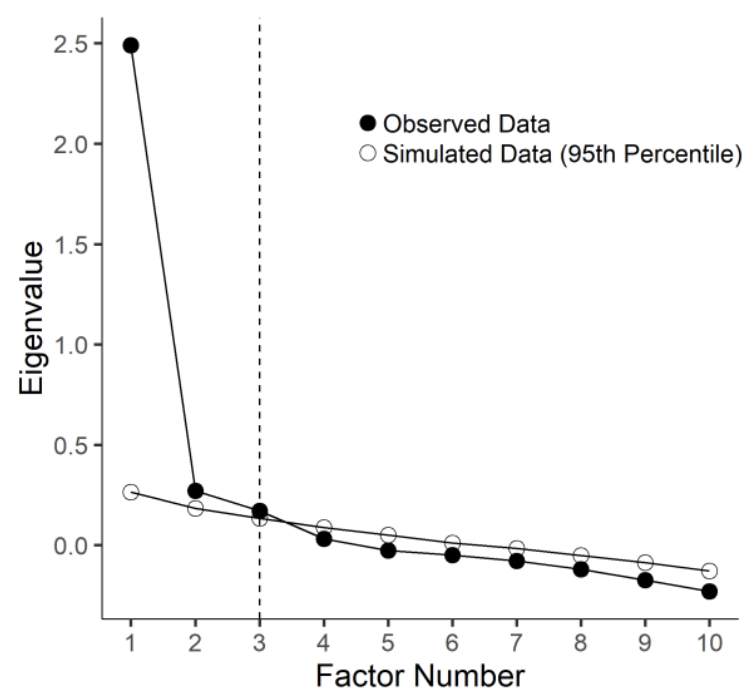


GRA

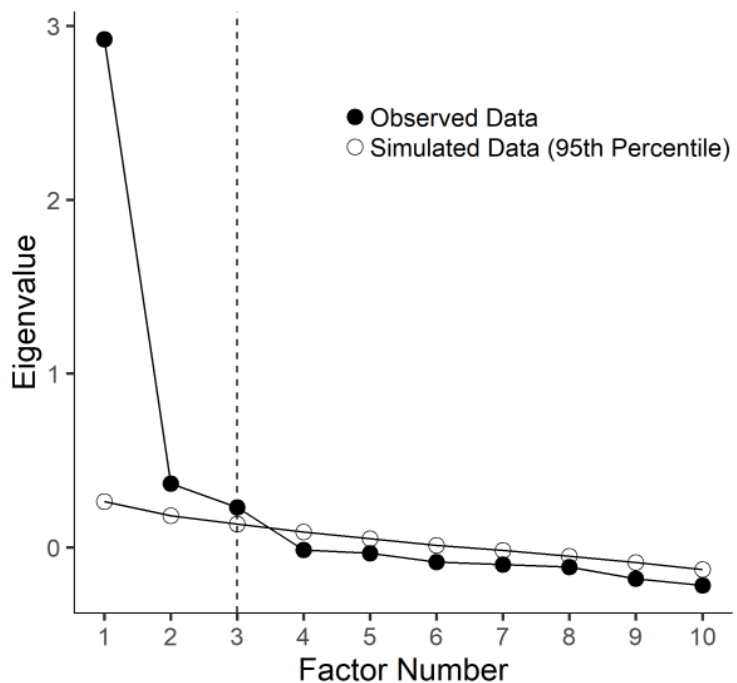

HUM

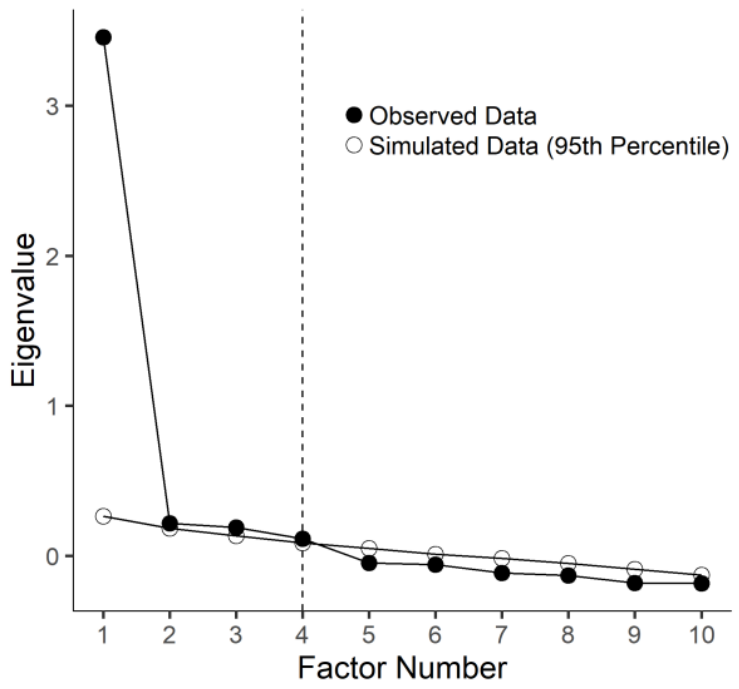

INT

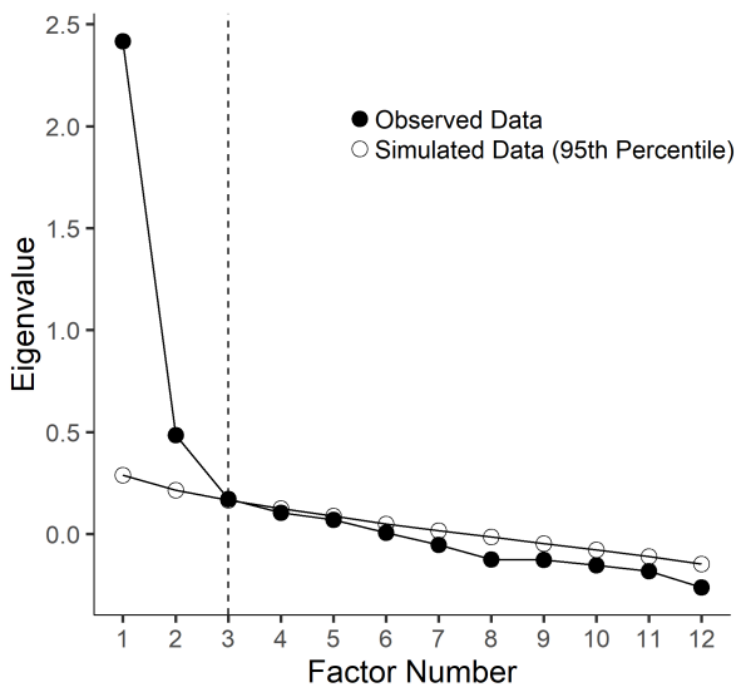

HOP

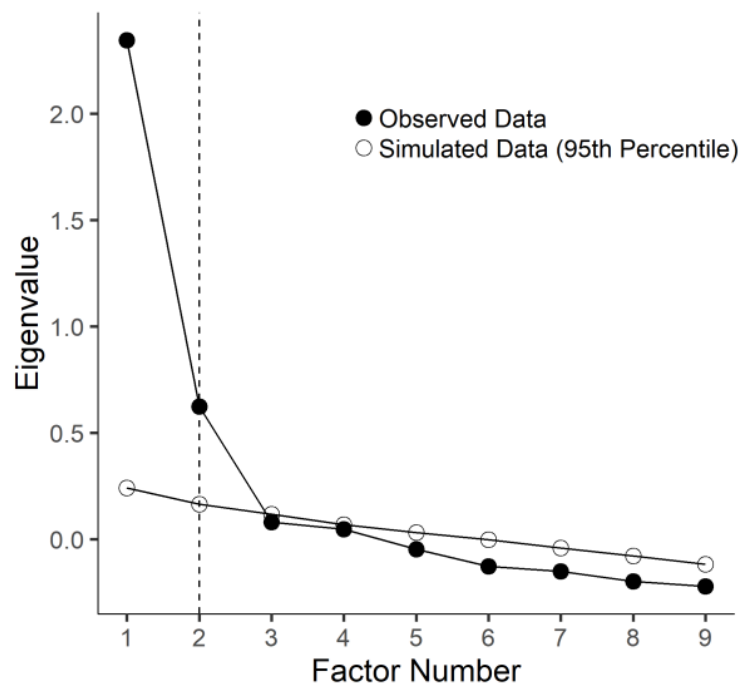

IND

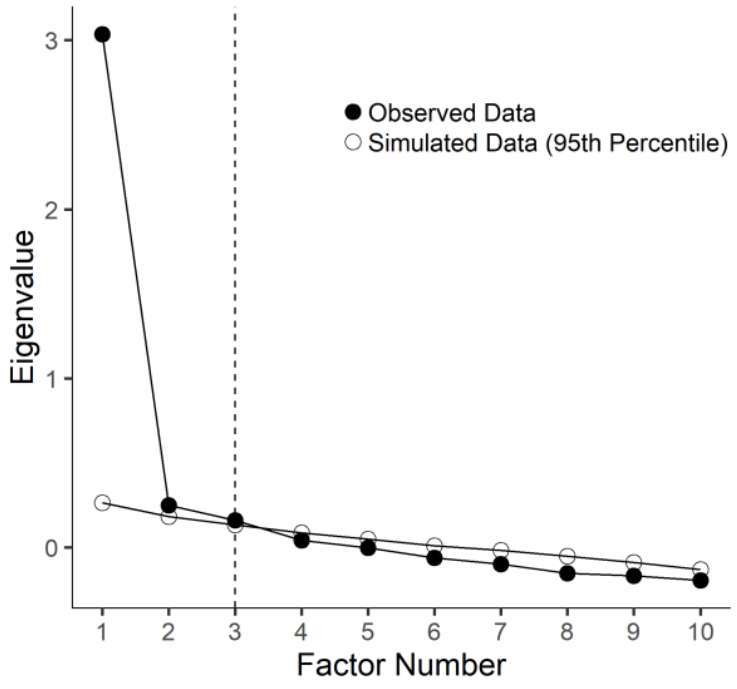

JUD

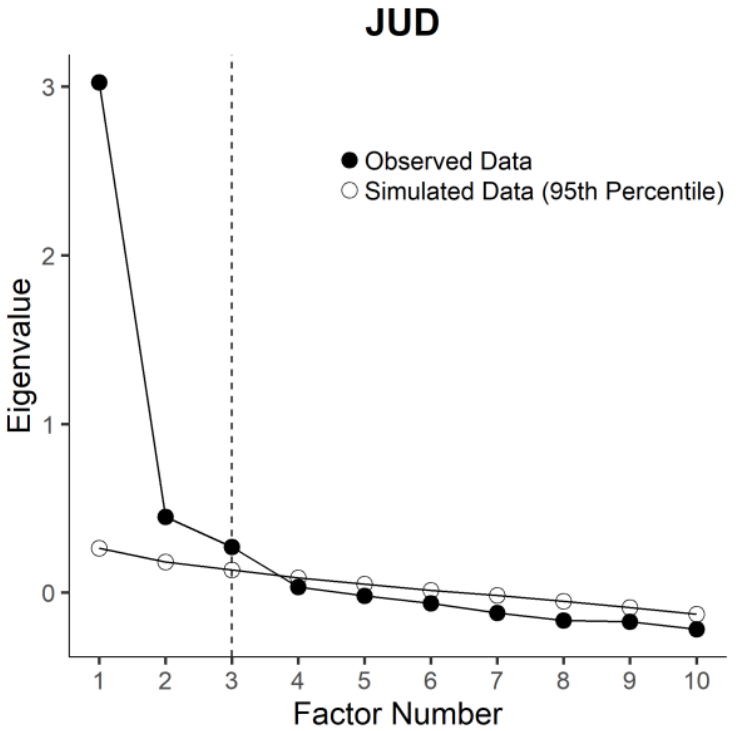


KIN

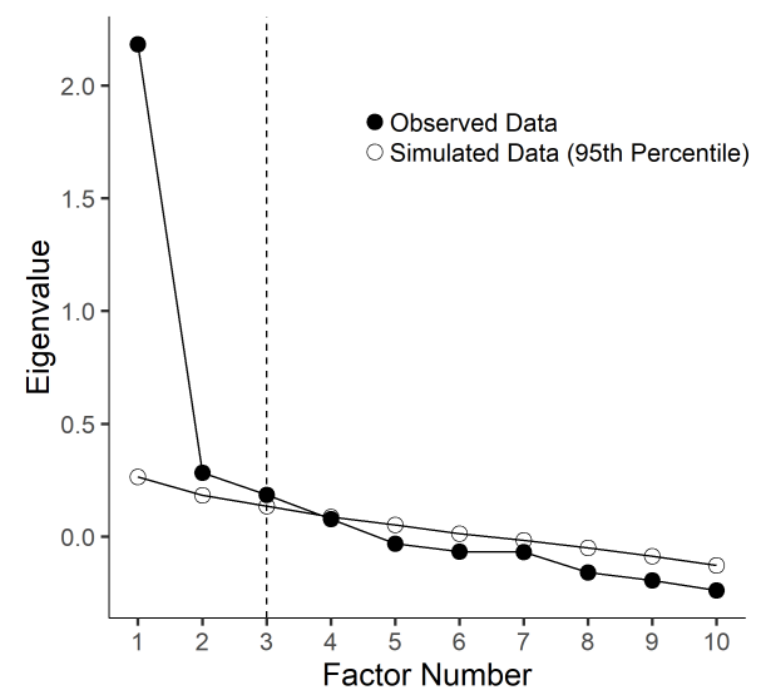

LOV

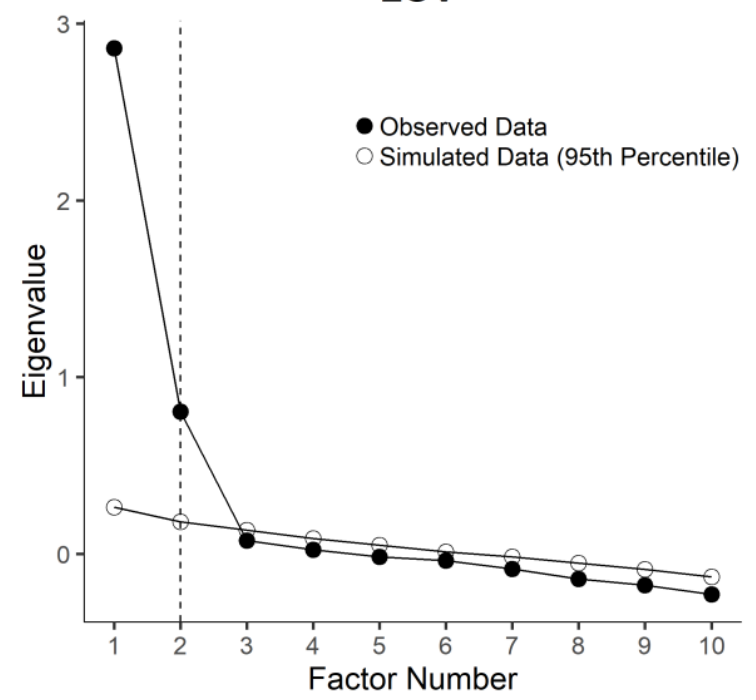

ORI

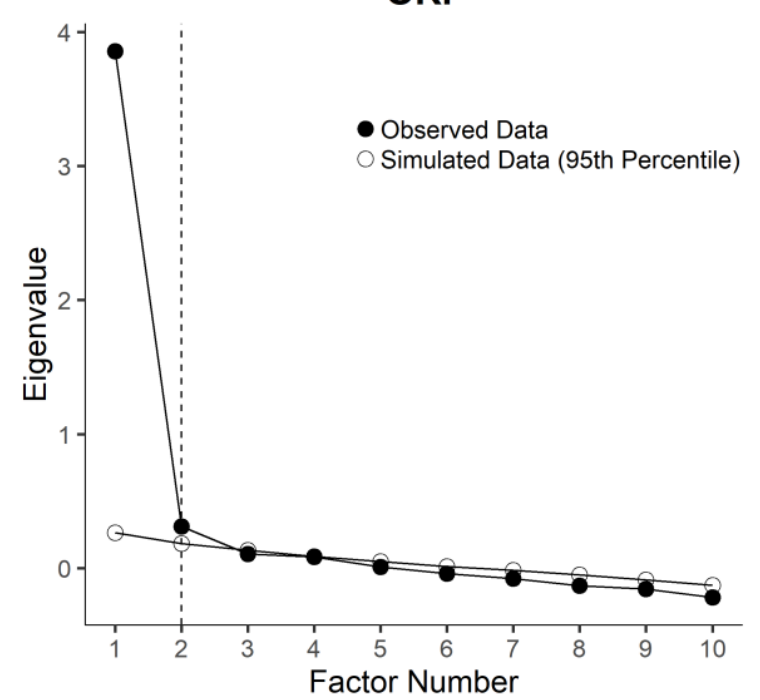

LEA

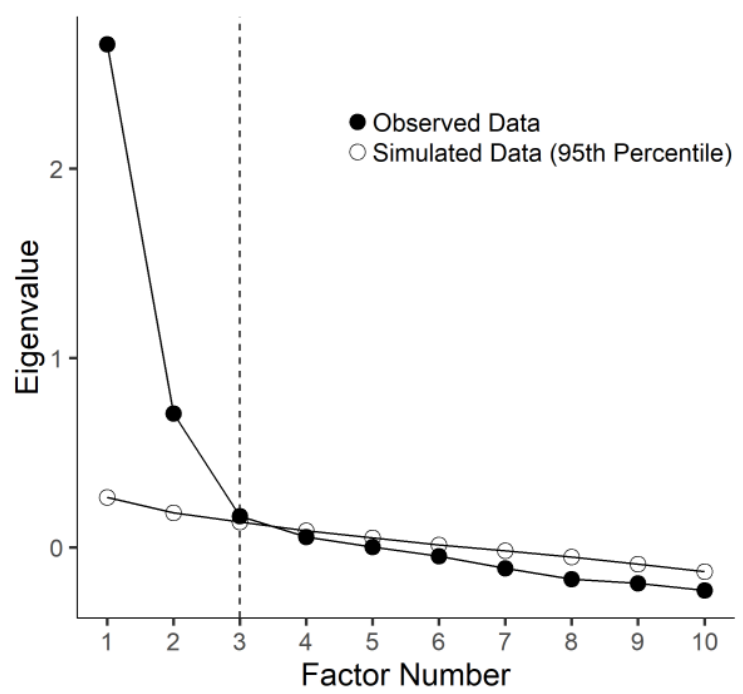

MOD

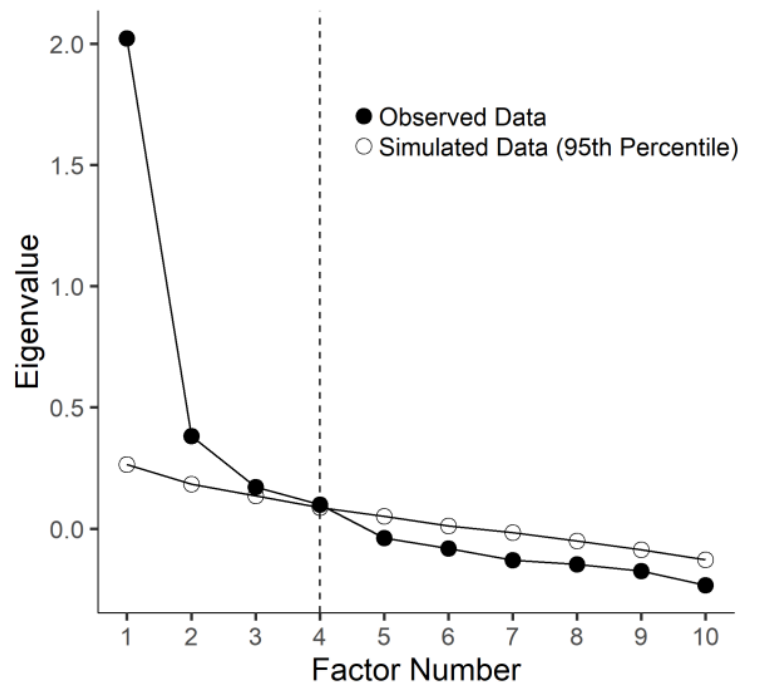

PER

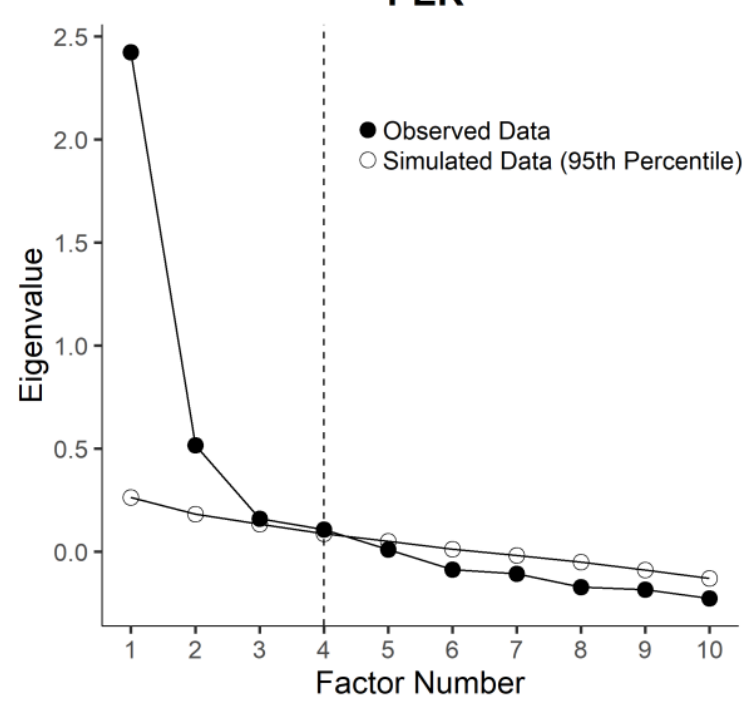


PRU

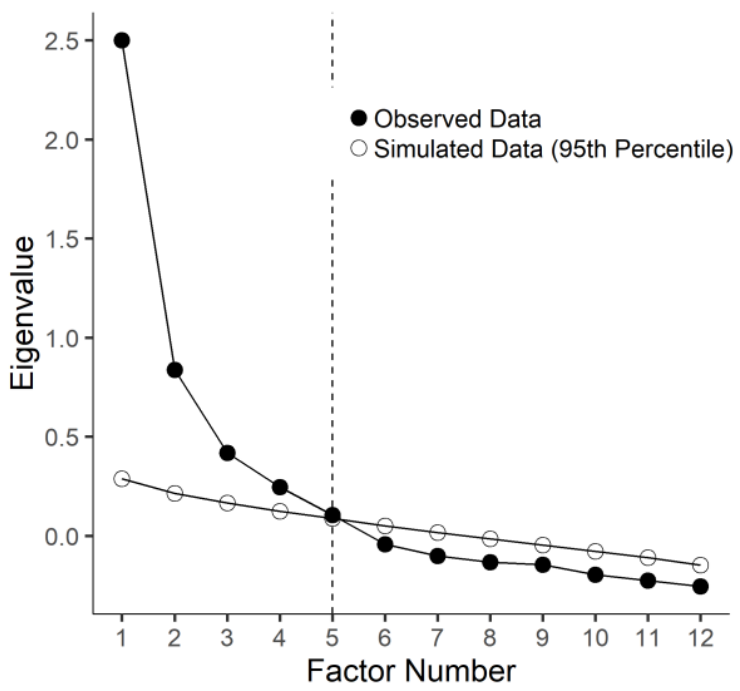

SOC

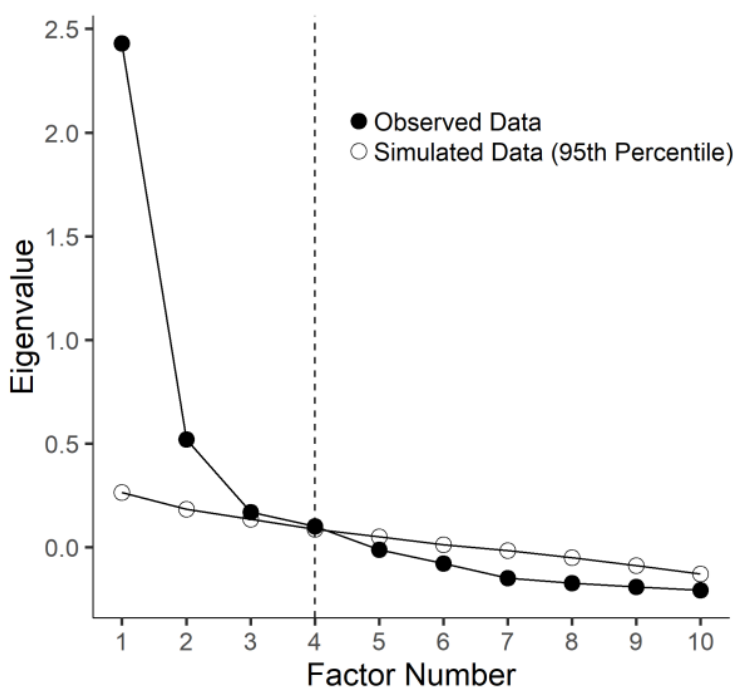

VAL

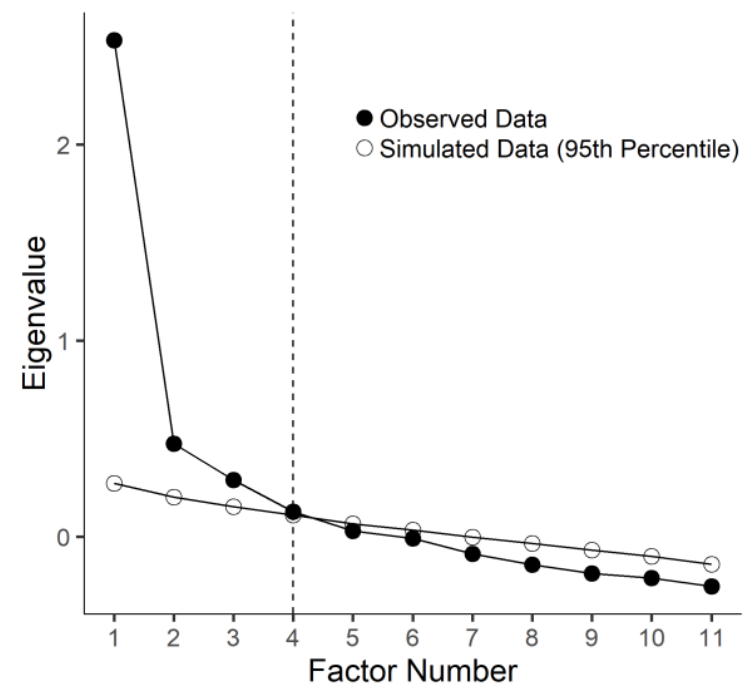

SEL

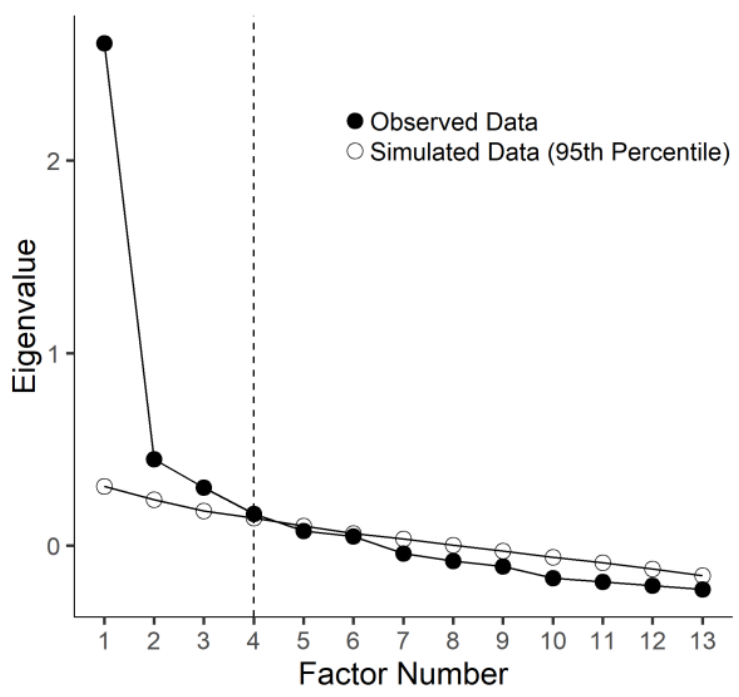

SPI

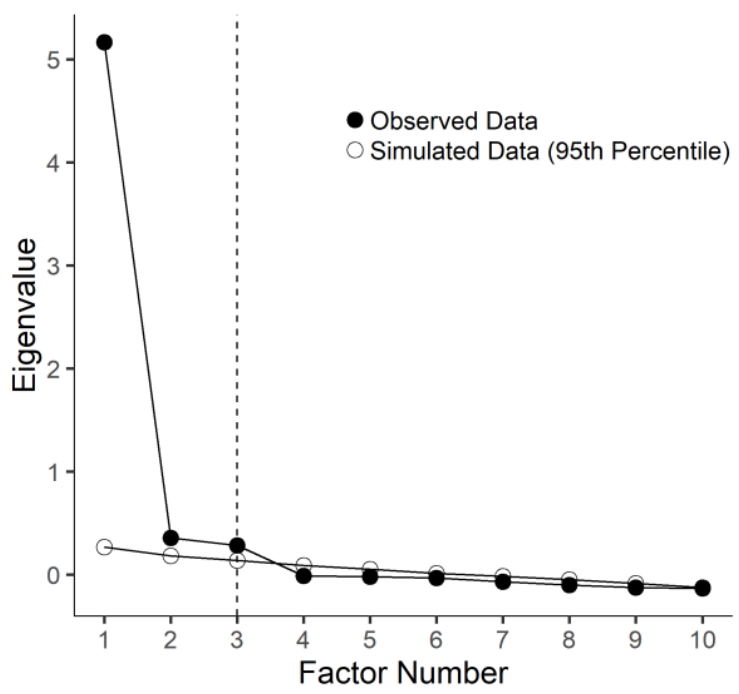

ZES

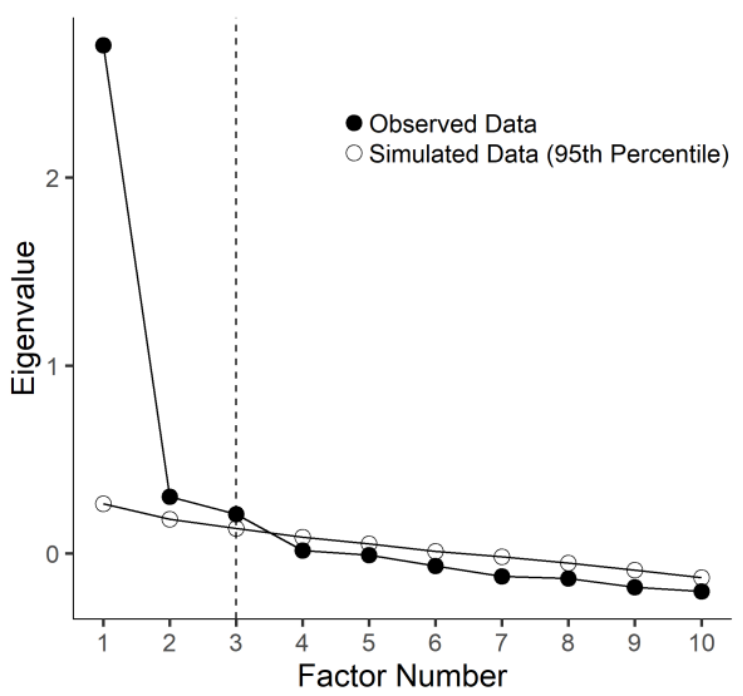

Figure 1: Scree-plots from 24 principal axis factor analyses of IPIP-VIA strengths with number of factors as indicated for each by parallel analyses (Sample 1: ESCS). 


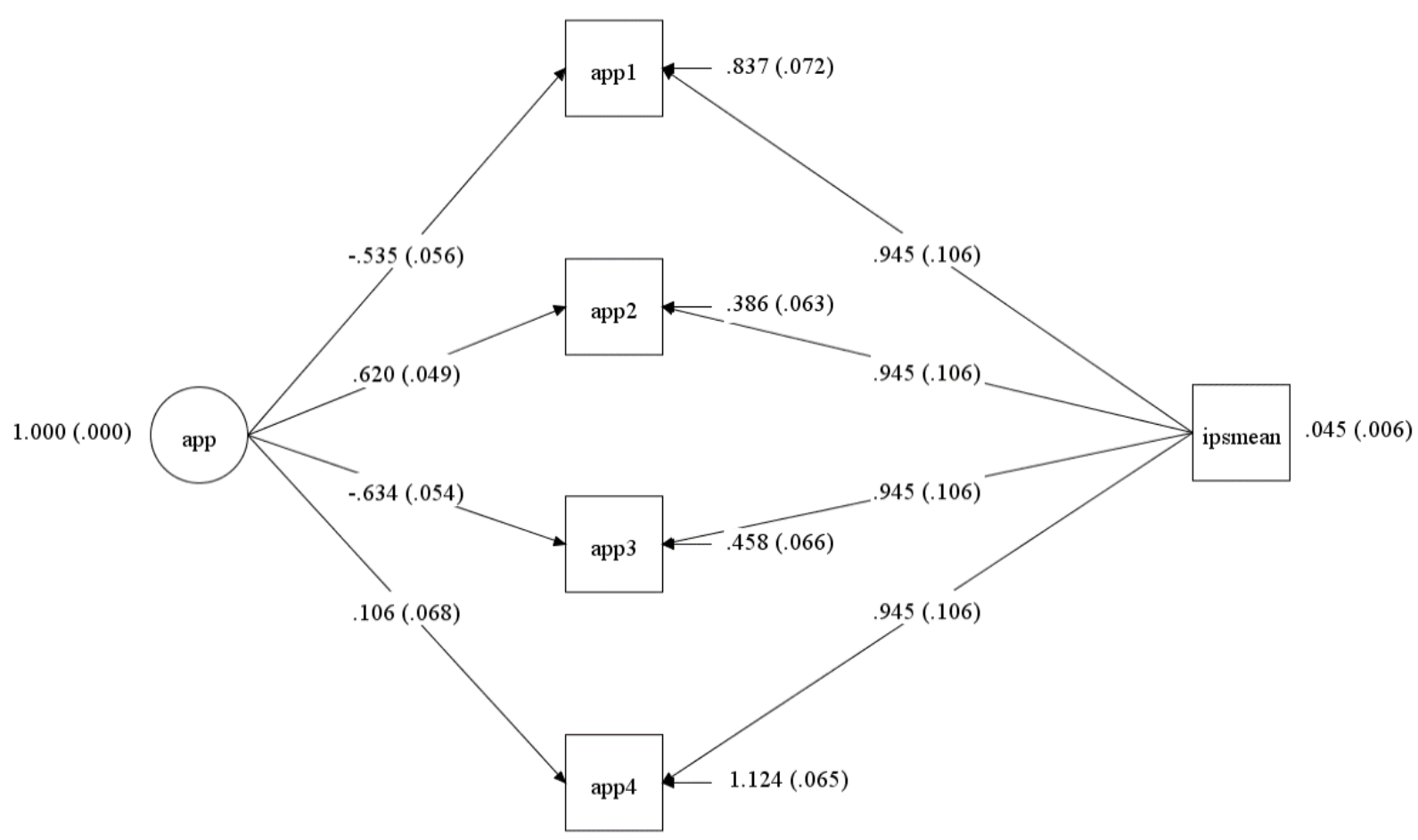

Figure 2: CFA measurement model for a common latent variable (here: Appreciation of Beauty and Excellence, variant A, depicted as 'app') based on four indicators (app1-app4). Acquiescence is controlled by a manifest covariate 'ipsmean' (i.e., the ipsative mean across 96 VIA items), which impacts on all indicators with the same weight irrespective of keying direction. 


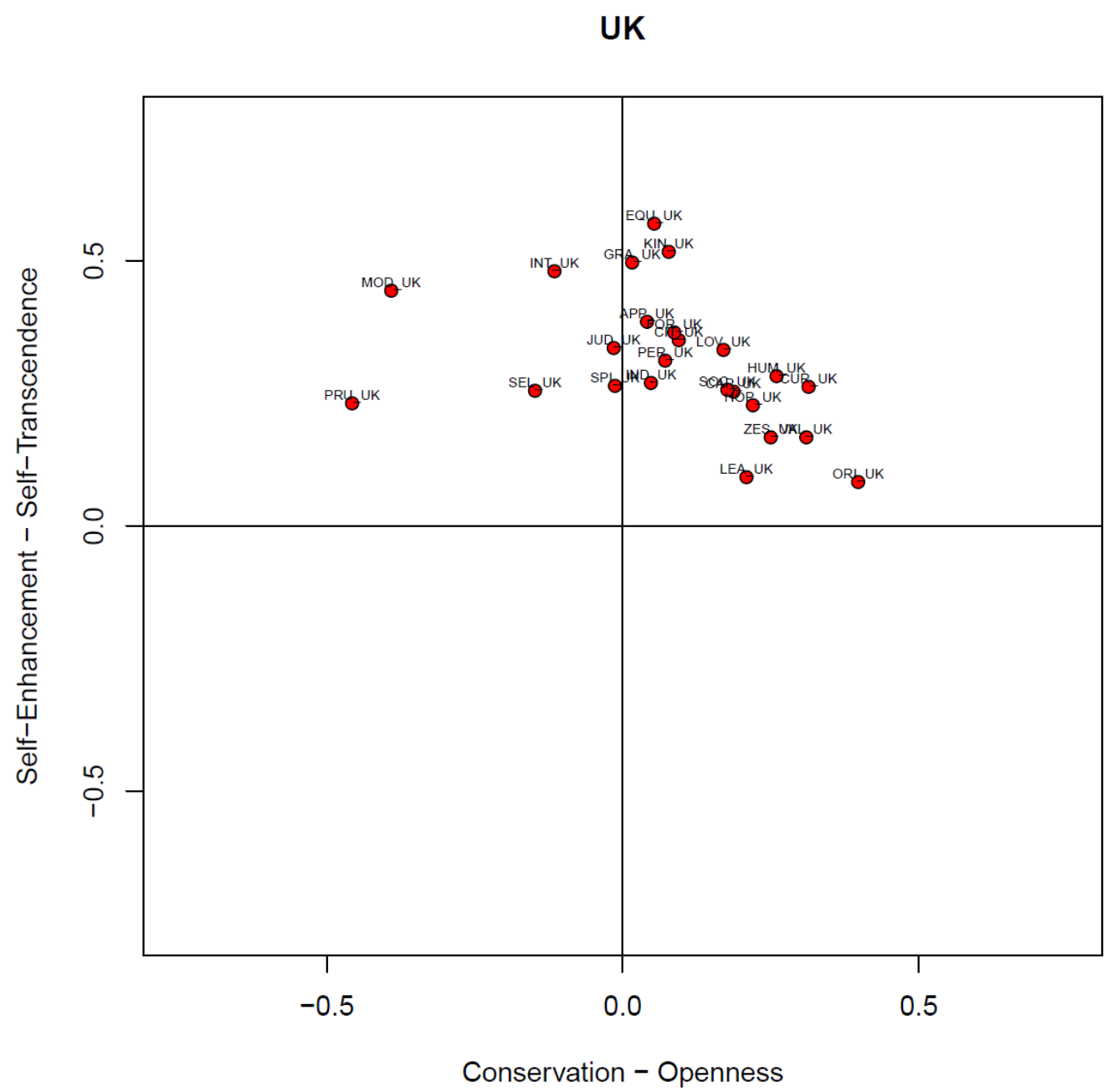

Figure 3a: Plot for 24 IPIP-VIA-R scales in the higher-order dimensional space of Schwartz human values in the UK (United Kingdom). 


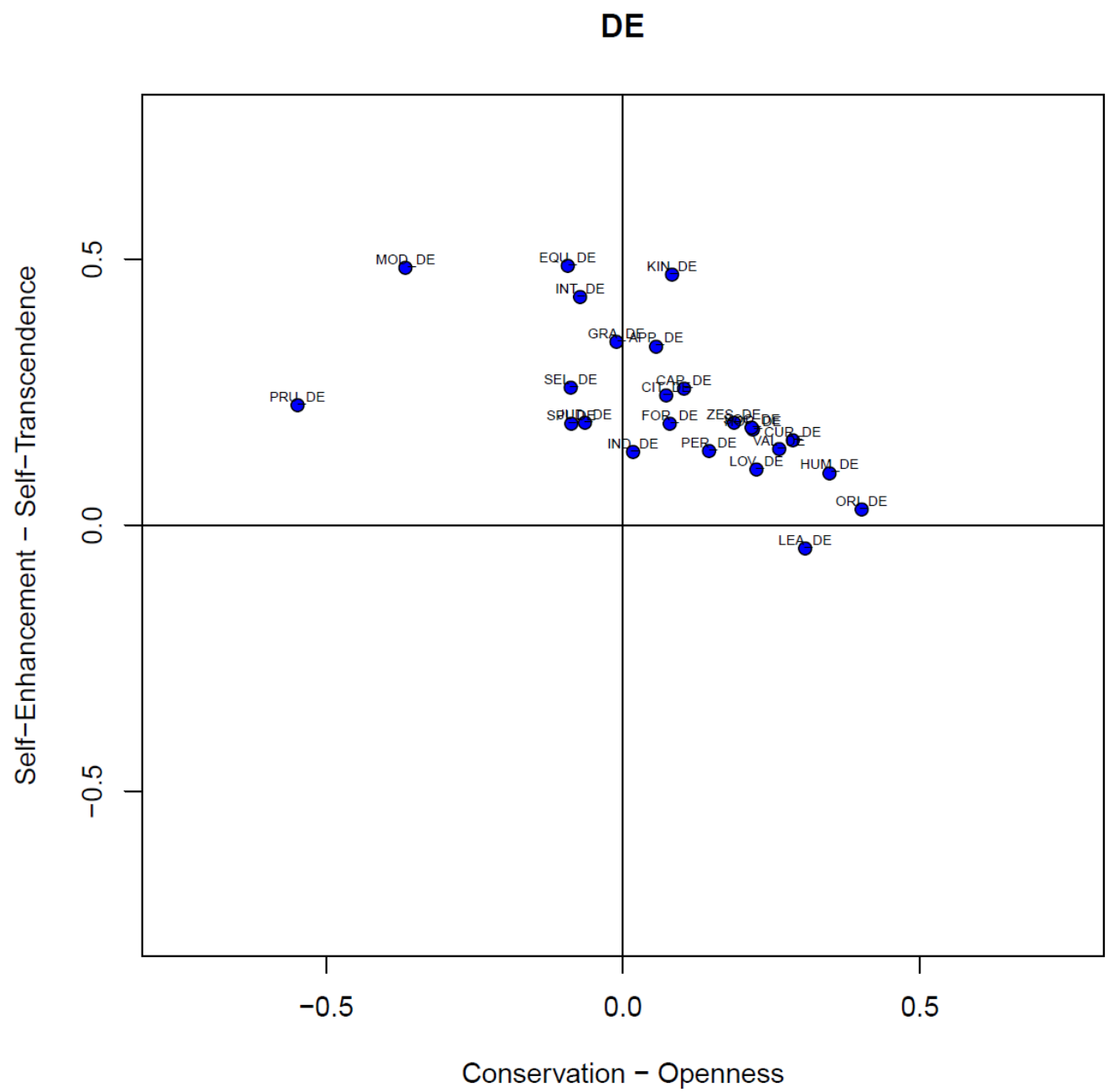

Figure 3b: Plot for 24 IPIP-VIA-R scales in the higher-order dimensional space of Schwartz human values in DE (Germany). 


\section{UK \& DE}

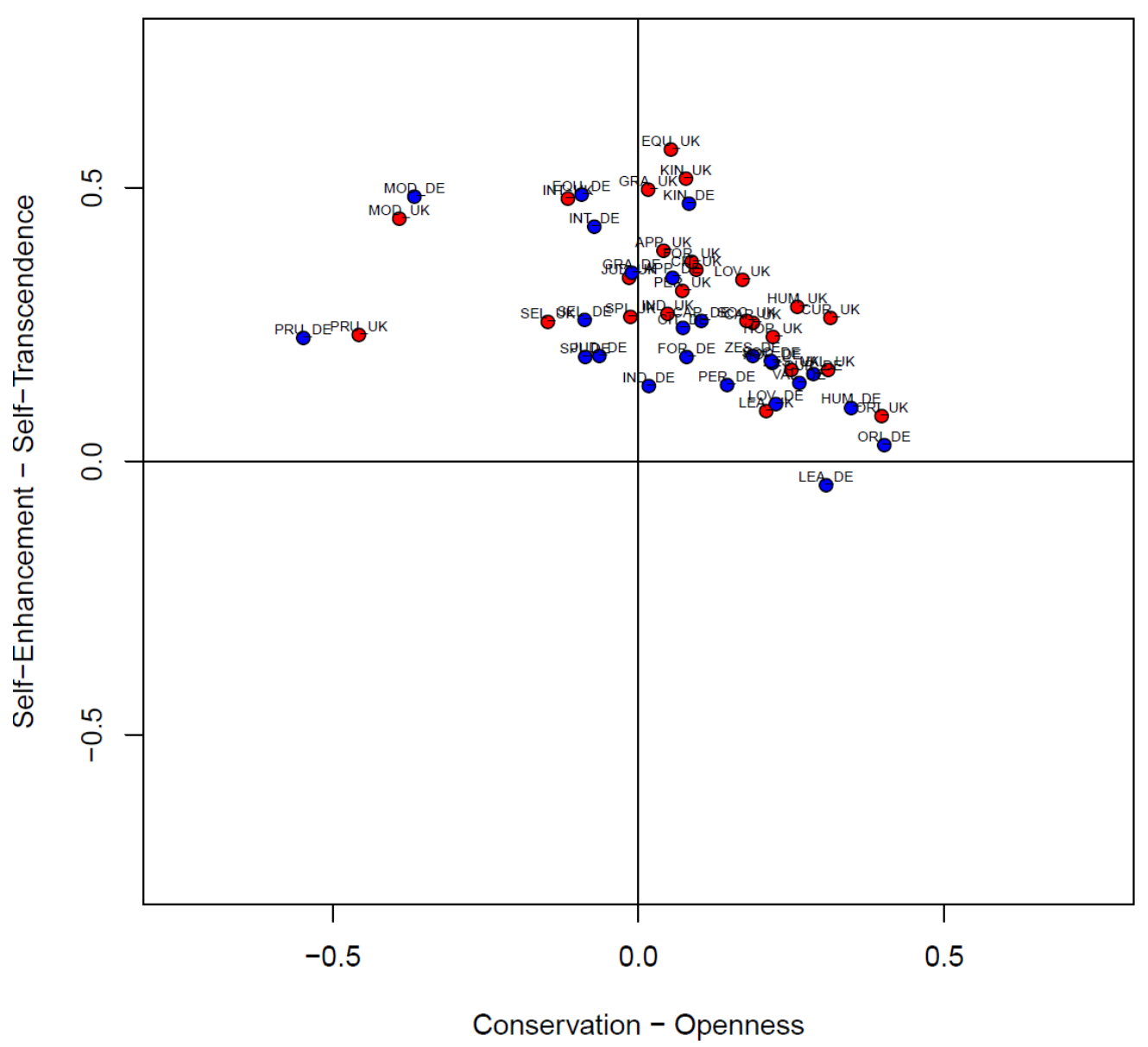

Figure 3c: Congruence plot for 24 IPIP-VIA-R scales in the higher-order dimensional space of Schwartz human values across the United Kingdom (UK, red dots) and Germany (DE, blue dots). 
UK \& DE: Eucl. Distances

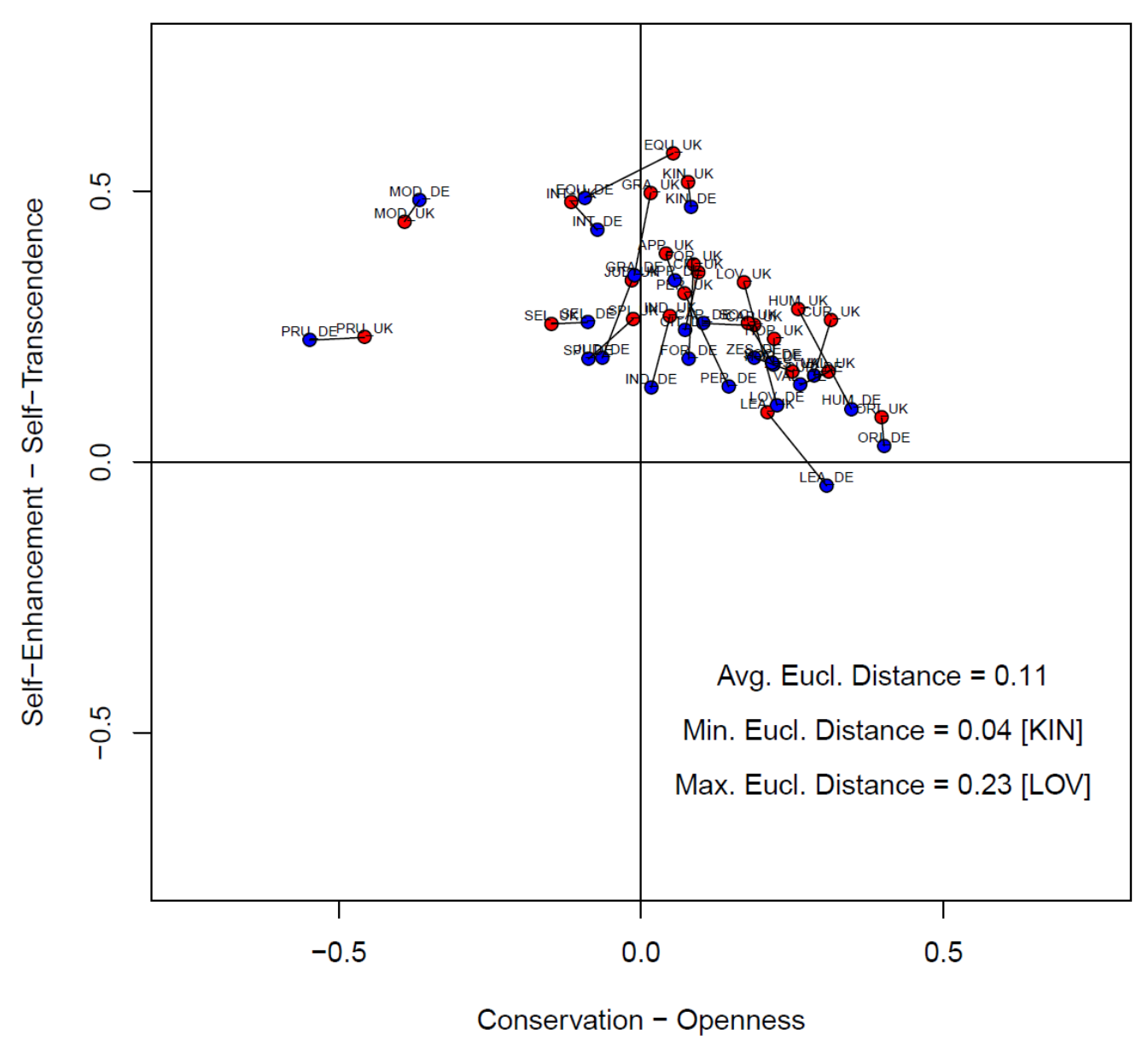

Figure 3d: Euclidean distances in the congruence plot for 24 IPIP-VIA-R scales in the higher-order dimensional space of Schwartz human values across the United Kingdom (UK, red dots) and Germany (DE, blue dots). Cross-country differences are indicated by solid lines. 